This document is downloaded from DR-NTU (https://dr.ntu.edu.sg) Nanyang Technological University, Singapore.

Soft-error tolerant design for satellite-board computations

Zhang, Lei

2016

https://hdl.handle.net/10356/69206

https://doi.org/10.32657/10356/69206 


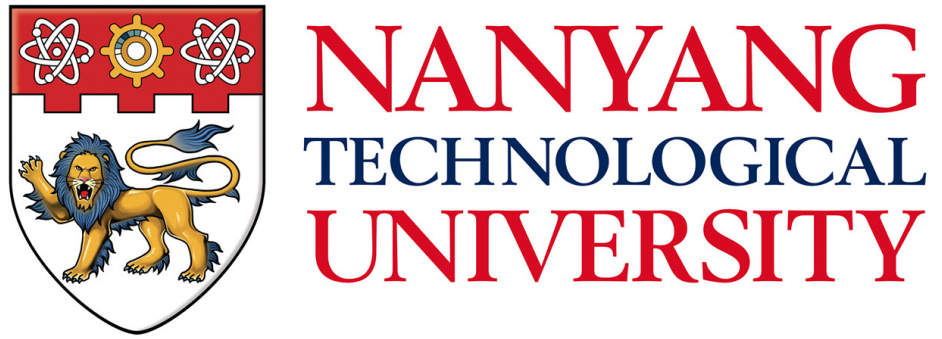

\title{
Soft-Error Tolerant Design for Satellite-Board Computations
}

\author{
ZHANG Lei \\ Thesis Supervisor: \\ Assoc. Prof. Hsu Wen-Jing \\ School of Computer Science and Engineering \\ A thesis submitted to the Nanyang Technological University \\ in partial fulfilment of the requirement for the degree of \\ Master of Engineering
}





\section{Abstract}

High energy particles in the outer space could flip the state of the latches of the electronic devices. The upset of the latches is called soft error since it would not cause permanent damage to the device. However, the soft error may cause faults in a processor and lead to malfunction of the computer system on the satellite.

In this thesis, we implement a novel soft error fault tolerant scheme based on the LEON2/3 processor to protect the processor from soft errors. We verify the correctness and evaluate the overhead of this scheme, and we also determine the critical resource which should be protected.

Our scheme includes two parts: sensor network and rollback scheme. A sensor is used to monitor a target register. It will assert if the monitored register is flipped because of a soft error.

The rollback scheme is modified from a synchronization feature of the LEON2 and LEON3 processors. This feature originally aims to synchronize among Floating Point Unit (FPU), CACHE and Integer Unit (IU). We make use of it to stall the IU when a soft error is detected, and recover from the error by re-executing the current operation.

To verify the correctness of the sensor and rollback scheme, we inject errors during the execution of a large number of instructions of LEON2/3. The results show that all instruction rollbacks are correct.

To evaluate the overhead of the scheme, we determine the time and resource penalties of our scheme. The test results show that the scheme incurs only one extra clock penalty in about $90 \%$ of test cases and increases $0.282 \%$ of resource usage of the original processor for adding one 32-bit sensor.

To identify the critical resource for protection, we define the weight of instructions based on the frequency of instruction usage. Moreover, we monitor the number of accesses of the internal registers/bits in LEON3. Then we compute the impact factor (IF) for each internal register and status bit according to the register access frequencies and instruction weight. Using this approach, we could figure out the most critical resources according to the impact factor. The results show that there are 233 registers and status bits in total. Of which, 
91 of them have $100 \%$ IF which suggests they are the critical resources. 43 of them have an IF ranging between $1.9 \%$ and $97.6 \%$, which means they are less important. The IF of the remaining 89 is 0 because they are not used in our target application.

Our work shows that the sensor network and rollback scheme can protect the processor from soft errors while incurring minimal penalties. By analyzing the vulnerability of registers and status bits, we can selectively deploy limited resource on the most critical registers/bits. Therefore, our scheme could effectively improve the robustness of computer systems for satellite applications. 


\section{Acknowledgments}

I would like to express my great gratitude to my supervisor, Prof. Hsu Wen Jing, for his patient and invaluable guidance throughout the project and my thesis writing. Prof. Hsu deeply impressed me with his meticulous attitude towards research and working and showed me a great model of a researcher. His spirit encouraged me during my study and will continue to guide me in the future.

I would like to express my special gratitude to Prof. Zhang Wei and Dr. Teo Kian Jin Jason for providing me a valuable opportunity to work and study in NTU. Dr. Teo was not only a great teacher to guide me in the research but also a good friend to care about my personal life to give me his valuable advice.

I would also like to thank Prof. Lam Siew Kei and Dr. Cui Yingnan for giving me ideas and help in my research. Dr. Cui also taught me on how to write a legitimate thesis and helped me to improve my thesis. I appreciate his generous help.

I am grateful to my seniors and friends and would like to thank, Yang Liwei, Dr. Jiang Lianlian, Mao Fubing, Dr. Zhou Bin, Xu Dongjun, Nitin Shivaraman and Sriram Vasudevan, for their various kinds of help and sharing of their experience.

Finally and most of all, I would like to thank my parents for their continued support. Although they were far away from me, their love, understanding and motivation had been encouraging me to fight for a promising future.

Zhang Lei 



\section{Contents}

Abstract ..................... i

Acknowledgments ................... iii

1 Introduction 1

1.1 Computer System for Satellites . . . . . . . . . . . . . . 1

1.2 Soft Errors . . . . . . . . . . . . . . . . . . . . . . . 1

1.3 Previous Solutions . . . . . . . . . . . . . . . . 2

1.4 Objectives and Approach . . . . . . . . . . . . . . . . 3

1.5 Thesis Organization . . . . . . . . . . . . . . . . . 4

2 Literature Review $\quad 7$

2.1 Radiation Effects on Electronic Devices . . . . . . . . . . . . 7

2.1.1 High Energy Particles . . . . . . . . . . . . . . 7

2.1.2 Total Ionizing Dosage Effects . . . . . . . . . . . . 8

2.1.3 Single Event Effects . . . . . . . . . . . . . . . . 8

2.2 Solutions for Overcoming Radiation Effects . . . . . . . . . . . . . 11

2.2.1 Overcoming Total Ionizing Dosage Effects . . . . . . . . . 11

2.2.2 Overcoming Single Event Effects . . . . . . . . . . . . 12

2.3 Summary . . . . . . . . . . . . . . . . . 16

3 Design of Fault Tolerant Scheme 19

3.1 Design of Sensors Network . . . . . . . . . . . . . . . . . 19

3.1.1 Design of A Sensor . . . . . . . . . . . . . . . . . 19

3.1 .2 Sensors Network . . . . . . . . . . . . . . . 23

3.2 Implementation of Error Recovery Scheme . . . . . . . . . . . . . 24 
3.2 .1 Target Processor . . . . . . . . . . . . . . . . 24

3.2.2 Implementation on LEON2 . . . . . . . . . . . . . 25

3.2 .3 Migration on LEON3 . . . . . . . . . . . . . . . . . . 28

3.3 Chapter Summary . . . . . . . . . . . . . . . . . 31

4 Verification 33

4.1 System Design . . . . . . . . . . . . . . . . . . . . . . . 33

4.2 Verification on LEON2 . . . . . . . . . . . . . . . . . . . . 34

4.2 .1 Tool Chain . . . . . . . . . . . . . . . . 34

4.2 .2 Verification Method . . . . . . . . . . . . . . 35

4.2 .3 Test Automation . . . . . . . . . . . . . . . . . . . 38

4.2.4 Verification Results . . . . . . . . . . . . . . . . . . 39

4.3 Verification on LEON3 . . . . . . . . . . . . . . . . . . . . 41

4.3.1 Verification Method . . . . . . . . . . . . . . . . 41

4.3 .2 Verification Results . . . . . . . . . . . . . . . 42

4.4 Chapter Summary . . . . . . . . . . . . . . . . . . . . . . 43

5 Overhead Evaluation $\quad 45$

5.1 Performance Penalty . . . . . . . . . . . . . . . 45

5.1.1 The Measurement Method of Performance Overhead . . . 46

5.1 .2 Measurement Flow . . . . . . . . . . . . . . . . . . . . . . 48

5.1.3 Overall Setups for Performance Overhead Measurements . 50

5.1.4 Results of Performance Penalty Measurement . . . . . . . 53

5.2 Resource Overhead Measurement . . . . . . . . . . . . . . 55

5.2.1 Experiment Environment . . . . . . . . . . . . . . 56

5.2.2 Results of Resource Overhead Measurements . . . . . . . . 56

5.3 Chapter Summary . . . . . . . . . . . . . . . . . . 58

6 Identification of Critical Resources $\quad 61$

6.1 Introduction . . . . . . . . . . . . . . . . . . . 61

6.2 Computation of IF . . . . . . . . . . . . . . . . . . 62

6.2 .1 Impact Factor . . . . . . . . . . . . . . . . . . 62

6.2 .2 Weighted IF . . . . . . . . . . . . . . . . . . . 64 
6.3 Instruction Frequency . . . . . . . . . . . . . . . . . . 65

6.3.1 Testing Plan . . . . . . . . . . . . . . . . 65

6.3.2 Hardware Debugging Tool . . . . . . . . . . . . . 66

6.3.3 The Measured Results of Instruction Frequency . . . . . . 66

6.4 Access Frequencies of Register/Bit . . . . . . . . . . . . 69

6.4.1 IF Measurements . . . . . . . . . . . . . . . . . . . 70

6.4 .2 Test Flow . . . . . . . . . . . . . . . . . . 71

6.4 .3 Test Automation . . . . . . . . . . . . . . . 72

6.4.4 The Measured Results of Access of Register/Bit . . . . . . 74

6.5 The Results of Critical Resource Measurement . . . . . . . . . . . 75

6.6 Chapter Summary . . . . . . . . . . . . . . . . 77

$\begin{array}{lll}7 & \text { Discussions and Conclusion } & 79\end{array}$

7.1 Summary and Results . . . . . . . . . . . . . . . . . . . 79

7.2 Future Work . . . . . . . . . . . . . . . . . . . . . 80

$\begin{array}{lr}\text { References } & 81\end{array}$

A Critical Resource $\quad 85$ 



\section{List of Figures}

2.1 MOSFET Structure $[29] \ldots \ldots$. . . . . . . . . . . . . . 8

2.2 Change in N-MOSFET Gate Voltage from 500 krad Radiation [25] 8

2.3 Single Bit Upset and Multiple Bits Upset [6] . . . . . . . . . . . 9

2.4 Timing Requirements for SET to Occur [17] . . . . . . . . . 10

2.5 Impacts of Aluminum Shielding on TID [21] . . . . . . . . . . 12

2.6 Comparison of RHBD Cell with Standard CMOS Cell [3] . . . . . 13

2.7 Temporal Latch Functional Block Diagram [3] . . . . . . . . . . . 14

2.8 DICE Latch Diagram [3] . . . . . . . . . . . . . . . . . . . . . 14

2.9 TMR Functional Block Diagram [3] . . . . . . . . . . . . 15

2.10 Buffers adding to logic duplication $[36] \ldots \ldots \ldots$

2.11 Buffer gate with temporal redundancy [36] . . . . . . . . . . . 16

3.1 Structure of sensor design _ . . . . . . . . . . . . . . . . . . . 20

3.2 Normal process . . . . . . . . . . . . . . . . . . . . . . . . 20

3.3 Transient does not cause soft error . . . . . . . . . . . . . 20

3.4 A pulse that causes soft error . . . . . . . . . . . . . 21

3.5 False alarm on the XOR gate's output . . . . . . . . . . . 22

3.6 Design of soft error detection and recovery . . . . . . . . . . . 22

3.7 Design of sensor network . . . . . . . . . . . . . . . . . . 23

3.8 An overview of the LEON2 processor . . . . . . . . . . . . 25

3.9 The design of the error recovery scheme . . . . . . . . . . . 26

3.10 Error recovery waveforms . . . . . . . . . . . . . . 28

3.11 The LEON3 Structure . . . . . . . . . . . . . . . . . . . . . . . . 29

3.12 The edge detection algorithm . . . . . . . . . . . . . . 30 
4.1 Structure of the system . . . . . . . . . . . . . . . . 34

4.2 The normal execution result . . . . . . . . . . . . . . . . 37

4.3 Execution results with error injection but no recovery . . . . . . . 38

4.4 Execution result with error injection and recovery . . . . . . . . . 39

4.5 The software of automation test . . . . . . . . . . . . . . 40

4.6 The waveform of the result . . . . . . . . . . . . . . . . . 42

5.1 The test scheme . . . . . . . . . . . . . . . . . 47

5.2 The Measurement flow . . . . . . . . . . . . . . . . . . . . . . 48

5.3 Core0 process flow chart . . . . . . . . . . . . . . . 51

5.4 Core1 process flow chart . . . . . . . . . . . . . . 52

5.5 GRMON command snapshot . . . . . . . . . . . . . . 53

5.6 UART console snapshot . . . . . . . . . . . . . . . . . . 54

5.7 Time penalty distribution bar chart . . . . . . . . . . . . 55

5.8 The total resource usage . . . . . . . . . . . . . . . . . . 57

5.9 The maximum clock frequency and power usage . . . . . . . . . 58

6.1 Instruction Usage Diagram . . . . . . . . . . . . . . . . . 69

6.2 Automated Test Flow Chart . . . . . . . . . . . . . . . . . . 73

6.3 Automated Test Snapshot . . . . . . . . . . . . . . . . . . 74 


\section{List of Tables}

4.1 Random Error Injection Test . . . . . . . . . . . . . . . . . . . . 40

5.1 Time penalty of all test case . . . . . . . . . . . . . . 54

5.2 Resource Usage . . . . . . . . . . . . . . . . . . . . 58

6.1 Instruction timing $[13] \ldots \ldots$. . . . . . . . . . . . 64

6.2 Instruction Usage Statistic . . . . . . . . . . . . . . . . . 67

6.3 Register/Bit Number . . . . . . . . . . . . . . . . . . . 70

6.4 The Part of Access Condition of ACC Stage Register/Bit . . . . . 75

6.5 IF of All Registers . . . . . . . . . . . . . . . . 76

A.1 Critical Register Statistic of All Register . . . . . . . . . . . . 85

A.2 DE Stage IF Statistic . . . . . . . . . . . . . . . . . . . . . 92

A.3 ACC Stage IF Statistic . . . . . . . . . . . . . . . . . . . 92

A.4 EX Stage IF Statistic . . . . . . . . . . . . . . . . . 93

A.5 ME Stage IF Statistic . . . . . . . . . . . . . . . . . . . 94

A.6 X Stage IF Statistic . . . . . . . . . . . . . . . . . . . . 94

A.7 WB Stage IF Statistic . . . . . . . . . . . . . . . . . . 95 



\section{Chapter 1}

\section{Introduction}

\subsection{Computer System for Satellites}

Space exploration has been a dream of the human race for centuries. As the vanguards of humans, thousands of satellites have been sent into space since the first launch in 1957 [32]. Various kinds of satellites affect our daily life in many aspects, including weather forecast, televisions, telephones, etc. Satellites have been helping us in exploring space and giving us a better understanding of our planet. With the increasing demand for space exploration, satellites and various space applications are under intensive development. In all these applications, on-board computing systems play a key role for the entire application, because the satellites depend on the computer system to process the data, communicate with the ground station, and generating controlling signals for the satellite itself.

\subsection{Soft Errors}

The outer space is full of hazards such as high energy particles. For electronic devices, the high energy particles could cause electron-hole pairs which lead to malfunctions. For instance, the particles may flip the state of a logic device, such as a latch on memory cell [37]. Since the changed state does not cause the permanent damage to the logic devices, this unintended change of state is called a soft error. Soft errors could cause serious problems to the computer system. For 
instance, in the inertial navigation systems of satellites, the computer collects data from accelerometers and gyroscopes, and computes the location and the gesture of the satellites. If there is any soft error in the navigation system, it might cause incorrect gesture detection which could lead to the crash of the satellite.

\subsection{Previous Solutions}

For the computer industry, soft errors have been making great impacts. In 2000, the flagship Enterprise server line of the Sun Microsystems crashed due to the unprotected cache memories hit by cosmic rays [5]. To protect similar incidents from happening, Fujitsu protects $80 \%$ of 200,000 latches in its SPARC processor [1].

To improve the reliability of the computer system, various fault tolerant designs have been proposed.

The first kind of designs is the special radiation-hardened circuit design (e.g. [9]) which aims to raise the threshold voltage of a transistor, such that for the same amount of extra charges caused by high energy particles, it will not be easy to flip the state of the transistor. Another kind of designs is to localize error detection and correction (e.g. [1]). One popular design is architectural redundancy (e.g. [35], [23], [27], [2], [18]).

Generally, fault tolerant designs use either spatial or time modular redundancy [22]. With hardware redundancy, multiple hardware copies are used to process the same task. The final output is determined by a majority vote. With time redundancy, a task is executed for multiple times [17, 26, 28]. The extra time is used to verify the correct result. We can see that 2-3 times of area or performance overhead is sacrificed to achieve the enhancement of the system reliability.

To prevent soft errors from changing the states of latches and flip-flops, a robust system called "BISER" is proposed by Mitra et al. [16, 28]. Compared to the conventional Triple Modular Redundancy (TMR), the scheme reduces the cost significantly by reusing an on-chip scanning resource. However, the penalties on performance and energy are large. A multiple clocking of data technique [4] is 
proposed to dynamically detect and remove a soft error from the critical circuit path with a relatively low area of overhead. However, the pure hardware solution faces scalability problem, hence it is limited to protection of smaller systems.

Izoimov et al. [14] present an optimized fault tolerance method by selectively placing the redundant hardware and software re-execution. For a given application, they showed how to minimize the system cost while meeting the required level of fault tolerance. However, this method is still based on fixed protections against transient soft errors. Fixed protections are designed for worst cases which means they only account for a very small portion of the total system running time. This makes the fixed protection circuits a burden most of the time.

\subsection{Objectives and Approach}

This thesis is originated from a hardware-software collaborated fault tolerance project. In this project, we design, implement and verify a new soft error tolerant design for satellite applications. The aim is to enhance the reliability of a computer system with less area and performance overhead compared to existing approaches. In this thesis, we only focus on the hardware design, implementation and verification.

The objectives and goals are briefly summarized as follows:

- Implement the dynamic soft error detection and error recovery scheme on hardware

- Verify the correctness of the design

- Determine the most critical registers of a processor in order to optimize the fault tolerant design.

- Determine the performance, power consumption and resource usage of the new scheme

The new fault tolerant design includes two parts: sensors network and an error recovery scheme which is also called rollback scheme. The design of sensors 
network incurs only a small amount of extra circuits for detecting soft errors. The rollback scheme recovers the system from a soft error by re-executing the current instruction. The fault tolerant design will be prototyped and tested on LEON series processor. After the verification of the new fault tolerant design, we could determine the resource usage, power consumption and the performance of it.

To evaluate the overhead of the scheme, we determine the time and resource penalties of our scheme. The test results show that the scheme incurs only one extra clock penalty in about $90 \%$ of test cases and increases $0.282 \%$ of resource usage of the original processor for adding one 32-bit sensor. To identify the critical resource for protection, we define the weight of instructions based on the frequency of instruction usage. Moreover, we monitor the number of accesses of the internal registers/bits in LEON3. Then we compute the impact factor (IF) for each internal register and status bit according to the register access frequencies and instruction weight. Using this approach, we could figure out the most critical resources according to the impact factor. The results show that there are 233 registers and status bits in total. Of which, 91 of them have $100 \%$ IF which suggests they are the critical resources. 43 of them have an IF ranging between $1.9 \%$ and $97.6 \%$, which means they are less important. The IF of the remaining 89 is 0 because they are not used in our target application.

\subsection{Thesis Organization}

The rest of the thesis is organized as follows:

- Chapter 2 presents a literature review about the radiation environment of space and its effects on electronic devices, and the typical solutions for dealing with these effects.

- Chapter 3 presents the design of our soft error fault tolerant design and its verification method.

- Chapter 4 presents the verification methods on LEON2 and LEON3 processors. 
- Chapter 5 presents the evaluation of the overheads of our fault tolerant design, including the performance penalty, the resource overhead, and the peak frequency overhead.

- Chapter 6 presents the method for determining the critical resource of a processor according to the impact factor.

- Chapter 7 summarizes and discusses the work in this thesis and suggests the possible work in future. 



\section{Chapter 2}

\section{Literature Review}

In this chapter, we review the literatures about the impacts of cosmic radiation in space in Section 2.1 and review the recent solutions for dealing with radiation effects in Section 2.2.

\subsection{Radiation Effects on Electronic Devices}

\subsubsection{High Energy Particles}

Three types of charged particles in space are identified as they may cause the error to avionics: heavy ions, protons, and electrons. Energy level of electrons are an order of magnitude lower than the other types of charged particles, and electrons can be mitigated by minimal metal shielding, such as $1 \mathrm{~mm}$ of Aluminum shield. Thus, only protons and heavy ions are potential hazards. High energy particles could result in two types of radiation effects on electronic devices: permanent damage and soft errors. A permanent damage alters the voltage threshold of a transistor so that it could lead to a permanent device failure. The soft errors only temporarily impact the status of a transistor's gate, e.g., turning an "on" transistor "off", resulting in the logic level error. In this thesis, we will only focus on soft errors. 


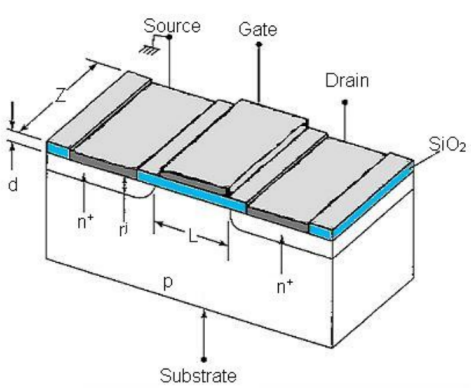

Figure 2.1: MOSFET Structure [29]

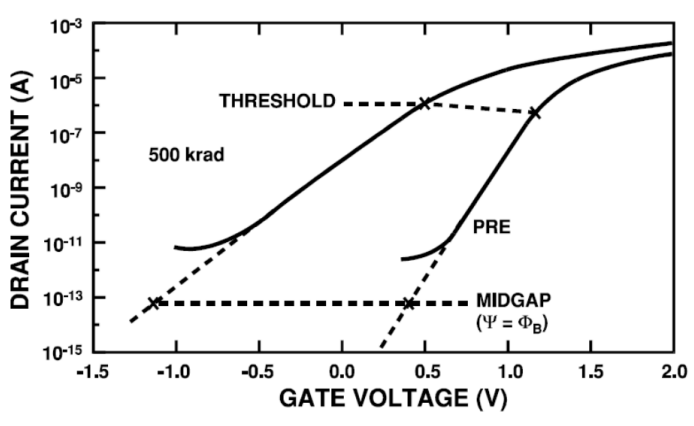

Figure 2.2: Change in N-MOSFET Gate Voltage from 500 krad Radiation [25]

\subsubsection{Total Ionizing Dosage Effects}

Fig. 2.1 is a MOSFET's structure, which is the basic element of an electronic device. The threshold of a gate, for a Si-based device, is about 0.7 volt. Shown as the line on the right in Fig. 2.2, with the gate voltage increasing, the drain current increases as well. In the radiation environment, the charged particles could accumulate in the $\mathrm{SiO}_{2}$ regions (the blue layer in Fig. 2.1) of a device. As charged particles accumulate, as the line on the left in Fig. 2.2 shows that a transistor's threshold is shifted to a lower voltage. When the dosage of the charged particles surpasses a certain level, it could alter the logical state of this gate. This effect is referred to as the total ionizing dosage effects.

\subsubsection{Single Event Effects}

Single Event Effects (SEE) include the effects of a semiconductor device interacting by high energy particles. The high energy particles create electron-hole pairs when passing through the substrate. A transistor gate must accumulate sufficient charges $\left(Q_{c r i t}\right)$ to reverse the current state. $Q_{c r i t}$ is determined by the operational voltage of the transistor and the transistor's gate size. Eq. 2.1 calculates the charge needed for a standard transistor while Eq. 2.2 is for circuits with active feedback against upsets [6]. Eq. 2.1 and Eq. 2.2 denote that as process sizes shrink (capacitance decreases) and the operational voltage decreases, $Q_{c r i t}$ also decreases.

$$
Q_{\text {crit }}=C_{\text {node }} V_{\text {node }}
$$




$$
Q_{\text {crit }}=C_{\text {node }} V_{\text {node }}+\tau_{\text {switch }} I_{\text {restore }}
$$

$$
\begin{gathered}
C_{\text {node }}=\text { Capacitance between transistor nodes } \\
V_{\text {node }}=\text { Operating voltage of transistor nodes } \\
\tau_{\text {switch }}=\text { Logic switching delay time } \\
I_{\text {restore }}=\text { Feedback current supplied }
\end{gathered}
$$

SEE can be categorized as follows [3]:

\section{- Single Event Upset (SEU)}

The most common error caused by SEE is single event upset. There are two types of SEU shown in Fig. 2.3.

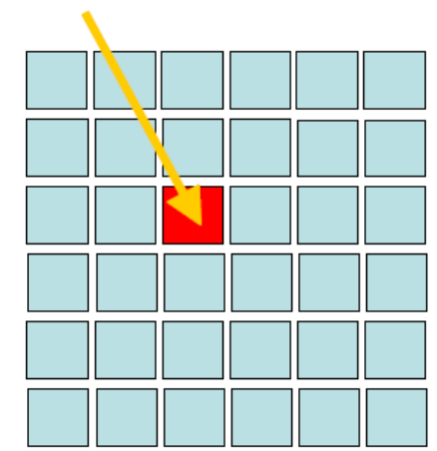

(a) Single Bit Upset

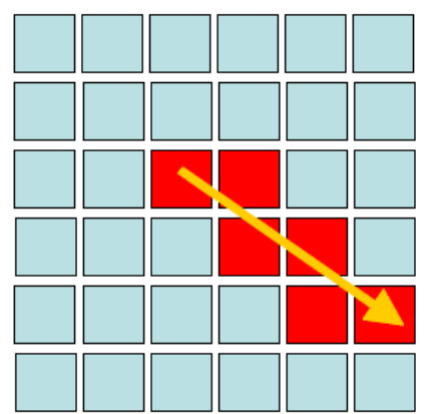

(b) Multiple Bits Upset

Figure 2.3: Single Bit Upset and Multiple Bits Upset [6]

As shown in Fig. 2.3(a), if a single energized particle changes the state of a single bit, it is called single bit upset (SBU). If the particle changes the state of multiple bits, as shown in Fig. 2.3(b), it is called multiple bits upset (MBU). SBU is commonly handled by utilizing error detection and correction (EDAC) codes. MBU can also use EDAC algorithm to cover errors, but it is usually ignored by designers due to the extremely low probability of MBU and the overhead of the EDAC algorithm on MBU.

\section{- Single Event Transient (SET)}

A single event transient is a temporary change in the logic level for a device. 
If this transient pulse is latched into a register, it becomes an SEU. Since most SETs have a small amplitude and a short duration, it usually appears as noises on a signal. Fig. 2.4 demonstrates the timing dependencies of an SET and a transient pulse.

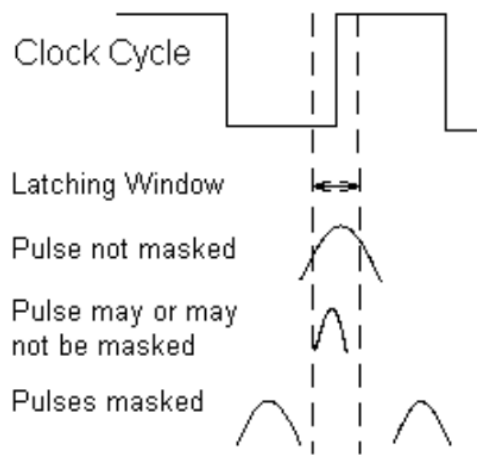

Figure 2.4: Timing Requirements for SET to Occur [17]

Every register has a latching window, in which it stores the combinational logic's value into a register. If a transient pulse spans the width of the latching window, an SEU occurs. If the pulse occurs in the latching window, but its width is smaller than that of the latching window, this pulse may or may not cause an SEU. If the pulse occurs outside the latching window, this pulse will not cause an SEU.

\section{- Single Event Functional Interrupt (SEFI)}

A single event functional interrupt refers to a device's functionality loss caused by an upset of an internal memory element or a circuit. For example, a processor could completely lose functionality if one of its interrupt control registers is modified to point to an unimplemented interrupt routine. An SEFI could be corrected by rewriting the modified register or by power cycling the device.

\section{- Single Event Latch-up (SEL) and Single Event Burnout (SEB)}

A single event latch-up is a lockup of a functional circuit within a device. If an SEL results in a peak current in the device's power supply, and the peak 
current exceeds the current limit of a transistor, it may destruct an element which is referred to as a single event burnout (SEB). SEB usually occurs in power transistors. An SEL can only be recovered by power cycling the device, however, an SEB is classified as uncorrectable device failure.

\subsection{Solutions for Overcoming Radiation Effects}

As presented in the preceding section, the radiation effects on electronic devices include the Total Ionizing Dosage (TID) and the Single Event Upset (SEU). In the following sections, we will present the widely used solutions for overcoming TID and SEU.

\subsubsection{Overcoming Total Ionizing Dosage Effects}

In order to make sure that electronic devices in space could operate properly over the entire mission, it must be designed to handle the Total Ionizing Dosage (TID). The TID tolerance level of a device is generally determined by the process of the electronic device and its shielding.

\section{- Radiation Hardened by Process}

One main effect of TID is that the accumulated charge alters the gate threshold voltage which could change the state of a transistor. To deal with TID, a layer is usually inserted in the substrate to increase the probability of the excess charge. This process has a benefit of increased speed by about $30 \%$ and decreases power consumption by about $25 \%$ [10]. Techniques such as this, by modifying the standard wafer fabrication process to minimize the TID effects, are referred to as Radiation Hardened By Process (RHBP). RHBP is further accomplished by making a thinner gate oxides layer which could be naturally achieved as reduced feature size because of the advanced process. For example, 250nm feature size transistors can tolerate up to 100 krad dosage of radiation before permanent failure while $90 \mathrm{~nm}$ feature size transistors can withstand greater than $400 \mathrm{krad}$ [3]. 


\section{- Shielding}

Shielding is to place Aluminum or Tantalum around sensitive electronics to block charged particles from reaching them. As shown in Fig. 2.5, the

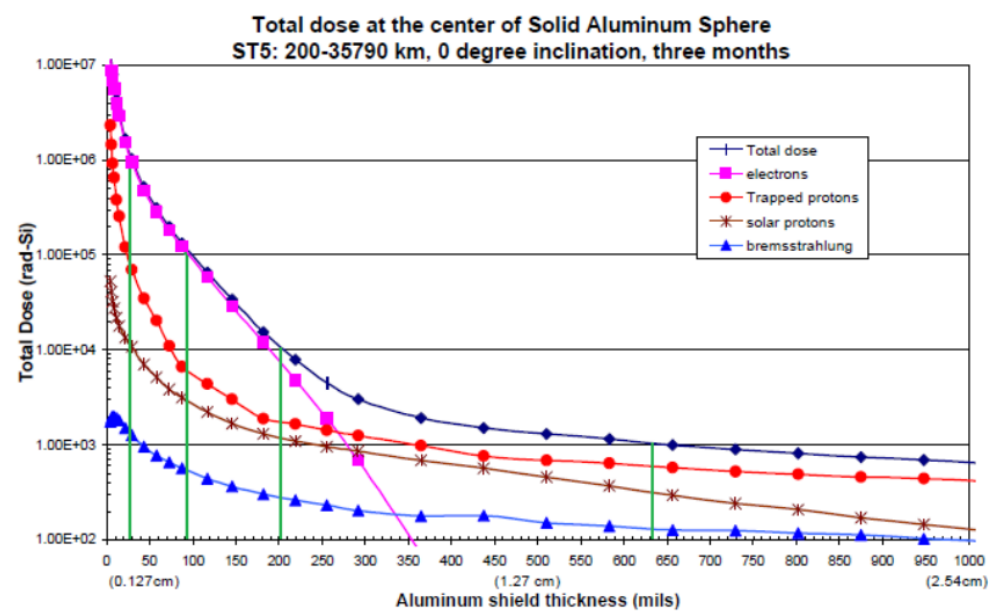

Figure 2.5: Impacts of Aluminum Shielding on TID [21]

absorbed dose is reduced by three orders of magnitude while using 200 mils shield. Additional shielding is less effective. As shown by the first green vertical line on the right in Fig. 2.5, the absorbed dose is reduced only by one orders of magnitude while the shield's thickness increases from 200 mils to 630 mils.

\subsubsection{Overcoming Single Event Effects}

\subsubsection{Radiation Hardened by Design}

Radiation hardened by design (RHBD) was developed to reduce the cost and development time of radiation hardened by process (RHBP), because the RHBP requires the modification of the standard wafer fabrication process which gains the extra cost and design time. RHBD uses standard silicon fabrication processes with specialized layout techniques to handle the SEE. Some common RHBD techniques are listed below: 


\section{- Increased Device Feature Size}

Since the feature size is linearly proportional to $Q_{\text {crit }}$ (as shown in Eq. 2.1), the most basic RHBD technique is to increase the feature size. Fig. 2.6 shows the effects of increased feature size on a two-input NAND gate. The RHBD cell (left) increases $25 \%$ of the area than a standard cell (center) and decreases $44 \%$ of the area than the standard cell from the previous generation (right). The increased feature size leads to a higher power consumption and a lower peak frequency [3].

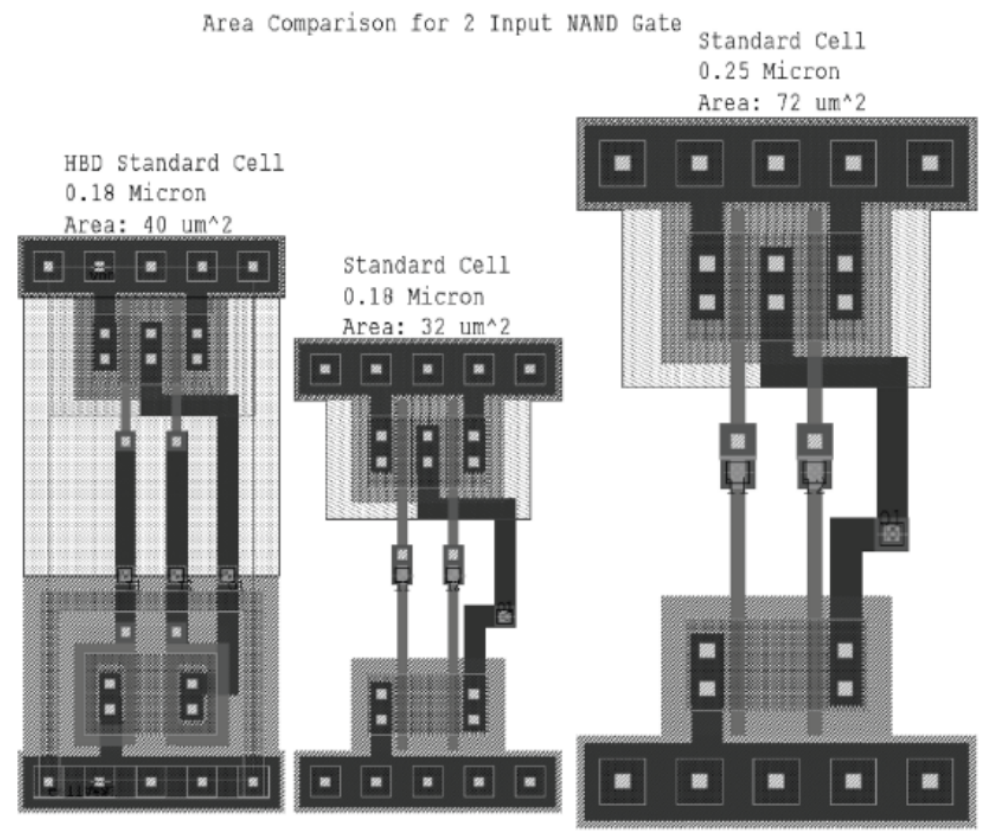

Figure 2.6: Comparison of RHBD Cell with Standard CMOS Cell [3]

\section{- Redundancy}

Another common RHBD technique is to add redundant circuits to increase the robustness of a system. The primary types of circuit level redundancy are discussed below:

\section{- Temporal Latch}

As shown in Fig. 2.7, two extra latches are used to store the combinational logic's value at delayed time of $\Delta T$ and $2 \Delta T$ and using the 
result of majority vote as the output [24]. However, since the delayed clocks extend the data path from the input to output, consequently, it decreases the performance and the peak frequency.

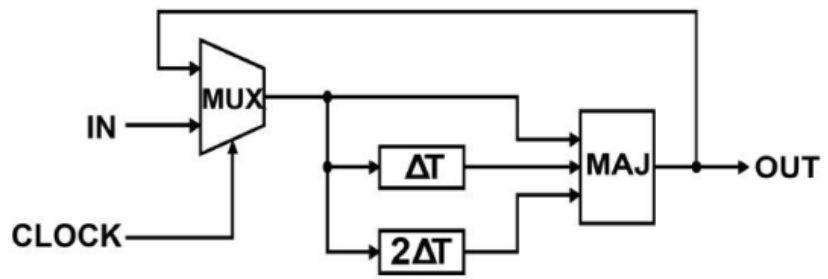

Figure 2.7: Temporal Latch Functional Block Diagram [3]

\section{- Dual Interlocked Cell (DICE)}

A dual interlocked cell is a replicated transistor circuit, storing a copy of the binary inverse of the data. Each transistor has a redundant partner to provide feedback control which ensures that a single upset will not change the circuit's output [20]. The transistor redundancy increases reliability, but it also increases area and power by at least $100 \%$.

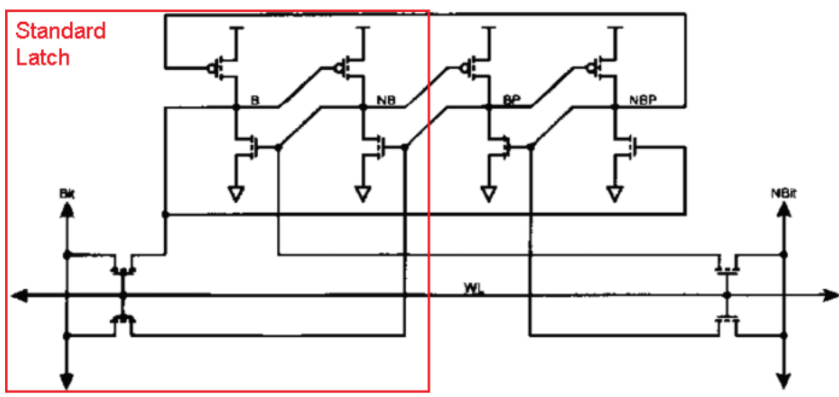

Figure 2.8: DICE Latch Diagram [3]

\section{- Triple Modular Redundancy (TMR)}

One classic method for implementing circuit level redundancy in space systems is TMR. As shown in Fig. 2.9, three identical modules send outputs to a voter for determining a majority [24, 34, 15]. If one 
output of module suffers from an upset, it is unlikely for the others to be affected because of the physical separation, and the majority of the voter is correct. Obviously, TMR increases power and area by at least three times.

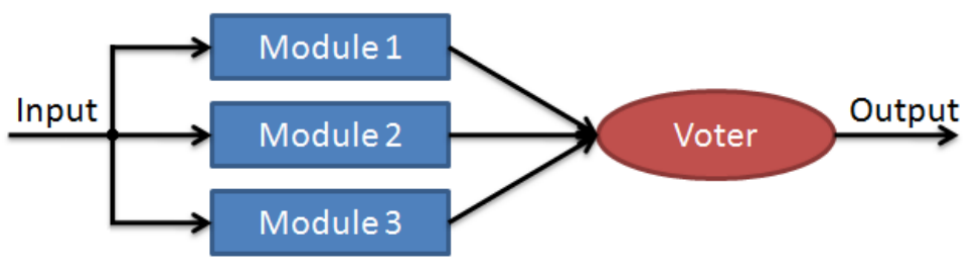

Figure 2.9: TMR Functional Block Diagram [3]

\section{- Logic duplication}

Logic duplication adds a buffer to prevent the SETs [36]. As shown in Fig. 2.10, two extra MOSFET are added as the buffer gate. The extra MOSFET usually consists two PMOS and two NMOS transistors. The output changes only when both inputs are the same. Therefore, it could prevent a wrong value from changing the output of the gate. However, even the overhead of logic duplication might be less than the TMR technique, the area is still large due to the duplication.

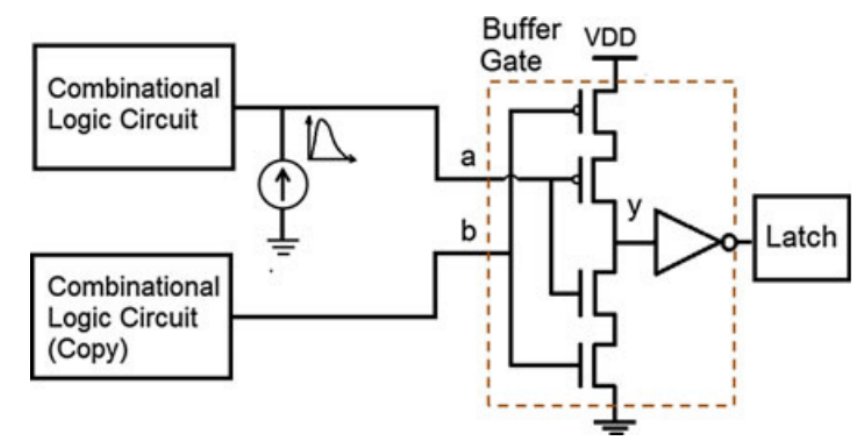

Figure 2.10: Buffers adding to logic duplication [36]

- Combination of temporal and spatial schemes

Fig. 2.11 shows a scheme that embodies both temporal redundancy 
and the logic duplication technique. In this design, there are two paths of data input. One path of inputs has no delay on the signal. Another path is delayed by a period of time. The output of the buffer is the same with logic duplication scheme, only changing the output when both inputs are identical. Moreover, the extra delay could avoid the transient of signal affecting the output. However, this scheme still has the disadvantage of the logic duplication, that is the large area.

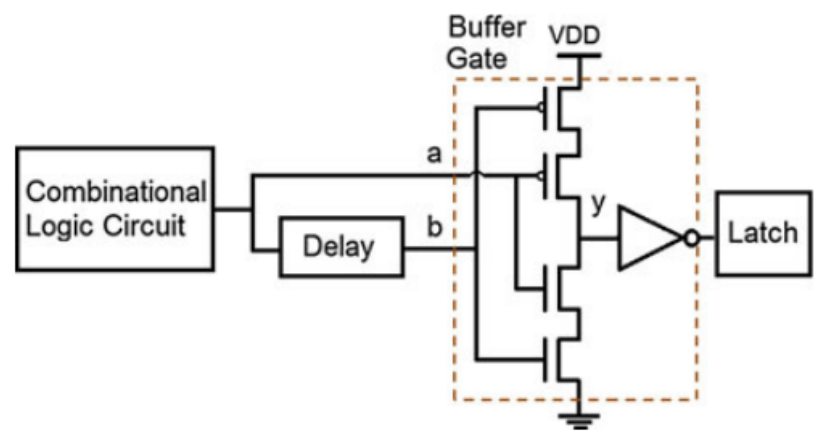

Figure 2.11: Buffer gate with temporal redundancy [36]

\subsubsection{Device Level Hardening}

Another approach is to apply TMR at the device level where each critical device is replicated. Boeing has created such a design using COTS PowerPC 750s for highperformance spacecraft payload operation [7]. Another design is called TimeTMR (TTMR) which is based on the TMR but improves the time performance. A TTMR system has the same design with TMR, however, it only compares the outputs of two modules. The third module is only used for mitigating errors. This allows two modules to continue operating while one module is reset because of an error. The modules operate in a round-robin fashion to increase the system performance [11].

\subsection{Summary}

In this chapter, we surveyed the contributors of radiation effects and categorized the existing methods for dealing with the radiation-induced errors including the 
manufacturing process and the design level. For dealing with the total ionizing dosage effect, one common approach is to harden the transistors during the manufacturing process or to add extra Aluminum or Tantalum shielding. To deal with the Single Event Effects (SETs), a typical way is to either increase device feature size or add redundant circuits. Increasing the device feature size requires the changing of the wafer process. This is very expensive and not flexible for designing because it needs different techniques for manufacturing.

In this thesis, we will only focus on circuit redundancy which can be divided into three categories. The first category is spatial redundancy techniques, such as TMR, TTMR and logic duplication. These techniques incur large area overhead because of the duplication circuits. The second category is temporal methods, such as the temporal latch. This method decreases the area usage but requires one or more delay elements and the voting unit which decrease the performance of the system. In addition, the extra delay elements and the voting unit also increase the area usage. The third category is the combination of the spatial and temporal method. This method balances the area and the performance by reducing the duplication and delay elements. Such design requires duplicating transistors at the circuit level, which is not easy to achieve for common fault tolerant designers.

We propose a new method which duplicates the registers instead of transistors to prevent the Single Event Transient (SET). Our design could reduce the area usage and increase the performance at the same time and avoid the difficulties that are associated with lower level circuit design. 



\section{Chapter 3}

\section{Design of Fault Tolerant Scheme}

In this chapter, we introduce the design of sensor network in Section 3.1 and the design of the error recovery scheme in Section 3.2. In the end, we make a brief summary in Section 3.3.

\subsection{Design of Sensors Network}

\subsubsection{Design of A Sensor}

A sensor is used to detect soft errors. Similar to the time redundancy circuit used in [22], the sensor design used in our project is shown in Fig. 3.1. The sensor includes two parts. The first part is a shadow flip-flop which is triggered by a delayed clock and used to sample the output of combinational logic. The second part is an XOR gate used to compare the output of the flip-flop and its shadow flip-flop. The two flip-flops capture the output of a combinational circuit of the same processing stage but at different points in time. If the values of two flip-flops are different, then XOR asserts.

Soft errors are caused by transient pulses on the output of the combinational logic or the data-path registers. If the transient pulse causes an unintended change to the value of a register, then a soft error occurs. Otherwise, the pulse only causes a glitch in the output of combinational logic. When the pulse passes, the output will go back to the correct value. For example, Fig. 3.2 shows a normal 


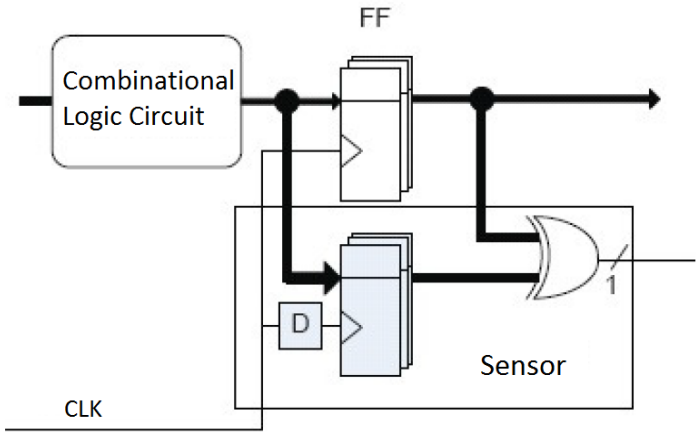

Figure 3.1: Structure of sensor design

processing of a flip-flop. At every rising edge of CLK, FF1 stores the value of the combinational logic's output.

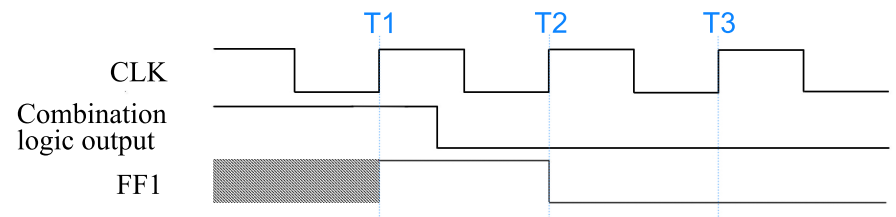

Figure 3.2: Normal process

Fig. 3.3 shows a transient at the combinational logic caused by high energy particles. This transient happens before the rising edge of CLK, so FF1 does not store the value of this transient. After the transient passes, the output of the combinational logic is stable again. In this case, FF1 operates normally, and no soft error occurs.

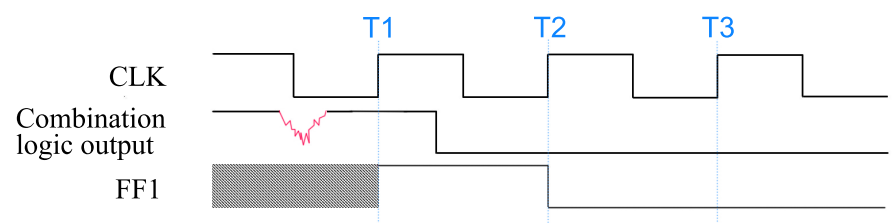

Figure 3.3: Transient does not cause soft error 
In Fig. 3.4, the transient pulse happens to be near the rising edge of the CLK, and FF1 stores the transient pulse "0" which is a wrong value. In this case, a soft error has occurred.

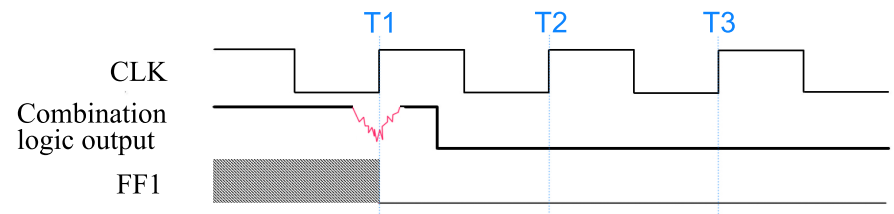

Figure 3.4: A pulse that causes soft error

In our sensor design, as long as the value of the shadow flip-flop differs from the monitored flip-flop, the XOR gate will be asserted, meaning, a soft error will be detected.

Since the shadow flip-flop is triggered by a delayed clock, as long as the clock delay is longer than the duration of the transient pulse, the soft error could be caught. At the same time, the clock delay cannot be longer than the shortest output path of the combinational logic, because the output will change to the next value and be captured by the shadow flip-flop if the clock delay is too long. Therefore, let $T_{s p}$ denote the shortest output path of the combinational logic, let $T_{l d}$ denote the maximum duration of the transient pulse and let $T_{d}$ denote the clock delay, the following inequalities must be satisfied:

$$
T_{s p}<T_{d}<T_{l d}
$$

The output of the XOR gate can generate "false alarm" glitches. In Fig. 3.5, D-CLK is a shifted clock of CLK, whereas FF1' is a shadow flip-flop of FF1, and FF1' will store the combinational logic's output at the rising edge of the D-CLK. FF1 and FF1' signals are compared by an XOR gate. The XOR gate could generate "false alarms", as shown in the circle of Fig. 3.5. It is because the shifted clock has a delay which causes FF1 and FF1' to have different values between time $\mathrm{T} 1$ and $\mathrm{T} 1$ '. In this period, the XOR gate will output " 1 " which is 


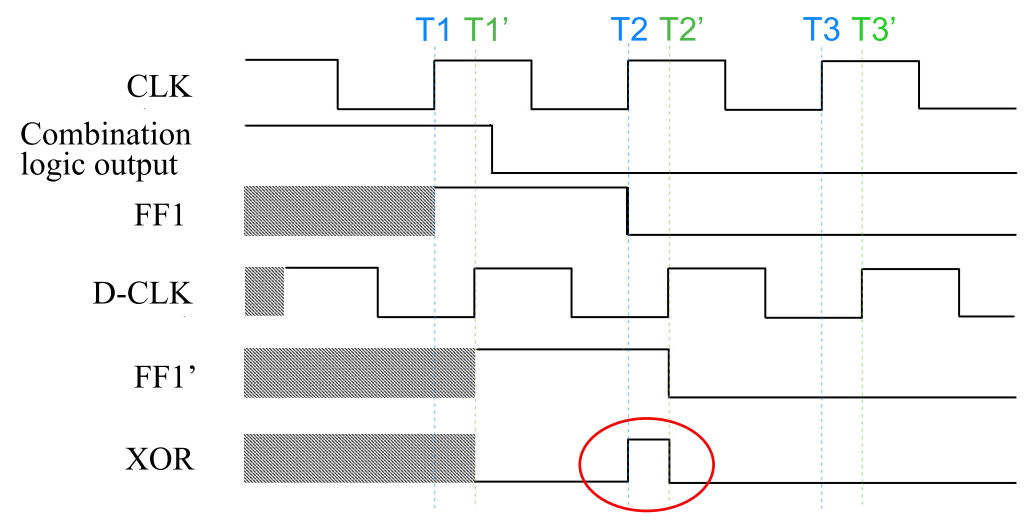

Figure 3.5: False alarm on the XOR gate's output

a "false alarm". To deal with this problem, we simply ignore the output of the XOR in between the rising edge of CLK and D-CLK.

The sensors in our design are only responsible for detecting soft errors. Some techniques use hardware to provide error-free circuit by integrating detection and recovery [16]. The design is shown in Fig. 3.6.

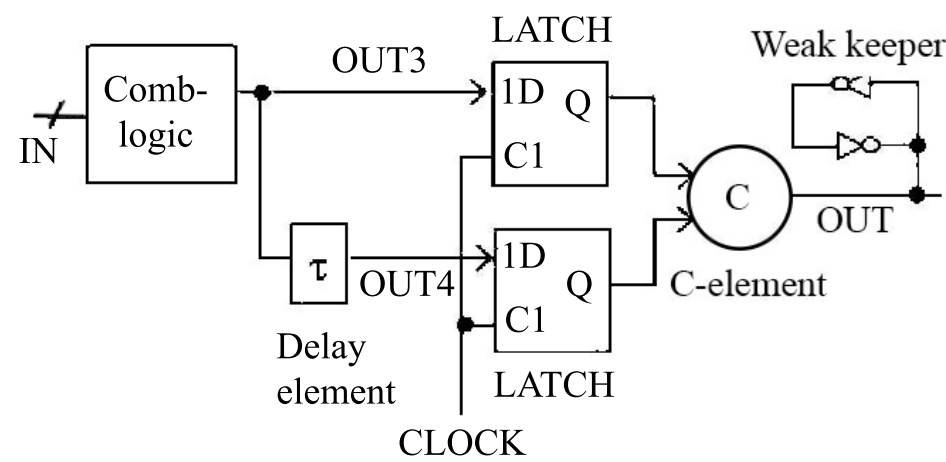

Figure 3.6: Design of soft error detection and recovery

It adds a C-element to perform the error recovery. The C-element outputs the inverted value if the value of the two flip-flops are the same; otherwise, according to the design of [16], it keeps the original value. The basic error-free flip-flop is built by this design of error detection and recovery. 
However, compared to our design, it has two disadvantages. First, the errorfree circuit has a longer delay, because it uses complex logic to implement a basic flip-flop. Second, because of the replacement, it changes the original design a lot and makes it hard to deal with the system integration. Whereas our sensor is a lightweight design, so it incurs very low hardware cost compared to the error-free design. Moreover, the lightweight design makes a minimum change on hardware designs, which makes it easy to integrate with the original system.

\subsubsection{Sensors Network}

Fig. 3.7 illustrates the design of the sensor network. In the design, a fixed number of sensors are distributed on a processor core. The number of sensors is determined by the number of registers in the data path of the processor core. Ideally, every register should be monitored by a sensor in the processor core. An OR gate is used to integrate outputs of all sensors.

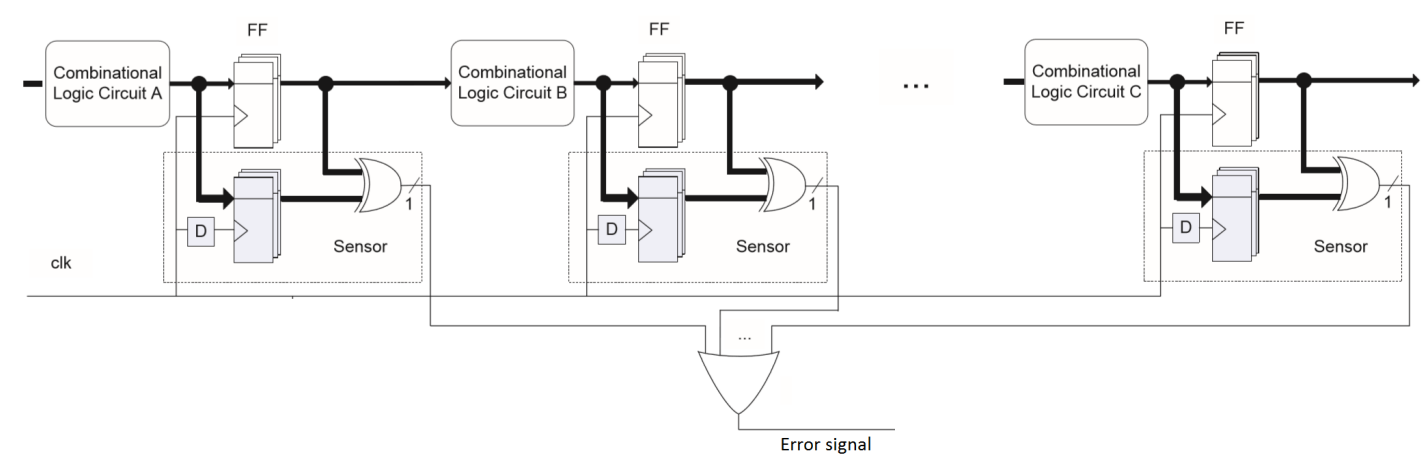

Figure 3.7: Design of sensor network

Any HIGH state from these sensors will make the OR gate enter a HIGH state as well. However, there may be a large number of sensors in a processor, which means lots of the sensors' output need to be integrated via a series of OR gates. The output of the OR gate in the final stage is called Error Signal, which could be utilized to inform the system that an error occurs. The utilization of the Error Signal will be described in Section 3.2.2. 


\subsection{Implementation of Error Recovery Scheme}

Field programmable gate array (FPGA) provides a rapid development and verification tool-chain for the implementation of our sensor and error recovery scheme. Moreover, since we need to modify the hardware of a processor to add the sensors and the fault tolerant designs, open source soft processor is the natural choice.

\subsubsection{Target Processor}

The LEON series soft processors were originally designed by European Space Agency (ESA) of European Space Research and Technology Centre (ESTEC) and used in the space missions for years [31]. The LEON series processors are 32-bit soft processors based on the SPARC V8 architecture. As an open source project, LEON processors provide synthesizable VHDL code and are highly customizable. They could be implemented on FPGA or manufactured into an Application Specific Integrated Circuit (ASIC). Therefore, LEON series processors are ideal for both research and commercial applications.

The LEON processor series can be categorized into the following families:

- LEON2 processor is the early version of SPARC core which has a 5-stage pipeline.

- LEON3 processor is a further improved core on the original SPARC V8 architecture, utilizing a deeper 7-stage pipeline and supporting multiprocessor design.

- LEON4 processor is an improved version of LEON3 with wider internal buses, modified pipeline and level-2 cache support.

Compared to other versions, the LEON2 processor is mature enough, but it is less complicated and easier to understand. Thus, we choose LEON2 to build the experimental platform to implement our soft error fault tolerant scheme first, then migrate the scheme to LEON3 processor. 


\subsubsection{Implementation on LEON2}

\subsubsection{Overview of LEON2 Processor}

Fig. 3.8 shows the structure of an LEON2 processor [12]. All components shown are configurable. We keep the initial design as simple as possible in order to reduce the complexity of the processor, and make it easy to implement and verify. Thus, we only utilize the 5 -stage integer unit with Cache, Debug support unit, memory controller, UART and I/O port in the LEON2 processor.

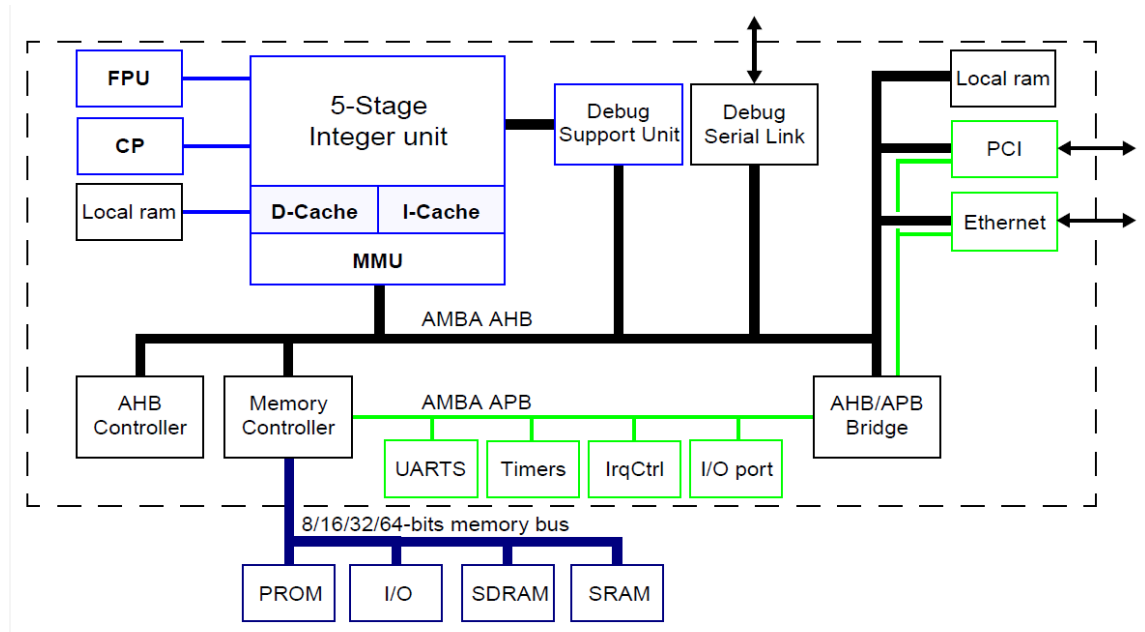

Figure 3.8: An overview of the LEON2 processor

Our main modification is in the 5-stage Integer Unit (IU). The IU is defined according to the SPARC architecture with five pipeline stages:

Instruction Fetch (FE): The Instructions Fetch stage fetches the instruction from the instruction cache (Icache) first or the memory if Icache misses.

Decode (DE): The Decode stage decodes the instruction from FE, calculates the next PC, and reads any operands required from the register file.

Execute (EX): The Execute stage executes the instruction, all Arithmetic and Logic Unit (ALU) operations are done in this stage. The ALU performs operations such as addition, subtraction, shift left and right, etc. 
Memory (ME): The Memory Access stage performs any memory access required by the current instruction. For loads, it would load an operand from memory. For stores, it would store an operand into memory. For all other instructions, it would do nothing.

Write (WR): The Write back stage writes the result of ALU back to the register file if the destination of this instruction is the register file.

\subsubsection{Implementation}

As described in Section 3.1.1, a sensor network is used to detect soft errors. The next step is to utilize the Error signal generated by the sensor network to fulfill the error recovery function.

After analyzing the source code of the LEON2 processor, we find that a series of special signals are used for synchronization between FPU, Icache, Dcache, and IU. As shown in Fig. 3.9, the solid lines and frames are the original synchronization design of the LEON2 processor.

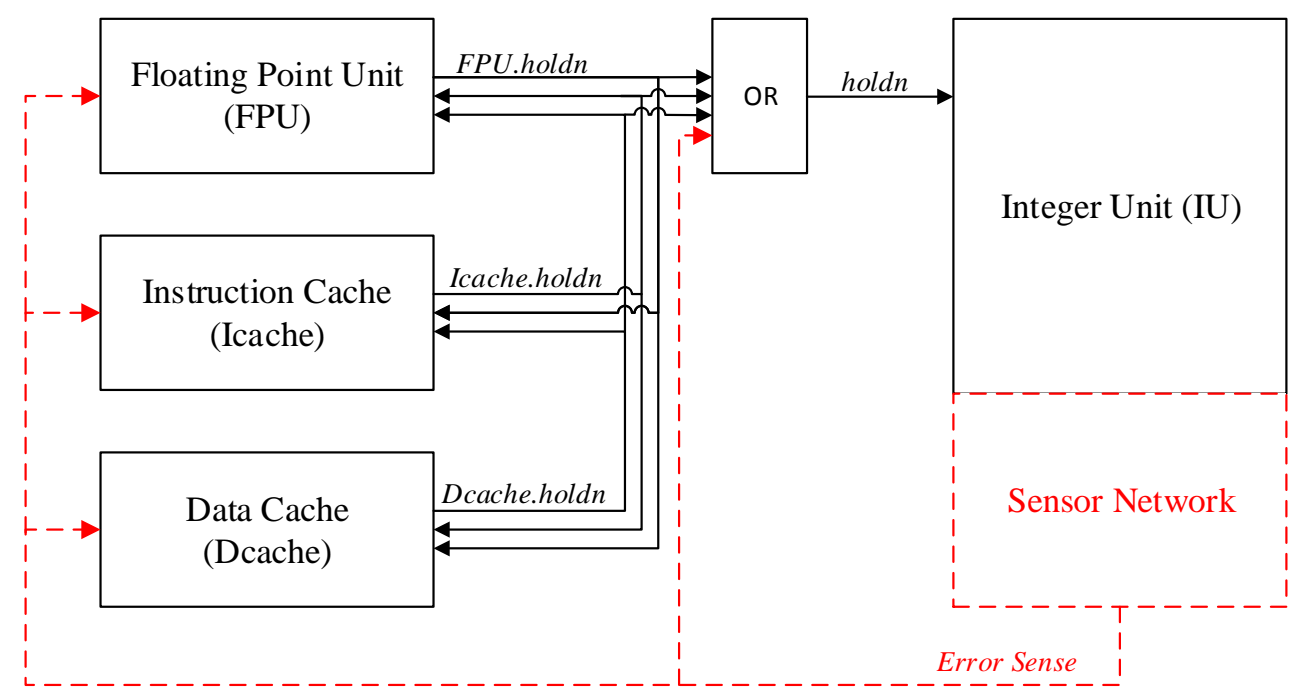

Figure 3.9: The design of the error recovery scheme 
FPU uses the FPU.holdn signal to inform the Icache, Dcache and IU that FPU is not ready, which might be because the calculation of floating point numbers needs more clock cycles.

For Icache, whenever there is a need (e.g., instruction cache miss) to load instructions from memory, Icache will assert the Icache.holdn signal to inform the other components. Similarly, Dcache performs the same synchronization scheme by asserting Dcache.holdn signal.

All these three kinds of special signals are integrated by an OR (effectively) gate to output a holdn signal which is used to stall the IU from further proceeding and wait for the holdn to be released.

By analyzing the details of how the holdn signal works, we find that the holdn signal does not gate the clock of IU. Instead, it stops the middle-registers of the pipeline from forwarding to the next stage. In other words, the IU repeats the same processes in the pipeline if holdn is asserted. Therefore, if we skip the current clock cycle when the soft error occurs and execute the same thing at the next clock cycle, the soft error cannot propagate to the next stage, and the error could be recovered in the next clock cycle.

By using the holdn signal, we add extra logic with the output of the sensor network to generate Error Sense, as the dashed lines and frames shown in Fig. 3.9, to imitate the function of the holdn signal. If a soft error is detected, the sensor network will assert Error Sense to let the FPU, Icache, and Dcache wait, and the soft error could be recovered by the next clock cycle. Error Sense will not cause synchronization problems, because the design utilizes the original synchronization mechanism of the processor.

At every clock cycle, before transferring the values of the pipeline registers, the error recovery scheme will check Error Sense first, if it is not asserted, then the processor works as usual; otherwise, if a soft error is detected, which will triggers the holdn signal, the processor will stop the data from moving forward in the pipeline and repeat the same process in the next clock cycle. Thus, the soft error could be recovered.

For example, as shown in Fig. 3.10, at time T1, suppose FF1 stores the wrong value "0" of the combinational logic's output and causes a soft error. At T1', 
FF1' stores the combinational logic's output again. Then FF1 and FF1' would have different values because of the soft error. Then Error Sense will be asserted at the same time T1'.

When Error Sense is asserted, the pipeline registers are stopped from forwarding, which means, the input of the combinational logic of the next stage will remain the same, and its output also remain the same as before. Thus, at T2, FF1 stores the value of combinational logic again, which is the correct value. At $\mathrm{T} 2$ ', FF1' stores the combinational logic's output again. At this time, FF1 and FF1' has the same value, so Error Sense goes low, and the processor proceeds normally.

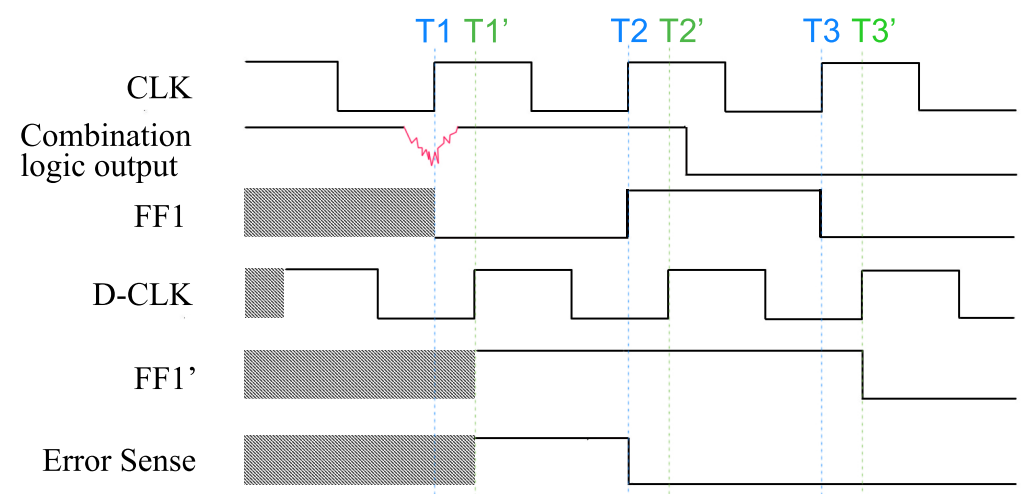

Figure 3.10: Error recovery waveforms

In this way, the soft error can be detected within the same clock cycle and be recovered in the next clock cycle. In order to make sure our scheme works in a real processor and will not cause any other side effects, we need to verify our scheme on LEON2. In the next section, we will describe the method of verifying the scheme and the result of the verification.

\subsubsection{Migration on LEON3}

\subsubsection{Overview of LEON3}

The LEON3 processor is also a SPARC V8 architecture based processor. Compared to LEON2, LEON3 has a 7-stage pipeline, which has an extra register-file- 
access (ACC) stage in between the DE and EX stages, and an extra exception (X) stage in between the ME and WB stages.

LEON2 and LEON3 are both based on the SPARC V8 architecture, hence their pipeline functions are similar. Indeed, after analyzing the source code of these two processors, we find that the function of the ACC stage in LEON3 is integrated with the EX stage in LEON2; and the function of the exception stage of LEON3 is integrated with the ME stage in LEON2.

Therefore, theoretically, our error detection and recovery scheme could easily migrate to the LEON3 because they both have similar architecture.

\subsubsection{Migration}

As shown in Fig. 3.11, it is the inner structure that we mainly used in an LEON3 processor. The sensor network is implemented in the 7-stage integer pipeline, and the error recovery scheme is implemented in between the IU, FPU, Icache, and Dcache. The trace buffer is responsible for storing the instructions history for further verification. The debug port provides the interface with the GRMON debugger to download applications and check the execution result. The interrupt port and AHB interface are for peripherals access.

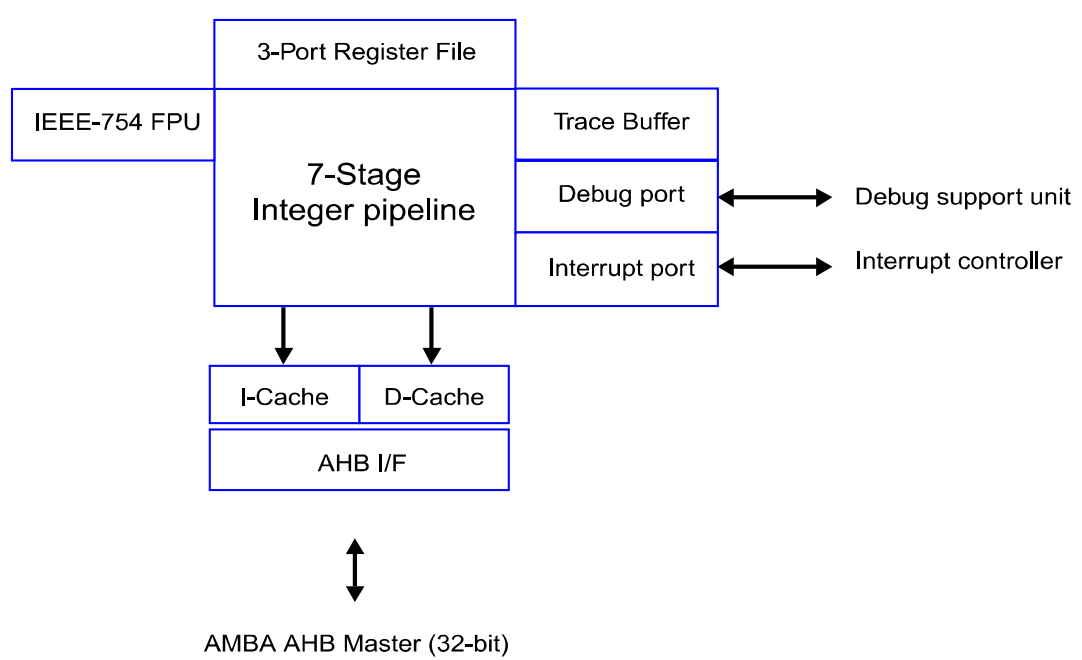

Figure 3.11: The LEON3 Structure 
By analyzing the RTL source code of the LEON3, the holdn scheme has some slight differences compared to LEON2, but the concept of synchronization between components remains the same. So after the implementation of the scheme, we run a normal application to test whether the processor works correctly after adding the sensor network and error recovery logic.

We run a Sobel edge detection algorithm on the LEON3 processor. Firstly, we download the application and a BMP figure onto memory. After the execution, we dump the content of the memory to a PC and convert it to a BMP image to check the result of the algorithm. Fig. 3.12(a) shows the original figure, and Fig. 3.12(b) shows the output of the algorithm. The Sobel edge detection algorithm is correct, which suggests that our implementation does not affect the original functions of the LEON3 processor. Therefore, the next step is to verify the correctness of our design on LEON3.

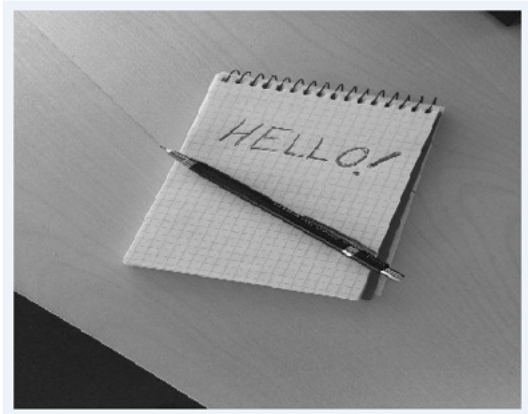

(a) Original figure

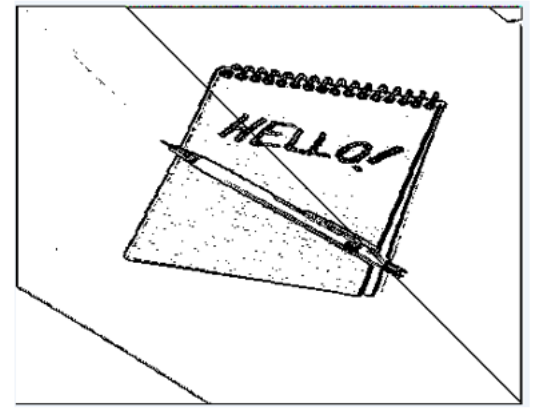

(b) Edge detection result

Figure 3.12: The edge detection algorithm

\subsubsection{Adding Error Information Register}

In order to gather detailed information about the experiments, we add two hardware counters to count the number of errors that occur and the corresponding performance penalty. We create a new register in LEON3 to store the the counters' value. We also add a new instruction to read this error register in a software application in runtime. 
The error register is added as a special register in LEON3, similar to the wim register, which keeps track of the current register window of LEON3. Following the connection and the structure of the wim register, we add a 32-bit register, called err register, to store the counter's value. The higher 16-bit of it stores the number of errors occurred, and the lower 16-bit stores the number of clock cycles incurred in the error recovery scheme (i.e., performance penalty).

We refer to the wim reading instruction, RDWIM, and create a new instruction called RDERR instruction to read the err register. By analyzing the compiler of the SPARC family's processor, we find that some OP codes are unused. Thus, we add a new OP code right after that of the RDWIM instruction, and the functional code analogous to the RDWIM instruction. In this way, the new instruction RDERR can be recognized by both the compiler and the processor. The utilization of RDERR instruction will be demonstrated in the following chapter.

\subsection{Chapter Summary}

In this chapter, we presented the design of one single sensor and the sensor network. Then we implement our sensor network both on the LEON2 and LEON3 processors. By utilizing the synchronization feature of the LEON processors, we implement the error recovery scheme on LEON processors. For further usage of information of error detection, we add error register to store the error counter and the penalty counter. We apply an image processing algorithm to ensure our sensors network and error recovery scheme have no impact on the functionality of LEON3 processor. 



\section{Chapter 4}

\section{Verification}

In this chapter, we verify the correctness of our sensors network and error recovery scheme. Section 4.1 illustrates the basic structure of the LEON processors. Section 4.2 and Section 4.3 describe the verification method and result based on LEON2 and LEON3 processors respectively. In the end, we briefly summarize the result in Section 4.4.

\subsection{System Design}

Fig. 4.1 illustrates the structure of the FPGA-based LEON processor.

The LEON processor is implemented on an FPGA, and the processor uses the Memory Interface Generator (MIG) IP to control the external memory. The configuration of the FPGA, including the implementation of the LEON processor, is stored in the configuration ROM. FPGA will configure itself upon powering up. PLL is used to provide the global clock for all the other components. Moreover, the LEON processor also could control the other peripherals, such as General Purpose Input/Output (GPIO), buttons, switches, LEDs, UART, etc. The LEDs represent the state of the system, and they also could be used for debugging. The UART transfers data between the processor and a PC. The buttons are used to interact with the processor, such as the error injection which we will discuss later. The switches could configure the different modes of the processor, such as the debug mode or the ordinary mode. 


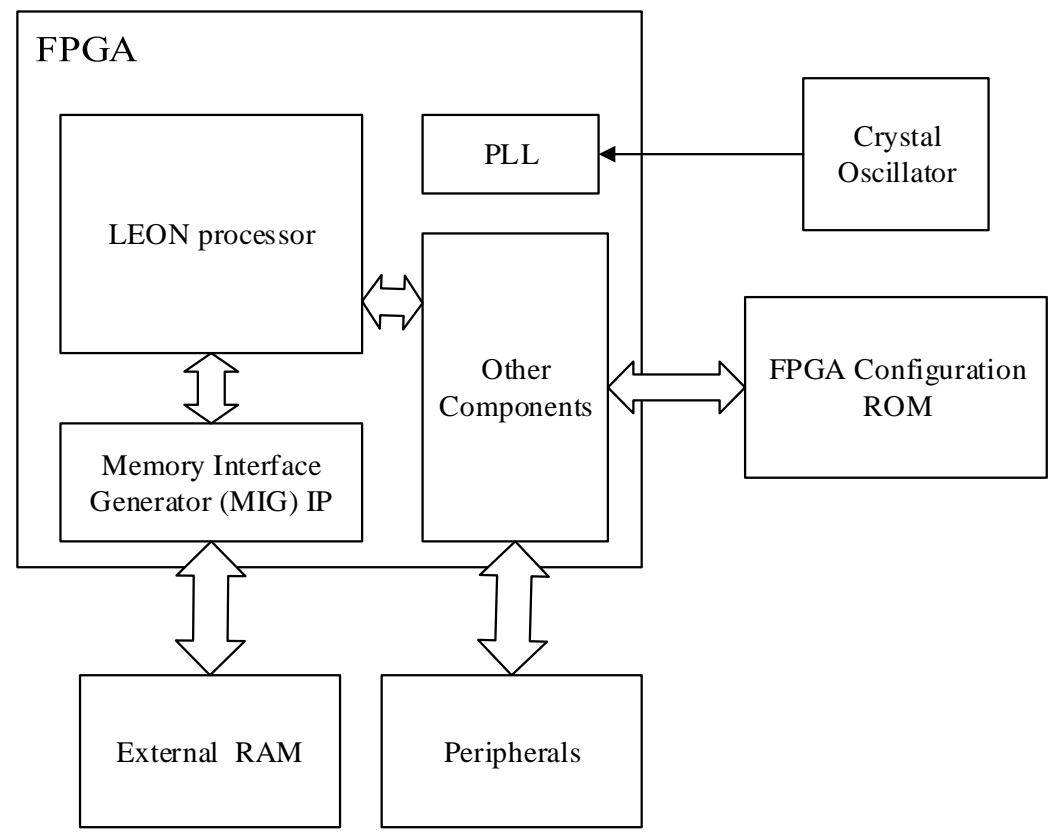

Figure 4.1: Structure of the system

\subsection{Verification on LEON2}

For verifying the correctness of the sensor network and the error recovery scheme, we implement the design to the Altera DE2 FPGA development board. The reason for choosing this older board is because the LEON2 processor is an older generation design which requires SRAM as the external memory. The DE2 board has a 2MB SRAM and meets all the other requirements of the LEON2's configuration.

\subsubsection{Tool Chain}

\subsubsection{GRMON Debug Tool}

GRMON is a general debug monitor for the LEON processor based on the GRLIB IP library and includes the following main functions:

- Downloading and execution of LEON applications 
- Read/write access to all system registers and memory

- Remote connection to GNU debugger (GDB)

- Built-in disassembler and trace buffer management

- Breakpoint and Watchpoint management

GRMON is used to download test applications, trace the execution history, and check the execution result.

\subsubsection{SignalTap II}

SignalTap II is an embedded on-chip logic analyzer for Altera FPGA chip family. It could integrate with an RTL design and capture the internal signals of a chip for debugging. In our test, the SignalTap II is used for capturing the waveform to check the status of an error injection and analyzing the data transitions of the pipeline registers.

\subsubsection{Verification Method}

For testing, we add sensors to the program counter (PC) of each pipeline stage, because the $\mathrm{PC}$ is responsible for fetching correct instructions, it is the most important registers.

For verification of the sensor and error recovery scheme, we need to inject errors in specific points of time and check the values of registers. The errors are injected to the shadow registers of PC because they are easier to access externally; moreover, the sensor can detect the soft error either on PC or on shadow PC.

Since every PC in the pipeline represents an instruction, we select a particular instruction to inject errors, and verify the waveforms before and after the error injection. The complete test flow is shown as follows:

- choose a target instruction by selecting the address with switches

- capture waveforms when the processor is executing the target instruction with one error injection 
- re-execute the application and inject error while the processor is executing target instruction on FE stage, and capture the waveform

- repeat the last step to capture the waveforms while the processor is executing target instruction on DE, EX, ME, WB stage respectively

- compare the waveforms and check if there is any wrong value

In order to cover a larger amount samples in our test, we firstly choose to verify the commonly-used instructions of the SPARC V8 Instruction Set Architecture (ISA), and then we inject error randomly to an application.

For a SPARC V8 processor, instructions can be listed into six basic categories [12]:

1. Load/store

2. Arithmetic/logical/shift

3. Control transfers

4. Read/write control register

5. Floating-point operations

6. Co-processor operations

Since the LEON2 implementation does not include Floating Point Unit and Co-processor, instructions of the categories 5 and 6 would not be considered.

For most of the instructions, their operators have two kinds of format, immediate and register, both of them need to be verified. Moreover, according to the verification steps, each instruction has six times of waveform capture. Thus, automation of the tests is the only choice.

For example, Fig. 4.2(a) shows the test of adding sensors to the PC of ME stage.

Fig. 4.2 shows the results of the normal execution. Fig. 4.2(a) is the screen shot of the GRMON which shows the execution history of the processor. The frame in Fig. 4.2(a) shows the target instruction st, which is used to store a byte of data to the memory. From the figure, we can also see that this instruction is finished at the 1502nd clock cycle and its address is 0x600011E4. 


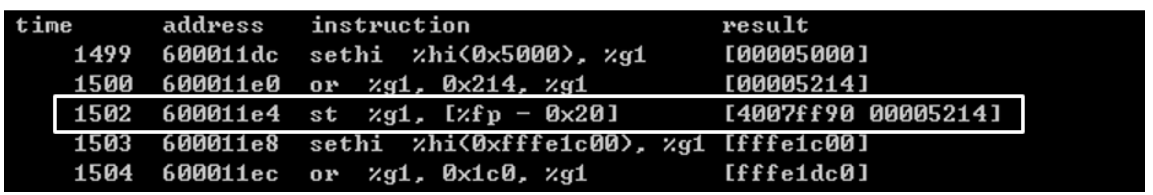

(a) Execution history of processor

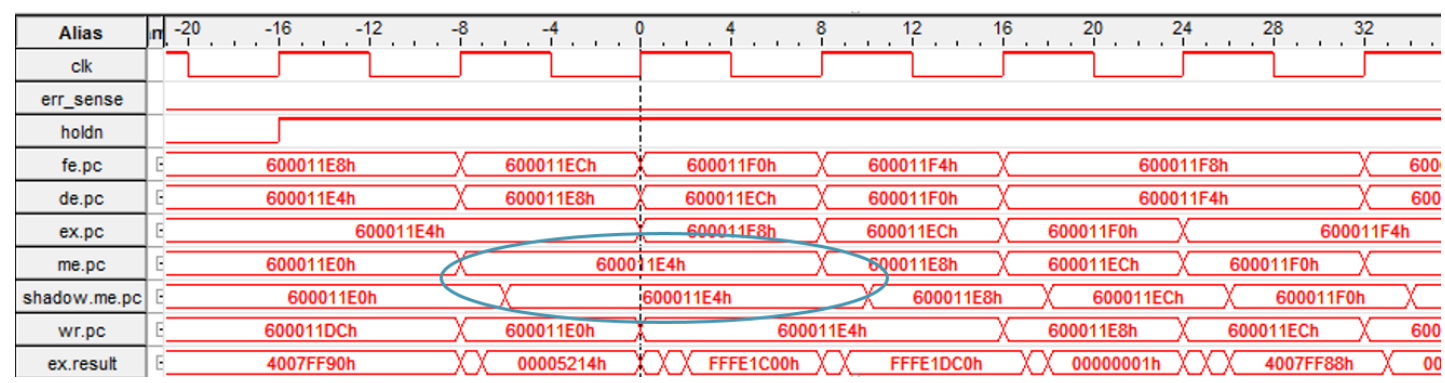

(b) The waveform screen shot

Figure 4.2: The normal execution result

Fig. 4.2(b) shows the waveforms of executing the target instruction. The circle in the figure shows that the PC of ME stage is 0x600011E4, and the shadow register changes its value a quarter clock cycle later than the $\mathrm{PC}$, because in our test, we use a 90-degree shifted clock as the delayed clock.

Fig. 4.3 shows the test result with error injection but no recovery. Fig. 4.3(b) shows the corresponding waveform. In Fig. 4.3(b), the No.2 circle indicates the error injected to the shadow PC, the value of which is changed from 0x600011E4 to 0x9FFEE1B. The error is detected by the sensor, and as indicated in the No.1 circle, the signal Error Sense asserts.

Fig. 4.4 shows the test result with error injection and recovery. As shown in Fig. 4.4(b), the No.3 circle highlights the error injected to the shadow register of the PC on the ME stage. Consequently, the error is detected by the sensor network and Error Sense asserts (shown in the No.1 circle). In this case, we enable the error recovery function, and Error Sense will control the holdn signal to stall the pipeline and re-execute the current instruction. As shown in the No.2 circle of Fig. 4.4(b), the holdn signal is pulled down by Error Sense, and the result is shown in the No.4 frame, the pipeline is stalled for one extra clock to execute the same instruction. From the preceding figures we can see, the st instruction normally costs two clock cycles and finishes at the 1502nd clock cycle. 


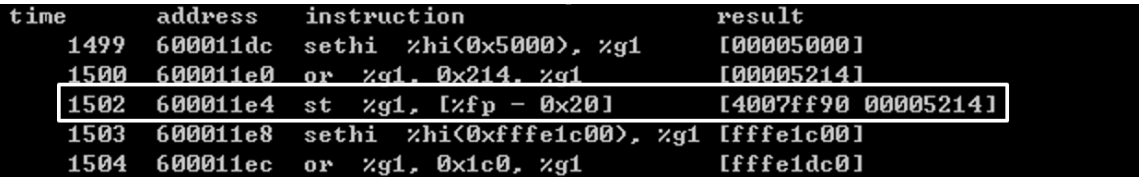

(a) Execution history of processor

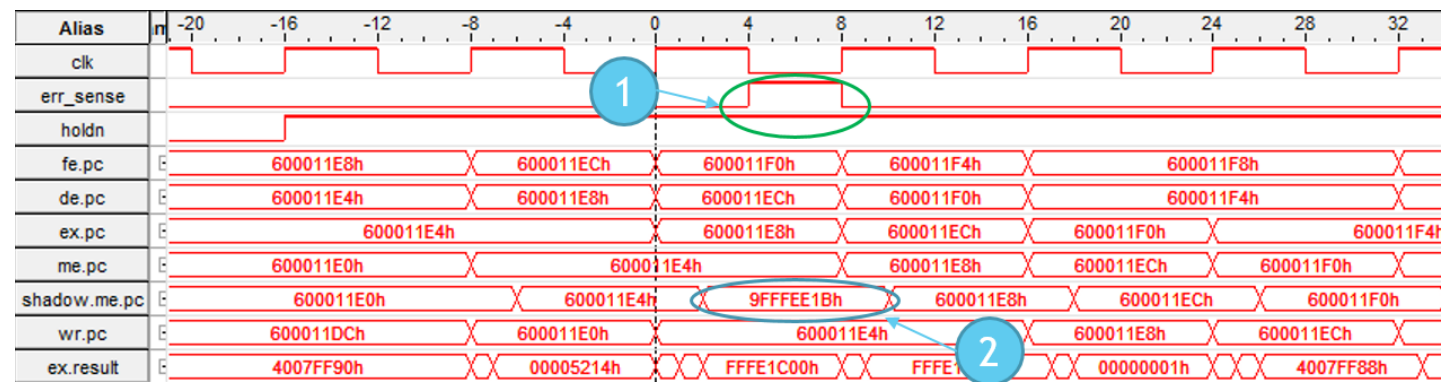

(b) The waveform screen shot

Figure 4.3: Execution results with error injection but no recovery

With the error recovery, however, Fig. 4.4(b) shows that the st instruction costs totally three clock cycles.

\subsubsection{Test Automation}

In order to reduce the workload of the verification, we designed a tool, as shown in Fig. 4.5 to automate of the testing work.

This tool uses GRMON, which is the debug tool of the LEON processor, to download the testing application of the target instruction, and to set breakpoint on a chosen address and send the execution control signals to the LEON2 processor. It then monitors the waveform of the SignalTap II. A soft error will trigger SignalTap II to capture the waveform. Then the tool will make a screen shot of the current waveform and the corresponding result in GRMON.

After the test of one instruction is over, the tool gives the next instruction's address and waits for the confirmation from the user. According to the information provided by the tool, we can change the value of the switches to the given address and make the tool repeat the test process again. 


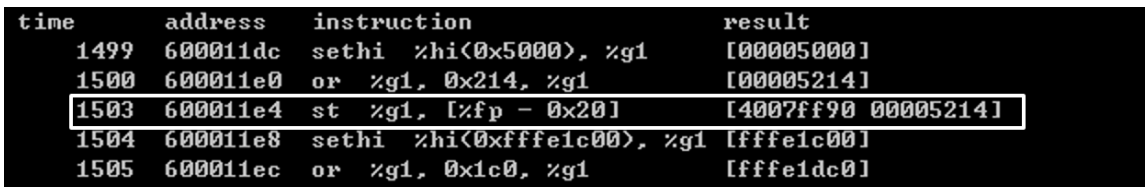

(a) Execution history of processor

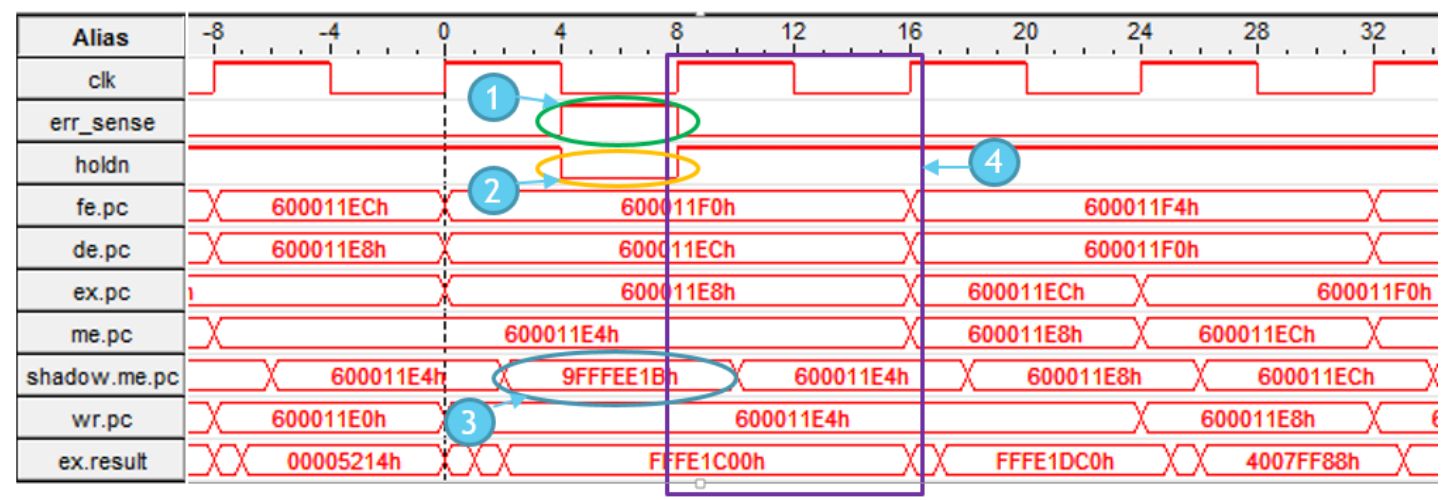

(b) The waveform screen shot

Figure 4.4: Execution result with error injection and recovery

\subsubsection{Verification Results}

In this section, we carry out two sets of tests. The first set is the SPARC V8 instructions set tests, and the second set is the random error injection tests on an application.

For instruction test, there are 166 different kinds of LEON instructions. Some of them are not used because of the configuration of the processor. 43 instructions are about floating point calculation; 24 instructions are about co-processing, and 39 instructions are for debugging usage. Excluding these instructions, only 60 instructions remain. Since some of them have two to four different formats, so the total number of instructions to be tested is 122. After analyzing the results, we find that using the sensor and rollback scheme, and all tested instructions have correctly executed in the presence of soft errors.

For random error tests, we use EEMBC's COREMARK benchmark as the application. A button is used to inject error during the execution of COREMARK. For each stage of the pipeline, we carry out three rounds of tests. As shown in Table 4.1, totally 766 errors are injected to the five stages of the pipeline, and all the results and the waveform are correct. 
Chapter 4. Verification

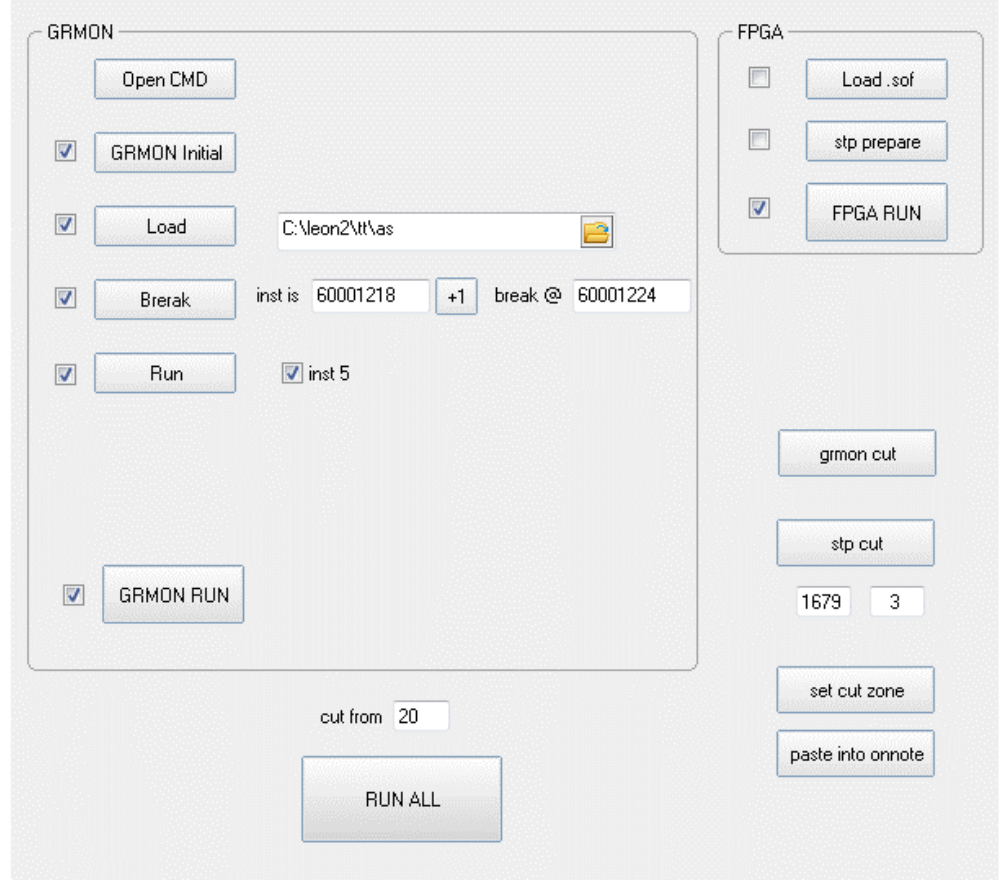

Figure 4.5: The software of automation test

In the instruction set tests and random error injection tests, all results are correct. The comprehensive tests suggest that our sensor network and error recovery scheme work correctly on LEON2.

Table 4.1: Random Error Injection Test

\begin{tabular}{ccccc}
\hline Stage & 1st round & 2nd round & 3rd round & Overall \\
\hline $\mathrm{FE}$ & 68 & 35 & 52 & 152 \\
$\mathrm{DE}$ & 53 & 54 & 52 & 159 \\
$\mathrm{EX}$ & 53 & 51 & 53 & 157 \\
$\mathrm{ME}$ & 64 & 36 & 46 & 146 \\
$\mathrm{WB}$ & 47 & 52 & 53 & 152 \\
\hline
\end{tabular}




\subsection{Verification on LEON3}

The verification on LEON3 is similar to what we did on LEON2. One of the slight difference is that we use ChipScope to capture the waveforms instead of SignalTap II. Because LEON3 is implemented on the FPGA of Xilinx company, and for Xilinx FPGAs, ChipScope provides similar functions. One major difference is that LEON3 has a 7-stage pipeline, whereas LEON2 only has five stages. This means we need to carry out two more tests for the extra pipeline stages.

LEON2 and LEON3 share the same instruction set because they are both based on SPARC V8 architecture. The instruction set tests have been done on LEON2, thus, we only carry out the random error injection test on LEON3.

To implement the LEON3 processor on an FPGA, we choose an Xilinx ML507 development board. The ML507 board has a 1GB DDR RAM, which could provide enough memory space to run larger applications or even an operating system. Moreover, it has enough hardware resource that not only allow us to implement our current design, but also to extend the design to multi-core fault tolerant system in future.

\subsubsection{Verification Method}

For testing, we add sensors to the program counter (PC) of each pipeline stage and inject errors in the shadow register of the exception (X) stage by pressing a button. Then we use ChipScope to check the waveforms, and use GRMON's result to verify the correctness of the results. The complete test flow is shown as follows:

- Execute the COREMARK application

- Randomly inject errors

- Capture the waveform by ChipScope and make a screen shot

- Analyze the waveforms and the result of GRMON to check if there is any wrong value 


\subsubsection{Verification Results}

Fig. 4.6 shows an example of the test. The left side shows the signal list. The first line is the main clock of the LEON3, clkm. The second line shows Error Sense which is high active. The third line is the holdn signal in LEON3, which is low active. The fourth line is the PC register of the FE stage, and the fifth line is its shadow register. The following signals are the $\mathrm{PC}$ and its shadow register of the DE, ACC, EX, ME and X stages respectively.

\begin{tabular}{|c|c|c|c|c|c|}
\hline Bus/Signal & $\mathrm{x}$ & 0 & $\frac{0}{T}$ & 5 & 15 \\
\hline /cllam & 1 & $1 \|$ & & & Ц \\
\hline -/pulse_gen/out0 & 0 & & & & \\
\hline - $/ 13$. cpu[0]. u0/1eon $3 \times 0 / p 0 /$ rollbackout_error_sens & 0 & & & & \\
\hline /13. cpu[0]. u0/1eor $3 \times 0 / p 0 / h o l d n x$ & 1 & & & S & \\
\hline o- /13. cpu[0]. u0/1eon3x0/p0/iu/r.f.pc & 40004 & 40004 政 & $0004 \mathrm{~B} 30 \times 40005 \mathrm{E} 6 \mathrm{C} \times 4 \mathrm{~d}$ & $40005 \mathrm{E} 74$ & $40005 \mathrm{E} 78$ 40005E $7 \mathrm{C}$ 40005F0C $40005 \mathrm{~F} 10$ \\
\hline 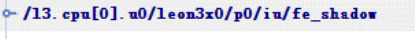 & 40004 & 40004 & $0004 \mathrm{~B} 30440005 \mathrm{E} 6 \mathrm{C}$ 40005E70 & $4 \longdiv { 0 0 5 E 7 4 }$ & $40005 \mathrm{E} 78$ 40005E $7 \mathrm{C}$ 40005F0C $40005 \mathrm{~F} 10$ \\
\hline a / $/ 13 . \operatorname{cpu}[0] . \mathrm{u} 0 / 1 \mathrm{eon} 3 \mathbf{x} 0 / \mathrm{p} 0 / \mathrm{iu} / \mathrm{r}$. d. pc & 40005 & 40005 & $0004 \mathrm{~B} 2 \mathrm{C} \times 40004 \mathrm{~B} 30 \times 40005 \mathrm{E} 6 \mathrm{C} X$ & $40005 E 70$ & 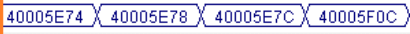 \\
\hline a- /13. cpu[0]. u0/1eon $3 \times 0 / p 0 / i u / d e \_s h a d o r$ & 40005 & 40005 & $0004 \mathrm{~B} 2 \mathrm{C} \times 40004 \mathrm{~B} 30 \times 40005 \mathrm{E} 6 \mathrm{C} X$ & $4 \longdiv { 0 0 5 E 7 0 }$ & 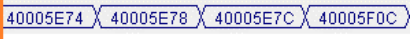 \\
\hline o- $/ 13 . \operatorname{cpu}[0] . \mathrm{u} 0 / 1 \mathrm{len} 3 \mathbf{x} 0 / \mathrm{p} 0 / \mathrm{iu} / \mathbf{r} \cdot \mathrm{a} \cdot \operatorname{ctr} 1 . \mathrm{pc}$ & 40005 & 40005 & $0004 \mathrm{~B} 284000 \ldots \times 4000 \ldots$ & $40,05 \mathrm{E} 6 \mathrm{C}$ & $40005 \mathrm{E} 7044000 \ldots \times 40004$ \\
\hline 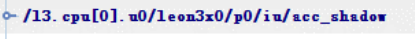 & 40005 & 40005 & $5004 \mathrm{~B} 28 \times 40004 \mathrm{~B} 2 \mathrm{C} \times 40004 \mathrm{~B} 30$ & $4005 \mathrm{E} 6 \mathrm{C}$ & 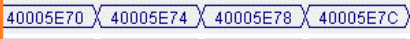 \\
\hline 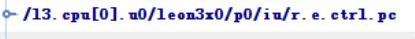 & 40005 & 40005 & $0004 \mathrm{~B} 24 \times 40004 \mathrm{~B} 28$ 40004B2C & $40004 \mathrm{~B} 30$ & $40005 \mathrm{E} 6 \mathrm{C} \times 40005 \mathrm{E} 70 \times 40005 \mathrm{E} 74 \times 40005 \mathrm{E} 78$ \\
\hline a- /13. epu[0]. u0/1eon $3 \times 0 / p 0 / i u / e_{-}$_shador & 40005 & 40005 & $5004 \mathrm{~B} 24 \times 40004 \mathrm{~B} 28$ 40004B2C & $43004 \mathrm{~B} 30$ & $40005 \mathrm{E} 6 \mathrm{C}, 40005 \mathrm{E} 70 \times 40005 \mathrm{E} 74 \times 40005 \mathrm{E} 78$ \\
\hline a- $/ 13 \cdot \operatorname{cpu}[0] \cdot \mathrm{u} 0 / 1 \mathrm{len} 3 \mathbf{x} 0 / \mathrm{p} 0 / \mathrm{iu} / \mathrm{r} \cdot \mathrm{m} \cdot \operatorname{ctr} 1 . \mathrm{pc}$ & 40005 & $400055^{3}$ & 104B20 $40004 \mathrm{~B} 24$ 40004B28 $X$ & $40004 \mathrm{~B} 2 \mathrm{C}$ & $40004 \mathrm{~B} 30 \times 40005 \mathrm{E} 6 \mathrm{C}, 40005 \mathrm{E} 70 \times 40005 \mathrm{E} 74$ \\
\hline a- /13. epu[0]. u0/1eon $3 \times 0 / p 0 / i u / m e \_s h a d o r$ & 40005 & 40005 & 104B20 $\times 40004 \mathrm{~B} 24 \times 40004 \mathrm{~B} 28 \mathrm{X}$ & $40004 \mathrm{~B} 2 \mathrm{C}$ & $40004 \mathrm{~B} 30 \times 40005 \mathrm{E} 6 \mathrm{C} \times 40005 \mathrm{E} 70 \times 40005 \mathrm{E} 74$ \\
\hline a) $/ 13 \cdot \operatorname{cpu}[0] \cdot \mathrm{u} 0 / 1 \mathrm{len} 3 \mathbf{x} 0 / \mathrm{p} 0 / \mathrm{iu} / \mathbf{r} \cdot \mathbf{x} \cdot \operatorname{ctr} 1 . \mathrm{pc}$ & 40005 & 40005 & $40004 \mathrm{~B} 20$ & $40004 \mathrm{~B} 28$ & $304 \mathrm{~B} 2 \mathrm{C} \times 4000 \ldots$ \\
\hline o- /13. cpu[0]. u0/1eon3x $0 / p_{0} 0 / i z / x_{-}$shador & 40005 & 40005 & $40004 \mathrm{~B} 24 \mathrm{C}$ & $F F B 42740004 B 28$ & $40004 \mathrm{~B} 2 \mathrm{C} \times 40004 \mathrm{~B} 30 \times 40005 \mathrm{E} 6 \mathrm{C} \times 40005 \mathrm{E} 70$ \\
\hline
\end{tabular}

Figure 4.6: The waveform of the result

In Fig. 4.6, the No.3 circle shows the error injected to the shadow register of the X stage. The value of it is flipped from 0x40004B28 to 0XBFFFB4D7. Consequently, the error is detected by the sensor network, as shown in the No.1 circle, Error Sense is asserted. In the meantime, as shown in the No.2 circle, the holdn signal is pulled down because of the error. As a result, the processor is stalled for one extra clock cycle for recovering from the soft error. As shown in the No.4 frame, the processor takes one extra clock cycle to repeat the instruction.

The descriptions above are about the error injection and verification of one error. For random error injection tests, we inject 112 errors in total in three rounds of tests, with 21, 53 and 38 number of errors injected respectively. All the results are verified to be correct in these tests. 


\subsection{Chapter Summary}

In this chapter, we presented the design of one single sensor and the sensor network. Then we implement our sensor network both on the LEON2 and LEON3 processors. By utilizing the synchronization feature of the LEON processors, we implement the error recovery scheme on LEON processors. From both the theoretical and the experimental analysis, we verified that the sensor network and the error recovery scheme can correctly detect the soft error and recover from it on the subsequent clock cycle. 



\section{Chapter 5}

\section{Overhead Evaluation}

In this chapter, we present our approach for evaluating the overheads of our rollback scheme. The overheads of a fault tolerant scheme include performance penalty and resource overhead.

The resource overhead usually has two kinds of impact: the increase of the die size and the decrease of the maximum clock frequency. The increase of the die size is because the error detection and recovery feature increases the number of logic gates. The decrease of the clock frequency is also caused by the extra logic gates. For an FPGA, more logic gates means greater depth of the clock distribution tree which implies lower maximum clock frequency.

As for the performance penalty, the extra error detection and rollback function could increase the length of the critical path and slow down the execution of applications. Moreover, after a soft error is detected, the rollback scheme takes time to recover from an error.

Therefore, we need to evaluate the penalty from these two impacts.

\subsection{Performance Penalty}

Recall that the pipeline rollback scheme is based on a synchronization feature of SPARC V8 structure. The feature aims to synchronize between FPU, IU, and CACHE by using a signal called holdn. The holdn signal can be asserted by the instruction cache (Icache), the data cache (Dcache) and FPU. When it is 
asserted by any of these components indicating that the component is not ready, IU would wait until all components release the holdn signal. The holdn signal does not hold back the execution of the other functional units but it does inform others that IU is stalled currently. Hence, if Icache senses that IU is stalled by the others, it could take this idle moment to do some work of its own, like refreshing the instruction cache.

Theoretically, if a soft error is detected by any sensors, the rollback scheme will stall IU from making further progress and make the pipeline re-execute the current instruction in the next clock cycle. This means, the rollback scheme usually incurs the penalty of one extra clock cycle. However, we found that the holdn signal exploited by our rollback scheme could cause Icache to refresh, which, in turn, stalls the processor for more than one cycle. Therefore, we need to find out the actual performance penalty of our rollback scheme.

\subsubsection{The Measurement Method of Performance Over- head}

In order to determine the performance penalty of the rollback scheme, we need to collect enough rollback timing data and derive the penalty caused by each error. In this way, we can get the statistic of the performance penalty.

To collect the rollback time, we need to inject errors during the execution of a test application. In our tests, COREMARK is the chosen application. In order to simulate more realistic scenarios, the errors are injected randomly. Moreover, the test cases include different error densities. That is, during the execution of the application, the number of errors injected are adjusted.

We design a test platform to carry out the experiment automatically. As illustrated in Fig. 5.1, we implement two LEON3 cores in an FPGA. Both cores have their own cache and internal resources, and they share AHB bus to access the external peripherals such as UART.

Core0 acts as a master which controls the test steps, generates signals for error injection and sends the test result back to a PC. Core1 is a test-target core which executes the COREMARK benchmark of EEMBC. In between, there is an error pulse generator module. This module is implemented by VHDL and is 


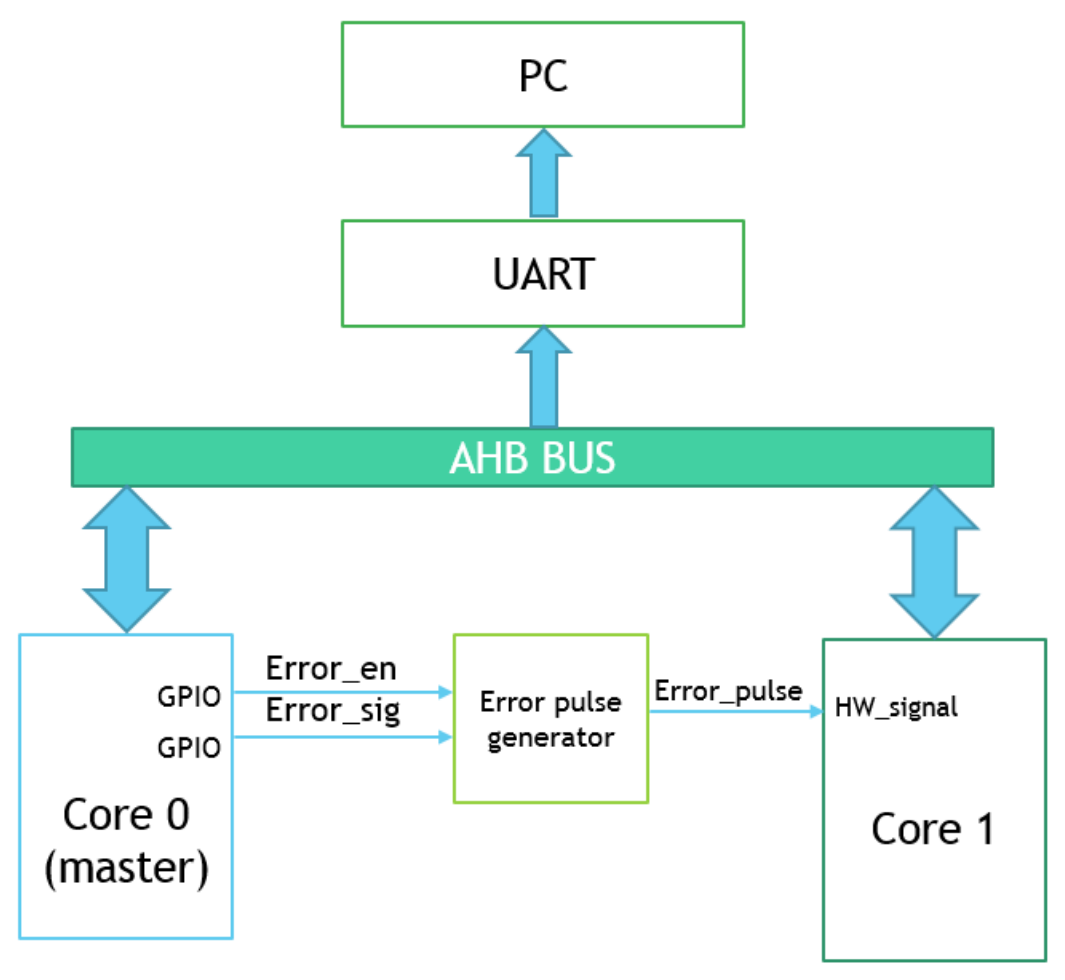

Figure 5.1: The test scheme

triggered by Core0. It generates a pulse of one clock cycle width, which is used to trigger the error circuit in Core1.

To control the dual-core platform, an operating system is instrumented. The operating system for multiprocessing can be divided into two different categories: asymmetric multiprocessing (AMP) and symmetric multiprocessing (SMP). The AMP system can assign different tasks to individual core whereas the SMP system distributes the tasks on all cores. In our case, Core0 needs to run an algorithm to test Core1, while Core1 is running the benchmark. Therefore, only the AMP system meets our requirements.

The Real-Time Executive for Multiprocessor Systems (RTEMS) is an open source Real Time Operating System (RTOS) which is compatible with the SPARC V8 architecture. Moreover, RTEMS could manipulate two cores separately which also meets our requirement. As shown in Fig. 5.1, in RTEMS, the two cores use semaphores to synchronize with each other. Core0 uses GPIO to control the er- 
ror generator module. Moreover, both cores transfer data to a PC through the UART port.

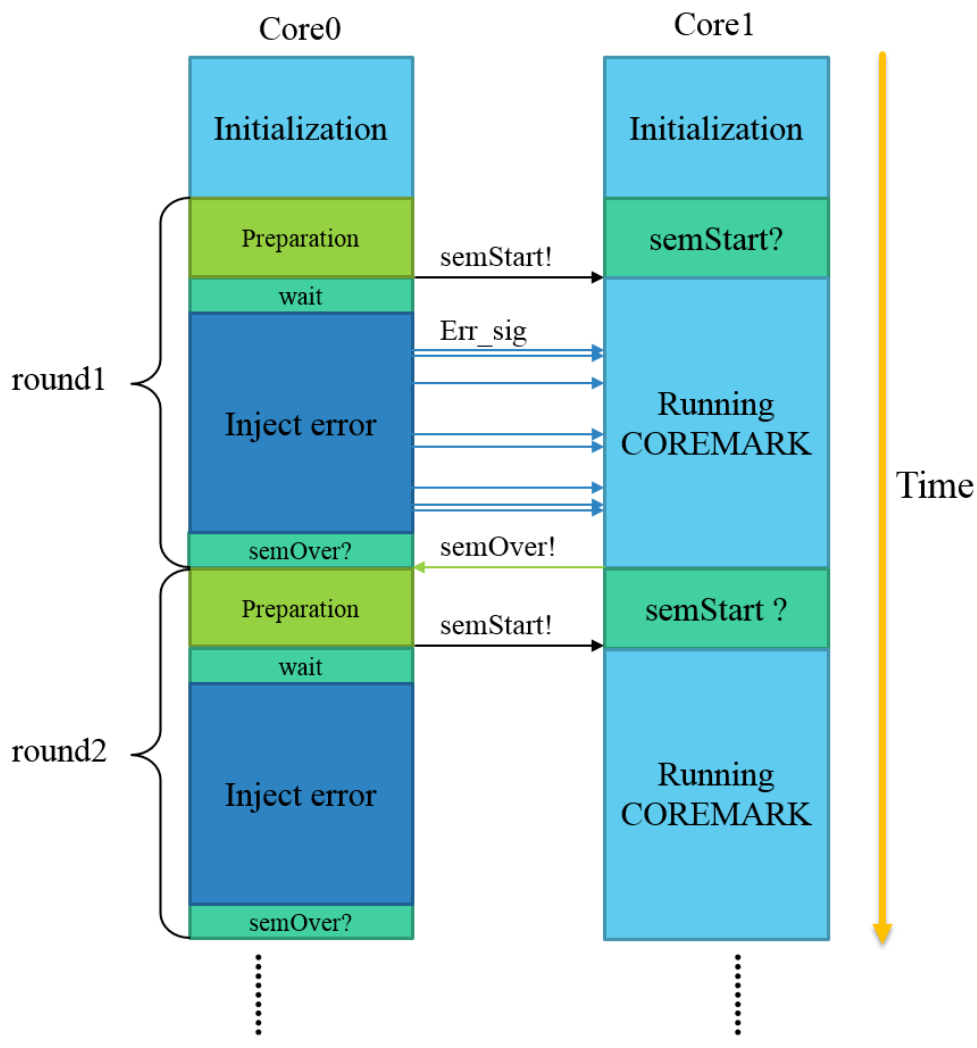

Figure 5.2: The Measurement flow

\subsubsection{Measurement Flow}

Fig. 5.2 shows the detailed test flow which contained six steps:

1. Both cores perform the same initialization of RTEMS at the beginning.

2. After the initialization, Core0 configures the test process according to the user's parameters. The parameters include the number of errors to be injected within one round of test, and the number of the test rounds to be carried out. In the meantime, Core1 waits for the Start signal. 
3. When the configuration of Core0 is over, it sends the Start signal to Core1. Consequently, Core1 starts to execute the COREMARK application. During the execution of COREMARK, Core0 randomly triggers the error injection pulse in order to inject errors to Core1.

4. At the end of COREMARK, Core1 reads the error information register, which is created by us and embedded into the hardware of the LEON3 processor in order to get the error ID and the performance penalty data, and then sends them to a PC.

5. After the execution of COREMARK, Core1 sends the End signal to Core0. When Core0 receives the End signal which indicates that one round of test is over.

6. Repeating the steps above till all required rounds of the tests are completed.

The number of error injections is used to create different test cases with different densities of errors. It is because we assume that a higher density of errors could cause more performance penalty. In our experiment, there are ten levels of error densities. The number of error injections are from 10, 20 times to 100 times during the same execution of the COREMARK application. Moreover, to guarantee enough samples for each test case, 1000 samples are collected in each case. For example, in a 10-error case, 10 number of errors are injected into one execution of the COREMARK. Since the total number of samples is 1000, it takes 100 rounds of test to get the 1000 samples.

In order to make sure that the density of errors injected are not clustered in time, we divide the execution time of the COREMARK into $N$ portions where $N$ is the number of error to be injected. Then in each portion, inject error to a random time point.

Before carrying out the tests, we need to get the execution time of the COREMARK first, and calculate the interval value $I$ according to the $N$ times of errors injected. The expression is shown in Eq. 5.1.

$$
I=\frac{\text { COREMARK Execution Time }}{N}
$$


For example, in the 10-error case, the execution time of the COREMARK is 76s. According to Eq. 5.1, the time interval for each error injection is $76 \mathrm{~s} / 10=$ 7.6s. That means an error should be injected randomly at any point of time during the 7.6s. And this process should be executed for 10 times to fulfill one round of test, and this test should be repeated for 100 times to get 1000 samples of the case.

\subsubsection{Overall Setups for Performance Overhead Measure- ments}

Fig. 5.3 shows the process flow chart of Core0.

There are two loops in this test flow. The first one is to count the number of the test rounds. One round of a test means one time execution of COREMARK on Core1. In other words, for Core0, one round of test indicates the time point from the sending of the Start signal to receiving the End signal. The second loop is to inject a specified number of errors.

For each time interval, an error is injected at a random point of it. However, getting a random number is not straightforward for embedded system. So, we use MATLAB to generate a list of 1000 random numbers ranging between 0 and 1. These random numbers are stored as an array in the C code of Core0's application. Moreover, we can choose the different entry point of this array to avoid getting the same pattern of the random number in each test case.

For an error injection, using $I$ to denote the time interval and $R$ to represent the random number, Core0 waits for $(I * R)$ seconds and controls GPIO to trigger the error injection module to inject an error to Core1. Then Core0 waits again for $(I *(1-R))$ seconds. This process is repeated to inject the next error. For Core1, the test flow is simple. As shown in Fig. 5.4, Core1 waits for the Start signal to execute the COREMARK application. At the end of COREMARK, it prints out the execution result along with the error information to a PC. Finally, it sends the End signal to Core0, then waits for the next round of execution on receiving the Start signal.

Fig. 5.5 is a snapshot of the GRMON command console. 


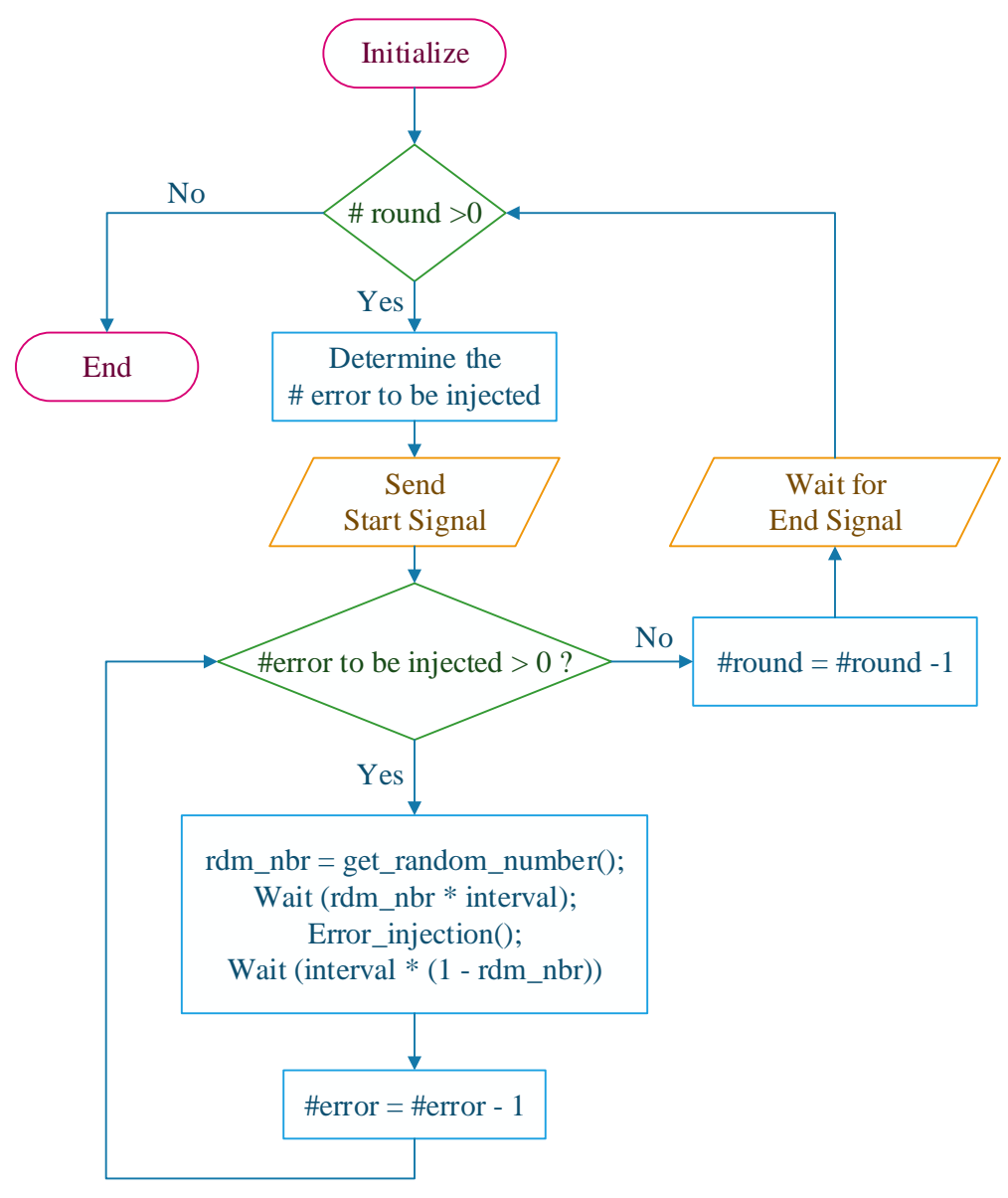

Figure 5.3: Core0 process flow chart

GRMON is a debugging tool of the LEON3 processor. It can check the configuration of a processor, view values of internal registers, download applications to a processor, etc.

As shown in Fig. 5.5, two applications are downloaded into two cores respectively. These applications are the images of RTEMS. The application of Core0, which includes the algorithm of the test, is indicated as Node1 in Fig. 5.5. The Core1's application is COREMARK and this application is indicated as Node2 in Fig. 5.5. And then configure the entry point and stack for each application. Finally, with the command "run", the two cores start to execute the applications together. 


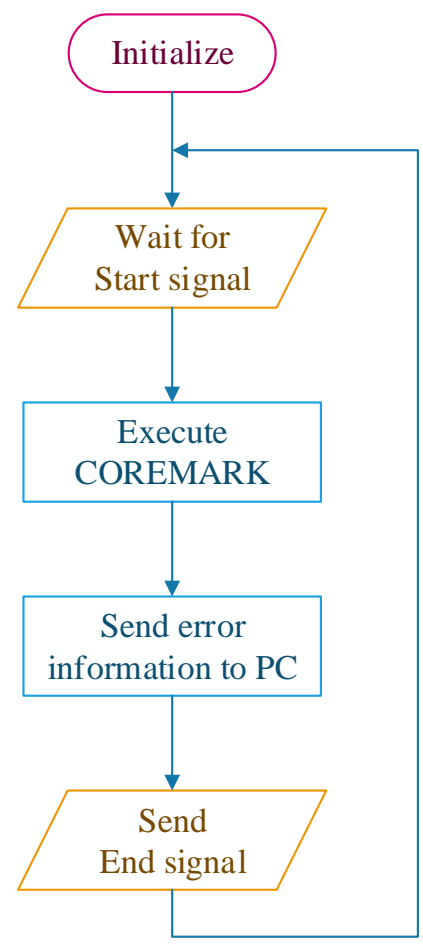

Figure 5.4: Core1 process flow chart

Fig. 5.6 is a snapshot of the UART console which displays one fraction of a test result. The frame No.1 indicates that currently the system is carrying out the 90 -error case, and the following content is the part of the 22nd round of the test.

The content between the frame No.1 and the frame No.2 is the execution result of COREMARK. The result shows that the execution time is $77.26 \mathrm{~s}$ with 3000 iterations, no data error and other detailed information of the benchmark.

The frame No.2 shows the current accumulated information of error register. According to the data, in the 22nd round of test, the accumulated number of errors is 90 and its corresponding accumulated performance penalty of these errors is 47 cycles. Since the data is accumulated, the actual data of the $22 \mathrm{nd}$ round is the difference between the 22 nd and the 21 st round of test.

The frame No.3 indicates that the 23rd round of test is beginning and the 


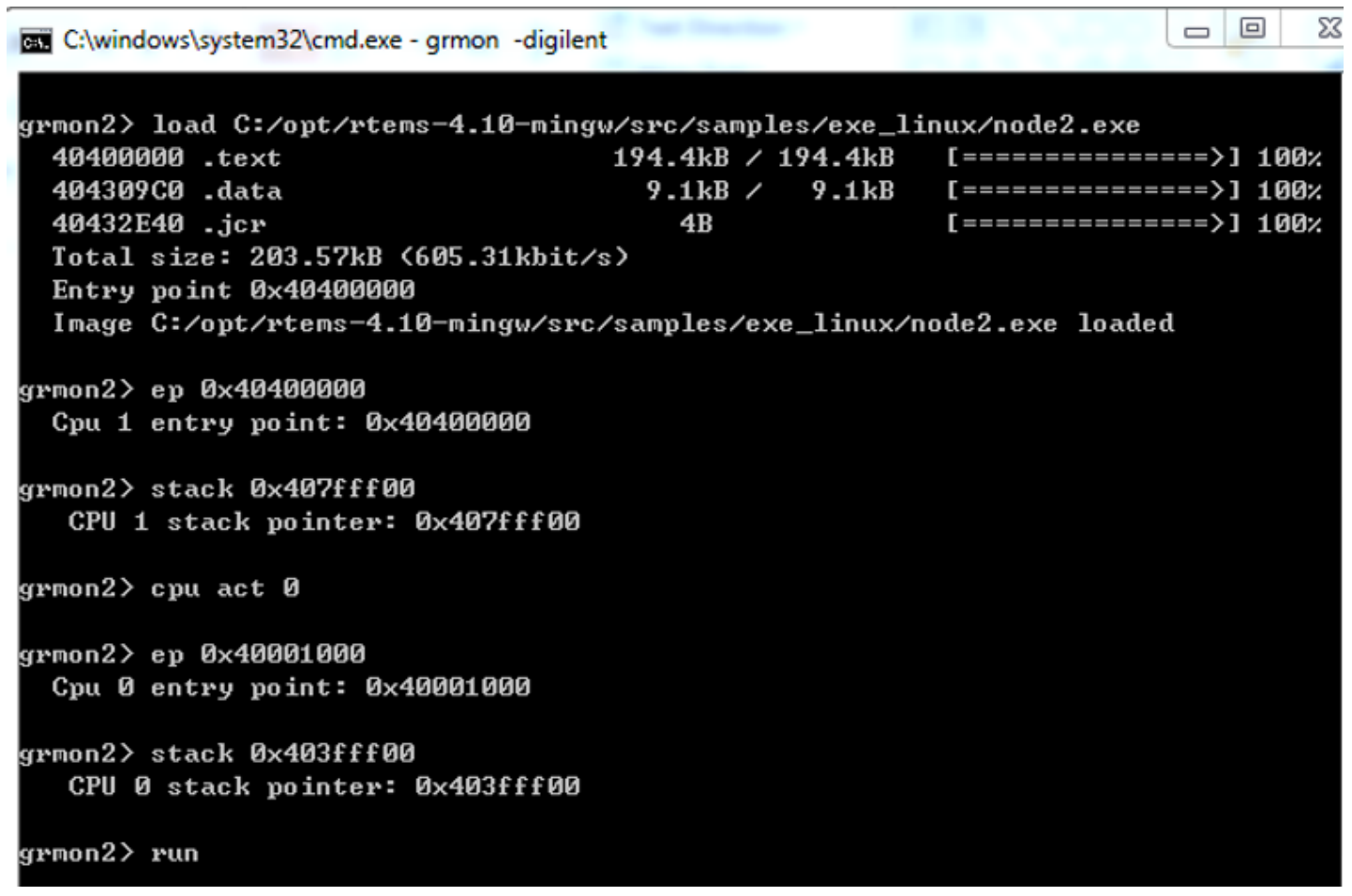

Figure 5.5: GRMON command snapshot

content below is about this particular round.

\subsubsection{Results of Performance Penalty Measurement}

Table 5.1 shows the final test result of the performance penalty. Each case has 1000 times of error injection. The error density is from 10 times of error injected to 100 times of error injected in the same execution of the COREMARK application.

Table 5.1 shows that between $86.2 \%$ and $89.9 \%$ of all cases, an error incurs one clock cycle of penalty. Between $9.50 \%$ and $12.5 \%$ of the cases, the penalty is 0 . In other cases, between $0.209 \%$ and $1.80 \%$, the penalty is greater than 1 . The number of such cases is negligible among the entire tests.

Fig. 5.7 shows the performance penalty distribution. Almost $90 \%$ of the errors cause the penalty of one clock cycle, no matter how intensive the error injection is. Less than $1 \%$ of errors cause more than one clock cycle penalty. About $10 \%$ of 


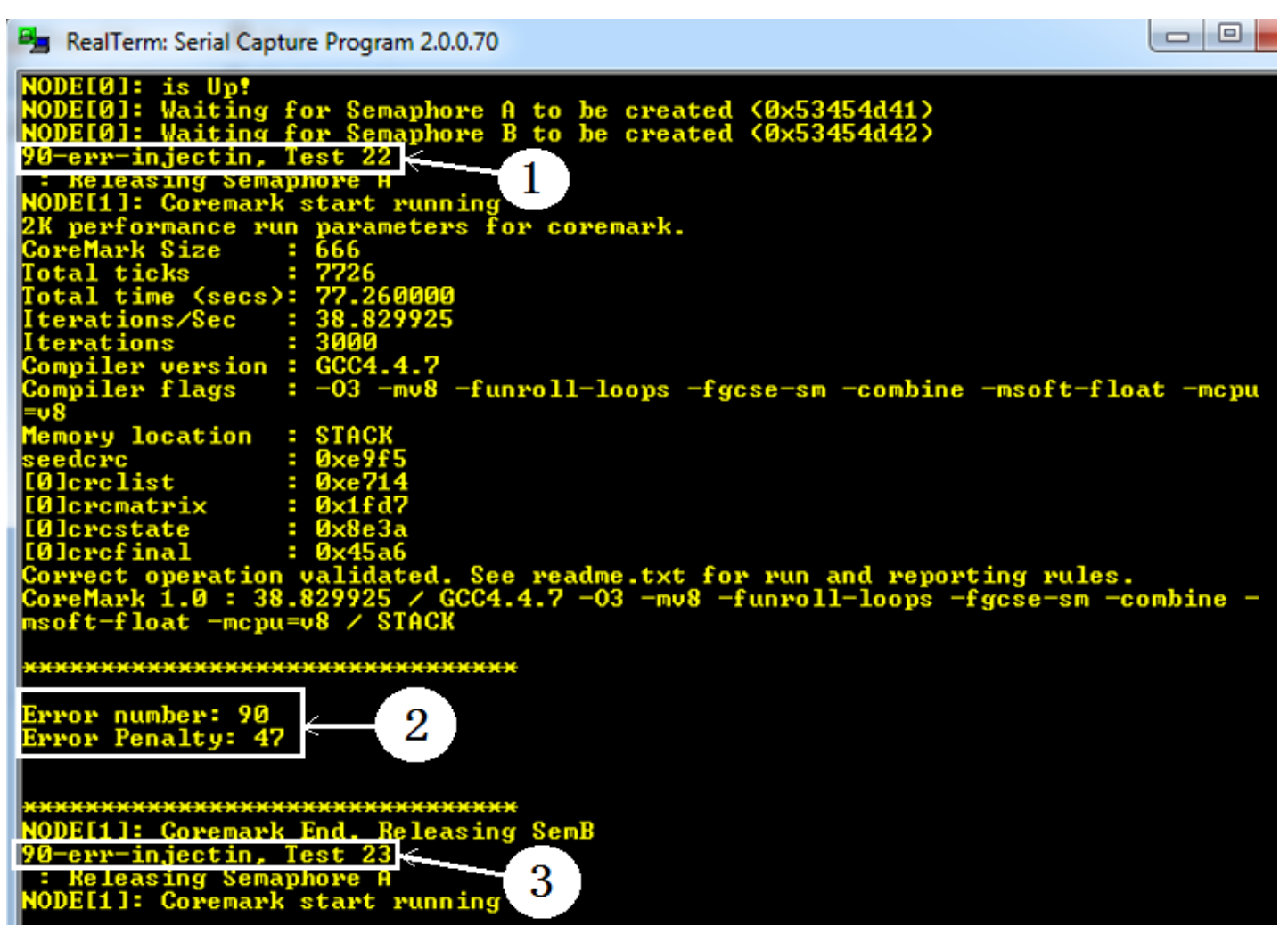

Figure 5.6: UART console snapshot

Table 5.1: Time penalty of all test case

\begin{tabular}{cccc}
\hline Casees & \% of 1 CC* & \% of 0 CC* & Others \\
\hline 10-err & $86.20 \%$ & $12.00 \%$ & $1.800 \%$ \\
20 -err & $88.00 \%$ & $11.10 \%$ & $0.900 \%$ \\
30 -err & $87.50 \%$ & $11.70 \%$ & $0.800 \%$ \\
40 -err & $89.80 \%$ & $9.50 \%$ & $0.700 \%$ \\
50 -err & $89.90 \%$ & $9.90 \%$ & $0.200 \%$ \\
60 -err & $88.63 \%$ & $11.16 \%$ & $0.209 \%$ \\
70 -err & $87.00 \%$ & $12.70 \%$ & $0.300 \%$ \\
80 -err & $88.11 \%$ & $11.37 \%$ & $0.521 \%$ \\
90 -err & $89.00 \%$ & $10.30 \%$ & $0.700 \%$ \\
100 -err & $87.20 \%$ & $12.50 \%$ & $0.300 \%$ \\
\hline
\end{tabular}

$* \mathrm{CC}$ is the abbreviation of Clock Cycle

the errors cause no performance penalty, because these errors are injected at the time when IU is held by Icache or Dcache, and during such time, error injection 
has no effect. Overall, we can draw a conclusion that our fault tolerant scheme incurs minimal performance penalties.

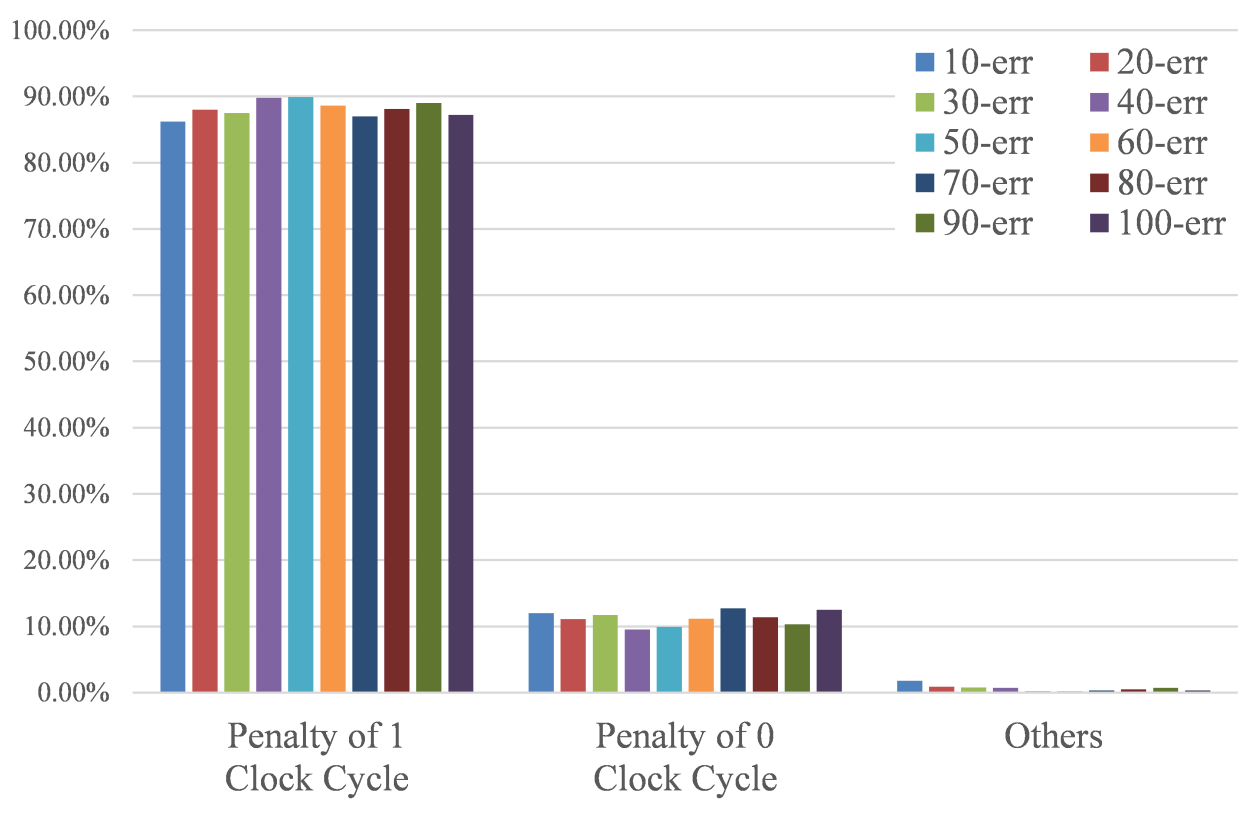

Figure 5.7: Time penalty distribution bar chart

\subsection{Resource Overhead Measurement}

The resource overhead is caused by the extra logic gates of the sensor network and the rollback scheme. The more sensors we add, the more logic gates are needed. Moreover, the extra logic gates also lengthen the critical path of the original logic of the processor. Therefore, the maximum clock frequency of a processor decreases because of the interference. Because of this, the extra resource usage and the decrease of the clock frequency should be determined in order to evaluate the performance of our rollback scheme.

Our current LEON3 processor and its fault tolerant scheme are implemented on an FPGA board. The development tool of FPGA could provide detailed report about the resource usage, estimated maximum clock frequency, and other useful information. We use the FPGA development tool to compare the difference 
between the processor with the fault tolerant scheme and the original processor, then analyze the different scenarios to obtain the resource overhead of our fault tolerant scheme.

\subsubsection{Experiment Environment}

In this experiment, Xilinx VC707 development board is the experimental platform. The sensor is added to the program counter (PC) of each stage of the LEON3 processor. Six 32-bit sensors are added one by one. After synthesis and implementation, we check the resource usage and the maximum frequency in the report of the development tool, which is Xilinx Vivado 2015.4.

The configuration of the LEON3 processor is:

- Library: grlib-gpl-1.4.1-b4156

- One SPARC V8 LEON3 core running @100Mhz

- Debug support unit (DSU) is included

- 2 sets $16 \mathrm{~KB}$ Icache and Dcache

- Memory management unit (MMU) is enabled

\subsubsection{Results of Resource Overhead Measurements}

Fig. 5.8 shows the total resource usage. The vertical axis indicates the resource usage of the FPGA, and the horizontal axis shows the number of 32-bit sensors.

It is noticed that the resource usage of adding the first sensor is more than adding the second and the other sensors. This is because, to add a sensor, the rollback scheme, and the corresponding verification logic have to be added together. Otherwise, the development tool will "optimize away" (eliminate) the sensors and rollback scheme as redundant logic, because the sensors and the logic cannot be activated if there is no trigger. In reality, the trigger is a flip of a register/bit caused by high energy particles. In the current design, the trigger is simulated by the hardware logic which is a part of the verification logic. So it 


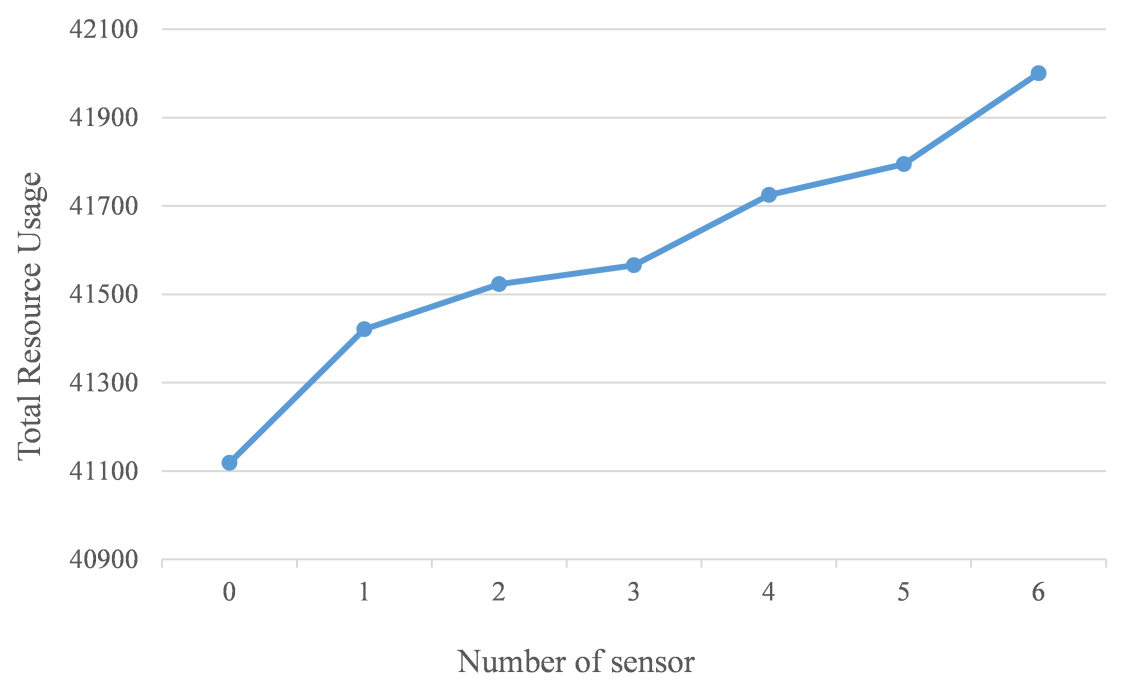

Figure 5.8: The total resource usage

has to be added to prevent the fault tolerant scheme from being optimized away. Therefore, the resource usage is different when adding the first sensor.

For the same reason, as shown in Fig. 5.9, when adding the first sensor, the maximum clock frequency, and the power usage also change more drastically than the other cases.

However, from both Fig. 5.8 and Fig. 5.9, after adding the first sensor, the resource usage, maximum clock frequency and the power usage of the subsequent sensors are not changed dramatically.

Table 5.2 suggests the percentage of the increase or decrease of the resource usage by comparing the original resource usage with that after adding sensors.

From Table 5.2, for adding one sensor, the processor takes $0.282 \%$ more logic cell on average and the maximum clock frequency is reduced by about $3.94 \%$ on average. The dynamic power consumption seems to keep constant when adding sensors. The reason is that the power is estimated by the Xilinx VIVADO tool and it is not the precise value. Since we do not trigger the error, the tool detects that all error detection circuits are idle and the dynamic power consumption of them are omitted. One solution of detecting the more precise power consumption is to run an application on a simulation. However this solution will cost lots of time, so we could do it in the future. 


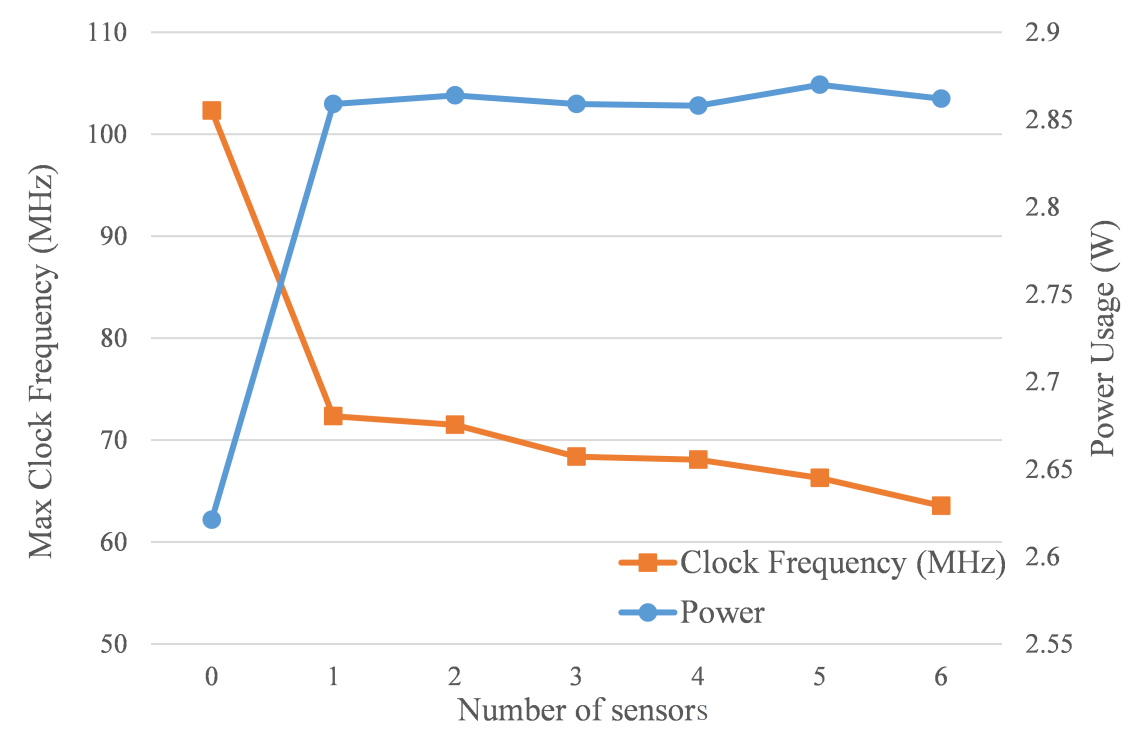

Figure 5.9: The maximum clock frequency and power usage

Table 5.2: Resource Usage

\begin{tabular}{lcccccc}
\hline Number of Sensors & 1 & 2 & 3 & 4 & 5 & 6 \\
\hline \hline Inc. of Logic Cell & $0.73 \%$ & $0.98 \%$ & $1.09 \%$ & $1.47 \%$ & $1.64 \%$ & $2.14 \%$ \\
\hline Dec. of Clock Freq. & $41.40 \%$ & $43.08 \%$ & $49.65 \%$ & $50.30 \%$ & $54.34 \%$ & $61.00 \%$ \\
\hline Inc. of Dynamic Power & $9.99 \%$ & $10.12 \%$ & $9.95 \%$ & $9.99 \%$ & $10.67 \%$ & $10.12 \%$ \\
\hline Inc. of Static Power & $0.77 \%$ & $0.77 \%$ & $0.77 \%$ & $0.77 \%$ & $0.77 \%$ & $0.77 \%$ \\
\hline
\end{tabular}

\subsection{Chapter Summary}

In this chapter, we described the overheads of our fault tolerant scheme from performance penalty and resource overhead.

The performance penalty is the main overhead of our scheme, which is required to recover the processor from a soft error. According to the experiments, about $90 \%$ of the test cases only incur one clock cycle of the performance penalty.

The resource overhead measures the extra logic cells used. Moreover, it also leads to the extra penalties, such as reduced maximum clock frequency and increased power consumption.

From the experimental data, the average cost of the logic cells for adding one 32 -bit sensor is about $0.282 \%$ of the original processor. 
For adding one sensor (32-bit), the reduction of the maximum clock frequency is about $3.94 \%$ on average. 



\section{Chapter 6}

\section{Identification of Critical Resources}

\subsection{Introduction}

To deal with soft errors, the ideal fault tolerant solution is to protect all registers and status bits of a computer system. However, for embedded systems, the hardware resource is limited. So it is impossible to protect all registers and bits. Besides, adding fault tolerant design to a system incurs some overheads, such as performance penalty, extra power consumption, increased die size, and a longer design time [19].

Since no system is $100 \%$ fault tolerant, a target level of fault tolerance is essential to constrain the design of a system. The industry usually uses Mean Time Between Failures (MTBF) to measure the error tolerant level of a system. Two kinds of errors can lead to failures: silent data corruption (SDC) and detected but unrecoverable errors (DUE) [19].

Take IBM for example. Its Power4 processor-based system has 1000 years MTBF for SDC errors. The MTBF of a system crash caused by DUE errors is 25 years. For an application crash caused by DUE errors, the MTBF is 10 years [8].

Another commonly used standard metric is FIT (Failure In Time) which is inversely related to MTBF. One FIT stands for one failure error in a billion hours. 
Zero FIT means zero error rate and infinite MTBF. The industry prefers FIT to MTBF because FIT is additive and it is convenient for calculation [19].

According to Mukherjee et al. [19], FIT could be affected by several vulnerability factors (VF). In general, a vulnerability factor suggests the probability of an error which can cause a system failure. For example, if the VF of a status bit of a processor is 10\%, it means the system will have $10 \%$ chances of being crashed if this bit is flipped because of a soft error.

By adopting the concept of VF, we measure the likelihood of a system failure when a soft error occurs during the execution of an instruction. The impact factor (IF) of a register/bit is the likelihood of an instruction failure when this register/bit has an error. Thus, in this chapter, we will focus on how to determine the IF of each register and status bit, and using the IF to determine the critical resources of a processor. Critical resources are the registers/bits with IF higher than a certain threshold $x$. The value of $x$ is determined by the requirement of the fault tolerance level. In our design, we set $x=1$, meaning the critical resources are registers/bits whose IF equals to 1.

Based on IF, we can selectively choose the critical resources to protect within limited hardware resources.

\subsection{Computation of IF}

In this section, we describe the methodology of computing the basic IF and an improved method for calculating the IF.

\subsubsection{Impact Factor}

The definition of IF of a bit/register is the probability of having value changes in other registers/bits of a processor when the target register/bit flipped because of a soft error.

We assume that in a time unit, the probability of a flip for every register and status bit of a processor is the same. Therefore, the longer time this register/bit is referenced (in a read or write operation) by the other bits/registers, the higher chance it can get flipped which in turn affects the results. Let $T$ denote the total 
time period of an application, $t$ denote the time register $R$ referenced by others, the expression of IF is given by Eq. 6.1.

$$
I F(R)=\frac{t}{T}
$$

For a digital system, time is measured by clock cycles. Therefore, for Eq. 6.1, the reference time of $R$ equals to the reference clock cycles. The total time period of an application equals to its total number of execution clock cycles. Initially, we will assume that every instruction costs one clock cycle. (To deal with different number of instruction cycles, we will discuss in Section 6.2.2.) That is, the number of clock cycles equals to the number of instructions. Thus, Eq. 6.1 can be transformed to Eq. 6.2.

$$
I F(R)=\frac{\text { Number of Instructions that Refer to } \mathrm{R}}{\text { Total Number of Instructions in This Application }}
$$

For example, assume an application executes 1000 instructions. Since one instruction takes one clock cycle, the total time period of this application is 1000 clock cycles. For a register X, assume it is accessed by 600 instructions meaning 600 clock cycles. Assume 200 instructions access the status bit Y, and for a register Z, which is rarely used, only 10 instructions access it.

According to Eq. 6.2, the IF of X, Y, and Z are:

$$
\begin{aligned}
& I F(X)=\frac{600}{1000}=0.6 \\
& I F(Y)=\frac{200}{1000}=0.2 \\
& I F(Z)=\frac{10}{1000}=0.01
\end{aligned}
$$

In this simplified approach, we convert the time to the instruction count, which is easier to implement and requires no extra hardware resources.

We can reform Eq. 6.2 as follows:

- Let $f_{\text {inst }}$ represent the frequency of an instruction inst in a target application 
- Let $R_{\text {inst }}$ represent the access condition of register/bit $R$ under the instruction inst. If inst accesses register/bit $R, R_{\text {inst }}=1$; otherwise, $R_{\text {inst }}=0$

- Let $n$ represent the number of different kinds of instructions executed in the target application

Then Eq. 6.2 can be simplified as follows:

$$
I F(R)=\sum_{i=1}^{n}\left(R_{i} * f_{i}\right)
$$

Using Eq. 6.4, there are two things to be determined, counting the instruction usage frequency and determining the specific registers/bits that every instruction is accessed.

\subsubsection{Weighted IF}

In the preceding section, we assume executing every instruction just takes one clock cycle. In fact, the number of cycles varies with different instructions. For LEON processors [13], the number of cycles of some instructions are listed in Table 6.1. We can use the numbers of cycles of an instruction as a weight to refine the calculation of the IF.

Table 6.1: Instruction timing [13]

\begin{tabular}{l|c}
\hline Instruction & Cycles(Weight) \\
\hline JMPL & 2 \\
Double Load & 2 \\
Single Store & 2 \\
Double Store & 2 \\
SMUL/UMUL & $1 / 2 / 4 / 35^{*}$ \\
SDIV/UDIV & 35 \\
Taken Trap & 4 \\
Atomic load/store & 3 \\
All Other Instruction & 1 \\
\hline
\end{tabular}

* depends on multiplier configuration 
Let $W_{\text {inst }}$ represent the weight of an instruction inst. Then Eq. 6.4 can be re-expressed as follows:

$$
I F(R)=\sum_{i=1}^{n}\left(R_{i} * f_{i} * W_{i}\right)
$$

According to Eq. 6.5, the IF of a register/bit under an instruction inst is determined by three factors: the access condition of a register $R_{\text {inst }}$, the frequency of instruction usage $f_{\text {inst }}$, and the weight of the instruction $W_{\text {inst }}$. The weight $W_{\text {inst }}$ is already determined in Table 6.1. The next sections describe how to get the other two factors.

\subsection{Instruction Frequency}

The instruction frequency and corresponding register/bit access depend on different applications. In this thesis, we use COREMARK as the test application. COREMARK is a benchmark from the Embedded Microprocessor Benchmark Consortium (EEMBC), which is used to evaluate the performance of embedded systems. It is written in $\mathrm{C}$ code and performs list processing, matrix manipulation, state machine and cyclic redundancy check (CRC) [30].

\subsubsection{Testing Plan}

There are two ways to get the instruction frequency: analyzing the program or adding hardware counters on hardware. For analyzing the program, there are two approaches. The first one is to (1) analyze the assembly code, and the another one is to (2) parse the execution history of instruction.

1. Assembly code is static code. What we can do is to analyze it by the keywords of an instruction. In this way, some iterated code cannot be counted. If the parameters of iterations are generated during run-time, in static analysis, the actual number of executed instructions cannot be determined, as the repeated code will be missed out. 
2. Analyzing the execution history. The LEON3 processor provides a debugger tool called GRMON which could trace and list out the recently executed instructions. The trace buffer of this feature is configurable up to $16 \mathrm{~KB}$. However, the records of one instruction require 64Byte, hence, $16 \mathrm{~KB}$ could store the history of at most 250 instructions, which is not big enough to store the entire COREMARK running history.

Therefore, both approaches are not applicable for COREMARK.

Another approach is to modify the hardware to add instruction counters in the decode stage to collect the statistic of instruction usage. Every instruction has its own 32-bit hardware counter. In order to check the values of these counters, the hardware debugging tool is an ideal choice.

\subsubsection{Hardware Debugging Tool}

The LEON3 processor is implemented on Xilinx Vertex-7 FPGA VC707 development board. Xilinx Vivado is the development tool. Vivado provides a debug IP which is similar to a logic analyzer which could be integrated with the target project to monitor the internal signals. With this built-in logic analyzer, we could customize the signals and registers to monitor, and get the result at the end of execution. The test flow is listed below:

1. To configure, add hardware counters and synthesize the LEON3 processor

2. To set up the debug core and add all counters in it

3. To synthesize again, start implementation and generate the Bitstream file

4. To download the Bitstream and debug probes file into FPGA

5. To execute COREMARK on the LEON3 processor and capture the values of all counters.

\subsubsection{The Measured Results of Instruction Frequency}

There are 114 kinds of instructions being monitored. The instruction usage statistics are shown in Table 6.2. 
Table 6.2: Instruction Usage Statistic

\begin{tabular}{|c|c|c|c|}
\hline Index & Instruction & Usage Times & Percentage \\
\hline 1 & mulscc & 140156928 & $17.206 \%$ \\
\hline 2 & bicc & 106422272 & $13.064 \%$ \\
\hline 3 & subcc & 63471616 & $7.792 \%$ \\
\hline 4 & add & 57880576 & $7.105 \%$ \\
\hline 5 & ld & 55152640 & $6.770 \%$ \\
\hline 6 & mov & 53827584 & $6.608 \%$ \\
\hline 7 & andcc & 45494272 & $5.585 \%$ \\
\hline 8 & i_and & 40220672 & $4.937 \%$ \\
\hline 9 & i_or & 28837888 & $3.540 \%$ \\
\hline 10 & i_sll & 25536512 & $3.135 \%$ \\
\hline 11 & i_srl & 21650432 & $2.658 \%$ \\
\hline 12 & st & 17702912 & $2.173 \%$ \\
\hline 13 & ldsh & 16611328 & $2.039 \%$ \\
\hline 14 & i_xor & 16251904 & $1.995 \%$ \\
\hline 15 & call & 16237568 & $1.993 \%$ \\
\hline 16 & retl & 13939200 & $1.711 \%$ \\
\hline 17 & nop & 11072000 & $1.359 \%$ \\
\hline 18 & ldub & 9553920 & $1.173 \%$ \\
\hline 19 & andncc & 9396224 & $1.153 \%$ \\
\hline 20 & addx & 9324544 & $1.145 \%$ \\
\hline 21 & subx & 9233408 & $1.133 \%$ \\
\hline 22 & orcc & 8905216 & $1.093 \%$ \\
\hline 23 & i_sra & 7926528 & $0.973 \%$ \\
\hline 24 & sethi & 6363392 & $0.781 \%$ \\
\hline 25 & jmp & 4603904 & $0.565 \%$ \\
\hline 26 & sub & 4432128 & $0.544 \%$ \\
\hline 27 & save & 2645376 & $0.325 \%$ \\
\hline 28 & restore & 2645376 & $0.325 \%$ \\
\hline 29 & ret & 2617344 & $0.321 \%$ \\
\hline 30 & addcc & 2008768 & $0.247 \%$ \\
\hline 31 & lduh & 1559808 & $0.191 \%$ \\
\hline 32 & sth & 1430656 & $0.176 \%$ \\
\hline 33 & $\operatorname{clr}$ & 765504 & $0.094 \%$ \\
\hline 34 & jmpl & 319264 & $0.039 \%$ \\
\hline 35 & ldsb & 184024 & $0.023 \%$ \\
\hline 36 & stb & 93024 & $0.011 \%$ \\
\hline
\end{tabular}


Table 6.2 (cont'd)

\begin{tabular}{clrr}
\hline Index & Instruction & Usage Times & Percentage \\
\hline 37 & i_std & 58476 & $0.007 \%$ \\
38 & ldd & 56640 & $0.007 \%$ \\
39 & rett & 14013 & $0.002 \%$ \\
40 & andn & 120 & $0.000 \%$ \\
41 & clrb & 48 & $0.000 \%$ \\
42 & clrh & 7 & $0.000 \%$ \\
43 & ticc & 2 & $0.000 \%$ \\
44 & others & 0 & $0.000 \%$ \\
& In Total & 814604019 & $100.0 \%$ \\
\hline \hline
\end{tabular}

Table 6.2 shows that 43 out of 114 instructions are used in the COREMARK application. Other instructions, such as floating point unit (FPU), hardware multiply and division and debug related instructions are never used. One reason is due to the configuration. The LEON3 processor does not have an FPU, hardware multiplier and divider. Hence, the related instructions are not used. Another reason is that the debug related instructions are only used in debug mode. For example, the instruction which is used to modify the special register can never be used in an ordinary application.

Fig. 6.1 shows a mixed plot of the instruction usage. The x-axis represents the list of instructions. The line chart represents the accumulated ratio of instruction usage, and its corresponding axis is on the left. The bar graph shows the proportion of individual instruction usage whose values are indicated on the vertical axis on the right side.

Fig. 6.1 shows that the top 18 instructions account for $90.8 \%$ of the whole instruction usage, because COREMARK has many iterated operations. These iterated operations include many multiply, divide and conditional instructions. Therefore, the most-used instruction is multiplication-MULSCC. Moreover, the second-most-used instruction is BICC, which is a set of conditional instructions. Ranking third is SUBCC, which is subtraction. This is due to the non-availability of hardware multiplier and divider in the test configuration of the LEON3. The multiplication and division are simulated by software. Since subtraction is the basic operation of division, the instruction SUBCC is also used a lot. 


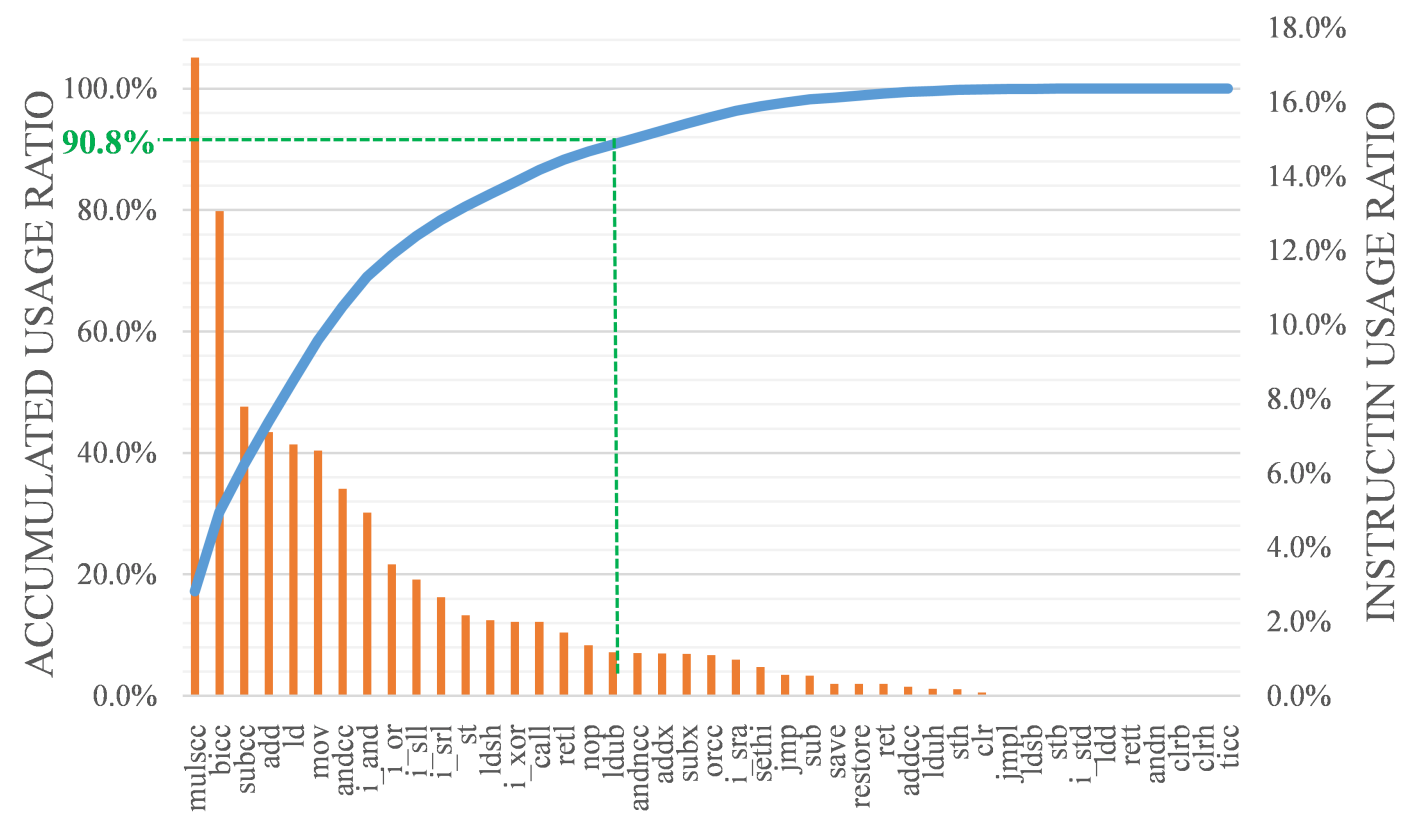

INSTRUCTINS

Figure 6.1: Instruction Usage Diagram

Since the top 18 instructions account for over $90 \%$ of the instruction usage, they can be considered as the most important instructions. And the remaining instructions are all considered as relatively unimportant. In impact factor analysis, these most important instructions will be used as samples to determine the critical resources.

\subsection{Access Frequencies of Register/Bit}

As we defined in the preceding section, the access of a register/bit means this register/bit is accessed (read or written) by other registers/bits in the processor. That means, if the value of an accessed register/bit changes, there must be some other value changes among all the registers/bits in the processor. 


\subsubsection{IF Measurements}

According to the definition above, for a given instruction, in order to check the IF of a register/bit, every register/bit needs to be flipped once, then check all the values of the other registers and bits.

For example, the LEON3 processor has a 7-stage pipeline, which includes Fetch (FE), Decode (DE), Register Access (ACC), Execution (EX), Memory (ME), Exception (X) and Write Back (WB) stage. The 7-stage pipeline consists of six middle-register sets, which are FE-DE, DE-ACC, ACC-EX, EX-ME, ME-X, $\mathrm{X}$-WB middle-register set.

When the LEON3 processor is executing a specific instruction, we flip a register/bit and check the value that may be changed in the six sets of middle-registers.

Table 6.3: Register/Bit Number

\begin{tabular}{lc}
\hline Name & Number of Reg/Bit \\
\hline FE-DE & 33 \\
DE-ACC & 44 \\
ACC-EX & 42 \\
EX-ME & 38 \\
ME-X & 37 \\
X-WB & 29 \\
\hline Total & 223 \\
\hline
\end{tabular}

Table 6.3 shows the number of registers/bits of each middle-stage contained. There are 223 registers and bits in total.

In theory, in order to determine the access of a register/bit, every register/bit needs to be flipped once. For each flip, all of the other registers and bits need to be examined. That is, for each instruction, we need to check $233^{2}=54289$ times in total.

For this many times of flipping and checking, using ModelSim simulation tool is an ideal solution. Because it is easy to automate the processes of flipping and checking of register values. 


\subsubsection{Test Flow}

First of all, we need to run a basic version of simulation with an example application to make sure the entire test process is correct. Then we add the instructions to be tested at the end of this example application and rerun it to carry out our test.

The tested instructions are decoupled in order to get an independent test result, because instructions in the pipeline can influence each other.

To achieve this, the target instruction is preceded by a sequence of NOP instructions. The pipeline is "cleaned up" by the NOP instructions which prepare the pipeline registers/bits in their default status.

For example, testing the access condition under the instruction ST (to store one byte of data to memory), six middle-register sets need to be tested one by one. In this example, the ACC-EX register set is the target set. The basic test flow is:

1. Execute 18 NOP instructions around the ST instruction to "clean up" the pipeline. Since the LEON3 processor has a 7-stage pipeline, theoretically, we can execute $7 \mathrm{NOP}$ instructions before the target instruction and 7 NOP instructions after the execution of target instruction for cleaning up the pipeline. For safety, we execute two more NOP instructions, that is 9 NOP instructions before and 9 after.

2. Execute the application once, get the address of the ST instruction.

3. Re-execute the application and monitor the program counter (PC) of ACC stage. Wait till the PC of the ACC stage points to the address of the ST instruction, then record all values of register as the initial value.

4. Iteratively flip every register in the ACC stage, and check the value after each flip. If there is any value change compared to the initial value, then recode the corresponding registers.

Above is the process to test one middle-register set. In our case, all the six middle-register sets need to be tested. So, we repeat the steps above for six times to finish the test. 


\subsubsection{Test Automation}

In theory, all instructions need to be tested. However, according to Table 6.2, only 43 kinds of instructions are used in COREMARK application. The top18 most frequently used instructions account for over $90 \%$ of the register usage. Therefore, it is reasonable to consider only these top-18 instructions in order to reduce the workload.

Although we reduce the number of the sample instructions to 18 , the total number of tests are still $18 * 233^{2}=977202$ times. This is a lot of work if we carry out it manually. Hence, an automation process is necessary.

ModelSim provides the Application Programming Interface (API) which can be utilized by Tcl (Tool Command Language) script. Tcl scripts are commonly used for rapid prototyping, scripted applications and, GUIs and testing [33]. By adopting Tcl scripts, we can easily perform repetitive tests.

Listing 6.1 is an example of an automation script. There are three main loops in this automated test flow. Line 1 is the main loop to go through all middleregister set of LEON3. Line 5 is the test loop to simulate the register flip for every register/bit in the target register set. Line 9 is the verification loop used to check all value of registers/bits in the processor and report the differences.

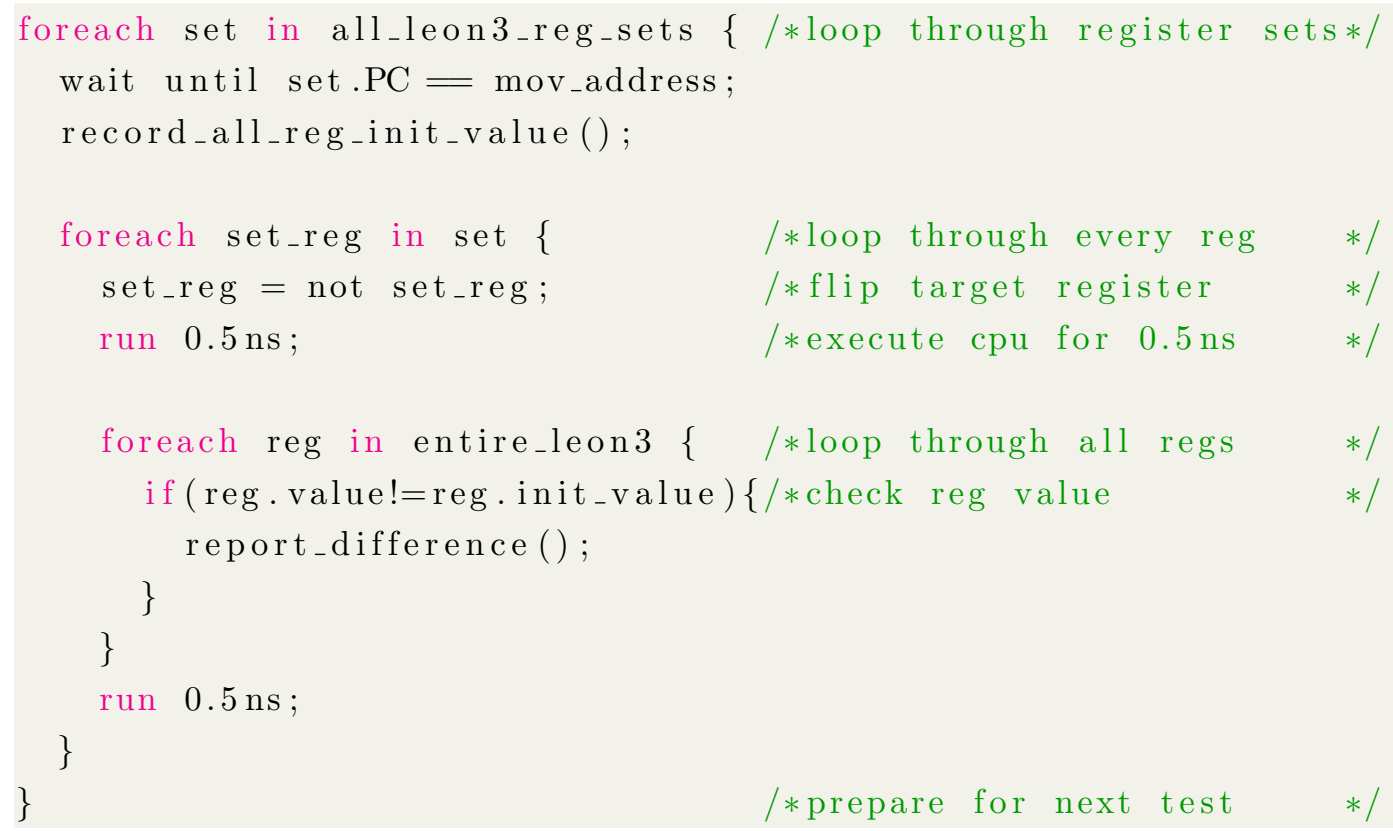

Listing 6.1: Automated Test Flow Script 
Fig. 6.2 shows the automated test flow chart. As mentioned in preceding section, the LEON3 processor has six sets of middle-registers. In the test flow, it goes through every set of the middle-register. For each register set, loop through each register/bit and flip it. For each register, check the value of every other register/bit and compare it with their initial values.

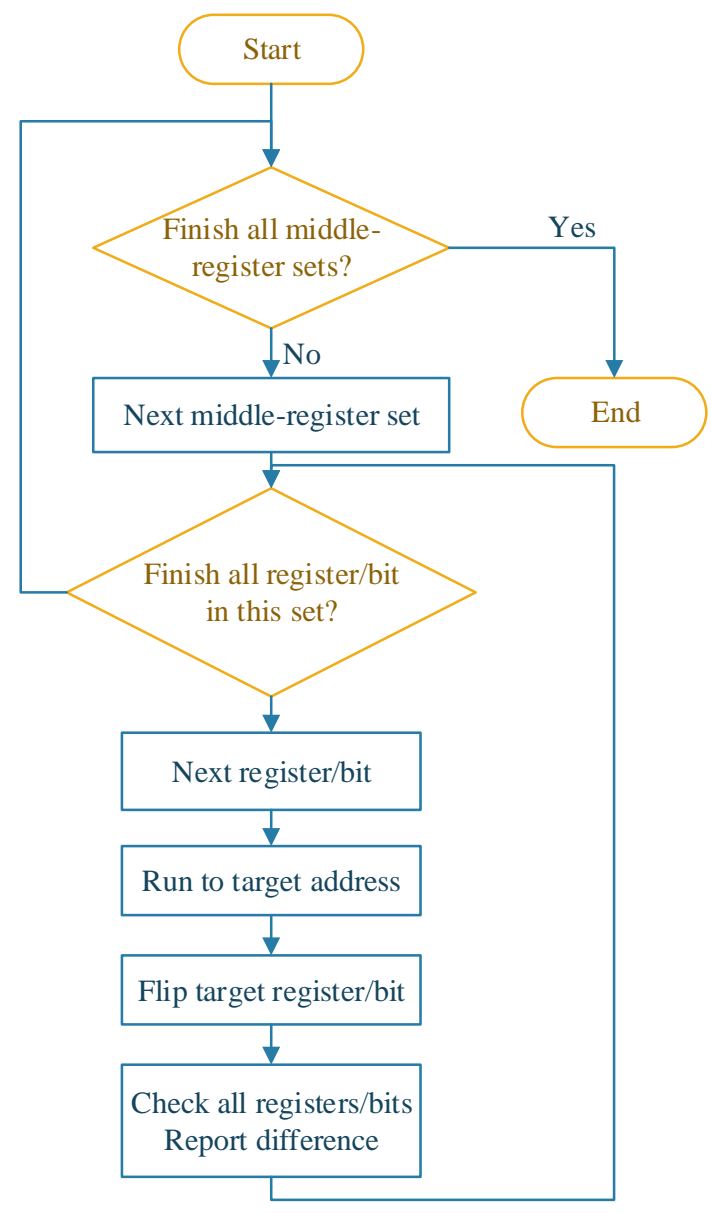

Figure 6.2: Automated Test Flow Chart

Fig. 6.3 shows a snapshot of testing the register set of the ACC-EX stage. The No.1 frame shows the part of signals in the ACC-EX stage. In the same row with the signal is its corresponding waveform. The spiked signals shown in the No.2 frame are the simulated flip in every register/bit in the ACC-EX stage. 
By flipping every register at different point in time, we check the value changes of other registers/bits to determine whether this bit is accessed or not.

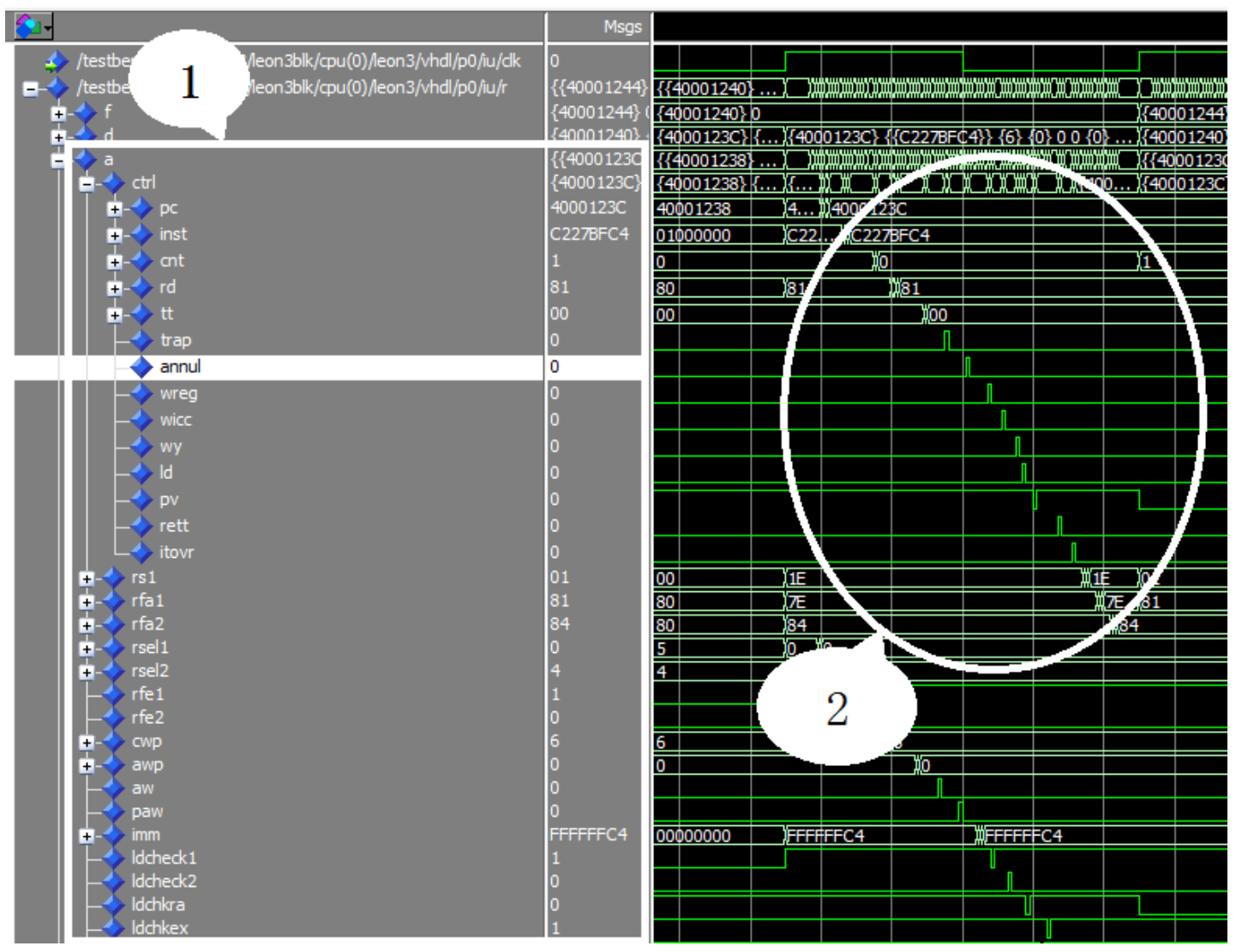

Figure 6.3: Automated Test Snapshot

\subsubsection{The Measured Results of Access of Register/Bit}

Table 6.4 shows a part of result of the access condition of the ACC stage. The detailed full result of all stages is in Table A.1 of Appendix A.

The first row is the top-18 instructions and the first column is the part of the registers of ACC stage. The little check marks indicate the access condition of the register under the corresponding instruction.

As the result shown in Table 6.4, not all registers/bits are used in the top-18 instruction of COREMARK application. For instance, a.ctrl.wy is accessed by 
all top-18 instructions. In contrast, the a.rs1 register is not accessed in all of the sample instructions. a.rfa1 and a.rfa2 are accessed by few instructions.

Table 6.4: The Part of Access Condition of ACC Stage Register/Bit

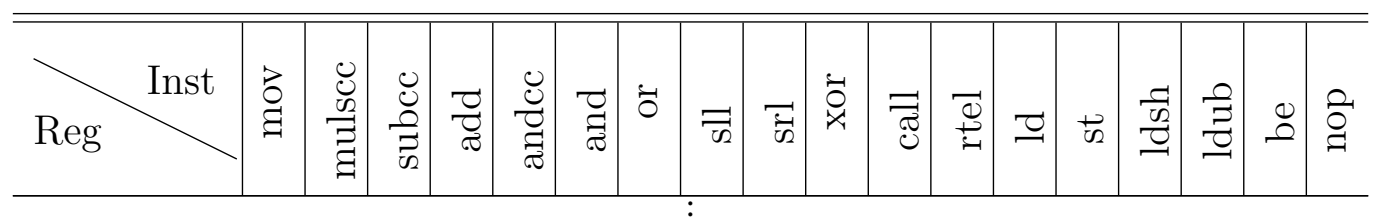

\begin{tabular}{|c|c|c|c|c|c|c|c|c|c|c|c|c|c|c|c|c|c|c|}
\hline a.ctrl.wy & $\checkmark$ & $\checkmark$ & $\checkmark$ & $\checkmark$ & $\checkmark$ & $\checkmark$ & $\checkmark$ & $\checkmark$ & $\checkmark$ & $\checkmark$ & $\checkmark$ & $\checkmark$ & $\checkmark$ & $\checkmark$ & $\checkmark$ & $\checkmark$ & $\checkmark$ & $\checkmark$ \\
\hline a.ctrl.ld & $\checkmark$ & $\checkmark$ & $\checkmark$ & $\checkmark$ & $\checkmark$ & $\checkmark$ & $\checkmark$ & $\checkmark$ & $\checkmark$ & $\checkmark$ & $\checkmark$ & $\checkmark$ & $\checkmark$ & $\checkmark$ & $\checkmark$ & $\checkmark$ & $\checkmark$ & $\checkmark$ \\
\hline a.ctrl.pv & $\checkmark$ & $r$ & $\checkmark$ & $\checkmark$ & $\checkmark$ & $\checkmark$ & $\checkmark$ & $\checkmark$ & $\checkmark$ & $\checkmark$ & $\checkmark$ & $\checkmark$ & $\checkmark$ & $\checkmark$ & $\checkmark$ & $\checkmark$ & $\checkmark$ & $\checkmark$ \\
\hline a.ctrl.rett & $\checkmark$ & $\checkmark$ & $\checkmark$ & $\checkmark$ & $\checkmark$ & $\checkmark$ & $\checkmark$ & $\checkmark$ & $\checkmark$ & $\checkmark$ & $\checkmark$ & $\checkmark$ & $\checkmark$ & $\checkmark$ & $\checkmark$ & $\checkmark$ & $\checkmark$ & $\checkmark$ \\
\hline a.ctrl.itovr & $\checkmark$ & $\checkmark$ & $\checkmark$ & $\checkmark$ & $\checkmark$ & $\checkmark$ & $\checkmark$ & $\checkmark$ & $\checkmark$ & $\checkmark$ & $\checkmark$ & $\checkmark$ & $\checkmark$ & $\checkmark$ & $\checkmark$ & $\checkmark$ & $\checkmark$ & $\checkmark$ \\
\hline \multicolumn{19}{|l|}{ a.rs1 } \\
\hline a.rfa1 & & $\checkmark$ & $\checkmark$ & & & & & & & $\checkmark$ & $\checkmark$ & & & & & & & \\
\hline a.rfa2 & & $\checkmark$ & $\checkmark$ & & & & & & & $\checkmark$ & $\checkmark$ & & & & & & & \\
\hline a.rsel1 & $\checkmark$ & $\checkmark$ & $\checkmark$ & $\checkmark$ & $\checkmark$ & $\checkmark$ & $\checkmark$ & $\checkmark$ & $\checkmark$ & $\checkmark$ & $\checkmark$ & $\checkmark$ & $\checkmark$ & $\checkmark$ & $\checkmark$ & $\checkmark$ & $\checkmark$ & $\checkmark$ \\
\hline a.rsel2 & $\checkmark$ & $\checkmark$ & $\checkmark$ & $\checkmark$ & $\checkmark$ & $\checkmark$ & $\checkmark$ & $\checkmark$ & $\checkmark$ & $\checkmark$ & $\checkmark$ & $\checkmark$ & $\checkmark$ & $\checkmark$ & $\checkmark$ & $\checkmark$ & $\checkmark$ & $\checkmark$ \\
\hline a.rfe1 & $\checkmark$ & $\checkmark$ & $\checkmark$ & $\checkmark$ & $\checkmark$ & $\checkmark$ & $\checkmark$ & $\checkmark$ & $\checkmark$ & $\checkmark$ & $\checkmark$ & $\checkmark$ & $\checkmark$ & $\checkmark$ & $\checkmark$ & $\checkmark$ & $\checkmark$ & $\checkmark$ \\
\hline a.rfe2 & $\checkmark$ & $\checkmark$ & $\checkmark$ & $\checkmark$ & $\checkmark$ & $\checkmark$ & $\checkmark$ & $\checkmark$ & $\checkmark$ & $\checkmark$ & $\checkmark$ & $\checkmark$ & $\checkmark$ & $\checkmark$ & $\checkmark$ & $\checkmark$ & $\checkmark$ & $\checkmark$ \\
\hline
\end{tabular}

\subsection{The Results of Critical Resource Measure- ment}

As described in Section 6.2.2, computation of IF requires three factors:

- the frequency of instruction usage

- the access condition of register/bit under specific instruction

- the weight of instruction

Since all required data are collected in the preceding Sections, we can utilize Eq. 6.5 to calculate the IF of each register. 
Chapter 6. Identification of Critical Resources

Table 6.5: IF of All Registers

\begin{tabular}{|c|c|c|c|c|c|}
\hline IF & \multicolumn{4}{|c|}{ Registers } & Quantity \\
\hline 1 & $\begin{array}{l}\text { d.pc } \\
\text { d.cnt } \\
\text { d.rexen } \\
\text { a.ctrl.cnt } \\
\text { a.ctrl.wicc } \\
\text { a.ctrl.rett } \\
\text { a.rfe1 } \\
\text { a.et } \\
\text { e.ctrl.pc } \\
\text { e.ctrl.tt } \\
\text { e.ctrl.wicc } \\
\text { e.ctrl.rett } \\
\text { e.ldbp1 } \\
\text { e.bp } \\
\text { m.ctrl.rd } \\
\text { m.ctrl.wreg } \\
\text { m.ctrl.pv } \\
\text { m.y } \\
\text { m.dci.read } \\
\text { m.dci.size } \\
\text { x.ctrl.pc } \\
\text { x.mexc } \\
\text { w.s.s }\end{array}$ & $\begin{array}{l}\text { d.inst }(0) \\
\text { d.pv } \\
\text { d.rexpos } \\
\text { a.ctrl.rd } \\
\text { a.ctrl.wy } \\
\text { a.ctrl.itovr } \\
\text { a.rfe2 } \\
\text { a.jmpl } \\
\text { e.ctrl.inst } \\
\text { e.ctrl.trap } \\
\text { e.ctrl.wy } \\
\text { e.ctrl.itovr } \\
\text { e.ldbp2 } \\
\text { m.ctrl.pc } \\
\text { m.ctrl.tt } \\
\text { m.ctrl.wicc } \\
\text { m.ctrl.rett } \\
\text { m.icc } \\
\text { m.dci.write } \\
\text { m.dci.asi } \\
\text { x.ctrl.rd } \\
\text { x.nerror } \\
\text { w.s.et }\end{array}$ & $\begin{array}{l}\text { d.cwp } \\
\text { d.annul } \\
\text { a.ctrl.pc } \\
\text { a.ctrl.annul } \\
\text { a.ctrl.ld } \\
\text { a.rsel1 } \\
\text { a.cwp } \\
\text { a.bp } \\
\text { e.ctrl.cnt } \\
\text { e.ctrl.annul } \\
\text { e.ctrl.ld } \\
\text { e.op1 } \\
\text { e.su } \\
\text { m.ctrl.inst } \\
\text { m.ctrl.trap } \\
\text { m.ctrl.wy } \\
\text { m.ctrl.itovr } \\
\text { m.dci.signed } \\
\text { m.dci.lock } \\
\text { m.mac } \\
\text { x.ctrl.trap } \\
\text { w.s.tt } \\
\text { w.s.y }\end{array}$ & $\begin{array}{l}\text { d.mexc } \\
\text { d.step } \\
\text { a.ctrl.inst } \\
\text { a.ctrl.wreg } \\
\text { a.ctrl.pv } \\
\text { a.rsel2 } \\
\text { a.su } \\
\text { a.bpimiss } \\
\text { e.ctrl.rd } \\
\text { e.ctrl.wreg } \\
\text { e.ctrl.pv } \\
\text { e.alucin } \\
\text { e.mac } \\
\text { m.ctrl.cnt } \\
\text { m.ctrl.annul } \\
\text { m.ctrl.ld } \\
\text { m.result } \\
\text { m.dci.enaddr } \\
\text { m.dci.dsuen } \\
\text { m.su } \\
\text { x.ctrl.ld } \\
\text { w.s.ef }\end{array}$ & 91 \\
\hline$[0.971,0.823]$ & $\begin{array}{l}\text { W.s.s } \\
\text { d.inull } \\
\text { e.alusel }\end{array}$ & $\begin{array}{l}\text { W.s.et } \\
\text { m.casa }\end{array}$ & $\begin{array}{l}\text { w.s.y } \\
\text { x.ctrl.annul }\end{array}$ & e.op2 & 5 \\
\hline$[0.786,0.711]$ & m.wcwp & w.s.icc & e.jmpl & & 3 \\
\hline$[0.65,0.632]$ & $\begin{array}{l}\text { w.s.ec } \\
\text { w.s.dbp } \\
\text { w.except } \\
\text { w.s.dwt } \\
\end{array}$ & $\begin{array}{l}\text { w.s.ps } \\
\text { w.s.dbprepl } \\
\text { w.twcwp } \\
\text { w.wreg }\end{array}$ & $\begin{array}{l}\text { w.s.asr18 } \\
\text { w.result } \\
\text { m.werr }\end{array}$ & $\begin{array}{l}\text { w.s.svt } \\
\text { w.wa } \\
\text { x.result }\end{array}$ & 14 \\
\hline$[0.553,0.500]$ & r.a.imm & r.w.s.pil & r.x.ctrl.wreg & r.x.intack & 4 \\
\hline$[0.473,0.368]$ & r.w.s.cwpmax & r.x.data $(0)$ & & & 2 \\
\hline$[0.266,0.214]$ & r.a.rfa1 & r.a.rfa2 & r.e.aluadd & & 3 \\
\hline$[0.173,0.115]$ & $\begin{array}{l}\text { r.e.aluop } \\
\text { r.x.ctrl.wicc }\end{array}$ & $\begin{array}{l}\text { r.w.s.wim } \\
\text { r.x.ctrl.wy }\end{array}$ & $\begin{array}{l}\text { r.e.ymsb } \\
\text { r.x.y } \\
\end{array}$ & $\begin{array}{l}\text { r.e.mulstep } \\
\text { r.x.icc }\end{array}$ & 8 \\
\hline$[0.077,0.027]$ & r.w.s.stwin & r.e.shcnt & r.m.nalign & r.e.shleft & 4 \\
\hline 0 & others & & & & 89 \\
\hline & & In Total & & & 223 \\
\hline
\end{tabular}


Table 6.5 is the final IF data of all registers and bits in LEON3 processor. Table 6.5 shows that 91 out of 223 registers and bits have IF equals to 1 , by our definition, they are critical resources. The IF of 89 registers/bits is 0 . This means they are not important for the COREMARK application. The IF of the remaining 43 registers/bits has values ranging between 0.027 to 0.97 , meaning they are less important registers/bits. Therefore, registers having the highest IF should be protected first if the resource is limited. By referring to Table 6.5, we can easily select the registers/bits for protection.

\subsection{Chapter Summary}

In this chapter, we try to identify the critical resources of LEON processors. First of all, we define the impact factor (IF) of a register/bit. That is when the target register/bit flipped because of a soft error, the probability of having value changes in other registers/bits of a processor. According to the definition of IF, there are three factors affects the IF: the access condition of a register/bit, the frequency of instruction usage and the weight of the instruction. Then we determine the three factors and calculate the IF of each register/bit accordingly. Finally, we find out that the IF of 89/223 (39.9\%) of registers/bits are 0, meaning they will not cause any trouble even if errors happen on them. The IF of 43/223 (19.3\%) of registers/bits has values ranging between 0,027 to 0.97 , meaning they are less important registers/bits. And the IF of the rest registers/bits (91/223, 40.8\%) are 1 , meaning they have first priority when choosing the protection registers/bits. 



\section{Chapter 7}

\section{Discussions and Conclusion}

In this chapter, we summarize the thesis work and discuss the future work.

\subsection{Summary and Results}

The main work of the thesis can be summarized as follows:

1. We implemented the fault tolerant scheme, i.e., the sensors network and the error recovery scheme on the LEON2 and LEON3 processors. According to the test results, the extra logic added to the processor does not influence the normal function of the processor.

2. We verified the correctness of the fault tolerant scheme by using two approaches. We injected errors during the execution of the individual instructions of LEON2 and checked the execution results and internal signals to verify their correctness. Moreover, we also inject random errors while the LEON2 processor is executing the COREMARK application, and verify it from the execution results and the internal waveforms. Both verifications suggest that the fault tolerant scheme work correctly on the LEON2 processor.

3. We migrated the fault tolerant scheme to an LEON3 processor and verified the correctness of the scheme by injecting random errors while the processor 
is executing the COREMARK application. The result of the verification shows that the fault tolerant scheme works correctly on the LEON3 processor.

4. We determined the overhead of the scheme from the performance penalty, the resource overhead, the power consumption, and the peak clock frequency. For performance penalty, the scheme only uses one clock cycle to recover from an error in about $90 \%$ of the test cases. For resource overhead, adding one sensor only costs $0.282 \%$ resource of the original processor. The power consumption increases by about $0.07 \%$ for adding one sensor. The peak clock frequency decreases by about $3.94 \%$ on average for adding one sensor.

5. We identified the most critical register/bit of the LEON3 processor by determining the Impact Factor (IF) of every register/bit. The IF is determined by flipping every register/bit in the pipeline register and recording the registers/bits which values are impacted by the flipping. Based on this IF data in the future design, the limited resource can be deployed on the most critical resource.

\section{2 $\quad$ Future Work}

Further development can be made in the following areas:

1. Optimize the error detection logic to reduce the clock delay and the length of the longest critical path.

2. Identify the error propagation route and determine the impact depth of each register/bit in the processor. Armed with such information, the impact of an error could be analyzed more accurately, leading to a better guide to fault tolerant design.

3. Design software to fulfill the hardware-software collaborative soft error fault tolerant scheme. Since our fault tolerant scheme only detects the soft error in the pipeline, the data errors in the memory could be covered by the software fault tolerant scheme. 


\section{Bibliogaphy and References}

[1] H. Ando, Y. Yoshida, A. Inoue, I. Sugiyama, T. Asakawa, K. Morita, T. Muta, T. Motokurumada, S. Okada, H. Yamashita, et al. A 1.3-ghz fifth-generation sparc64 microprocessor. IEEE Journal of Solid-State Circuits, 38(11):1896-1905, 2003.

[2] T. M. Austin. Diva: A reliable substrate for deep submicron microarchitecture design. In Microarchitecture, 1999. MICRO-32. Proceedings. 32nd Annual International Symposium on, pages 196-207. IEEE, 1999.

[3] K. Avery. Radiation effects point of view. In IEEE Nuclear and Space Radiation Effects Conference Short Course, Quebec City, Canada, 2009.

[4] N. D. P. Avirneni and A. K. Somani. Low overhead soft error mitigation techniques for high-performance and aggressive designs. Computers, IEEE Transactions on, 61(4):488-501, 2012.

[5] R. Baumann. Soft errors in commercial semiconductor technology: Overview and scaling trends. IEEE 2002 Reliability Physics Tutorial Notes, pages 121$121,2002$.

[6] R. Baumann. Single-event effects in advanced cmos technology. In Proc. IEEE Nuclear and Space Radiation Effects Conf. Short Course Text, pages $1-59,2005$.

[7] R. E. Bickel. Fault tolerant processing architecture, Aug. 30 2005. US Patent $6,938,183$. 
[8] D. Bossen. Cmos soft errors and server design. In IEEE 2002 Reliability Physics Tutorial Notes, Reliability Fundamentals, pages 121-121, 2002.

[9] T. Calin, M. Nicolaidis, and R. Velazco. Upset hardened memory design for submicron cmos technology. IEEE-Transactions-on-Nuclear-Science., pages 2874-8, 1996.

[10] J. Colinge. Silicon-on-insulator technology: Overview and device physics. In IEEE Nuclear Space Radiation Effects Conference, 2001.

[11] D. R. Czajkowski, P. K. Samudrala, and M. P. Pagey. Seu mitigation for reconfigurable fpgas. In 2006 IEEE Aerospace Conference, pages 7-pp. IEEE, 2006.

[12] GAISLER. Leon2 processor user's manual-xst edition. http://www.cse. wustl. edu/ roger/465M/leon2-1.0.23-xst.pdf. Accessed 2015.

[13] gaisler. Leon3 manual. http://www.cs.ucr.edu/ dalton/leon/ downloads/leon-2.3.5.pdf. Accessed April 4, 2016.

[14] V. Izosimov, I. Polian, P. Pop, P. Eles, and Z. Peng. Analysis and optimization of fault-tolerant embedded systems with hardened processors. In Design, Automation 83 Test in Europe Conference \& Exhibition, 2009. DATE'09., pages 682-687. IEEE, 2009.

[15] F. L. Kastensmidt, L. Sterpone, L. Carro, and M. S. Reorda. On the optimal design of triple modular redundancy logic for sram-based fpgas. In Proceedings of the conference on Design, Automation and Test in Europe-Volume 2, pages 1290-1295. IEEE Computer Society, 2005.

[16] S. Mitra, M. Zhang, S. Waqas, N. Seifert, B. Gill, and K. Kim. Combinational logic soft error correction. In 2006 IEEE International Test Conference, 2006.

[17] S. Mukherjee. Architecture design for soft errors. Morgan Kaufmann, 2011. 
[18] S. S. Mukherjee, M. Kontz, and S. K. Reinhardt. Detailed design and evaluation of redundant multi-threading alternatives. pages 99-110, 2002.

[19] S. S. Mukherjee, C. Weaver, J. Emer, S. K. Reinhardt, and T. Austin. A systematic methodology to compute the architectural vulnerability factors for a high-performance microprocessor. In Proceedings of the 36th annual IEEE/ACM International Symposium on Microarchitecture, page 29. IEEE Computer Society, 2003.

[20] B. Narasimham, J. W. Gambles, R. L. Shuler, B. L. Bhuva, and L. W. Massengill. Quantifying the effect of guard rings and guard drains in mitigating charge collection and charge spread. IEEE Transactions on Nuclear Science, 55(6):3456-3460, 2008.

[21] C. Poivey and J. H. Day. Radiation hardness assurance for space systems. IEEE NSREC 2002 Short Course, Jul 2002.

[22] D. K. Pradhan. Fault-tolerant computer system design. Prentice-Hall, Inc., 1996.

[23] E. Rotenberg. Ar-smt: A microarchitectural approach to fault tolerance in microprocessors. In Fault-Tolerant Computing, 1999. Digest of Papers. Twenty-Ninth Annual International Symposium on, pages 84-91. IEEE, 1999.

[24] S. Sayil. Soft Error Mechanisms, Modeling and Mitigation. Springer, 2016.

[25] J. Schwank. Total dose effects in mos devices. In IEEE NSREC Short Course, pages 1-123, 2002.

[26] X. She and K. McElvain. Time multiplexed triple modular redundancy for single event upset mitigation. IEEE Transactions on Nuclear Science, $4(56): 2443-2448,2009$.

[27] T. J. Slegel, R. M. Averill, M. A. Check, B. C. Giamei, B. W. Krumm, C. A. Krygowski, W. H. Li, J. S. Liptay, J. D. MacDougall, T. J. McPherson, et al. Ibm's s/390 g5 microprocessor design. IEEE micro, 19(2):12-23, 1999. 
[28] J. C. Smolens, B. T. Gold, B. Falsafi, and J. C. Hoe. Reunion: Complexity-effective multicore redundancy. In Proceedings of the 39th Annual IEEE/ACM International Symposium on Microarchitecture, pages 223234. IEEE Computer Society, 2006.

[29] S. M. Sze. Semiconductor devices: physics and technology. John Wiley \& Sons, 2008.

[30] Wikipedia. Coremark. https://en.wikipedia.org/wiki/Coremark. Accessed April 4, 2016.

[31] Wikipedia. Leon. https://en.wikipedia.org/wiki/LEON. Accessed May 2016.

[32] Wikipedia. Sputnik 1. https://en.wikipedia.org/wiki/Sputnik_1. Accessed April 4, 2016.

[33] Wikipedia. Tcl. https://en.wikipedia.org/wiki/Tcl. Accessed April 4, 2016.

[34] M. J. Wirthlin, A. M. Keller, C. McCloskey, P. Ridd, D. Lee, and J. Draper. Seu mitigation and validation of the leon3 soft processor using triple modular redundancy for space processing. In Proceedings of the 2016 ACM/SIGDA International Symposium on Field-Programmable Gate Arrays, pages 205214. ACM, 2016.

[35] A. Wood. Data integrity concepts, features, and technology. Tandem Division, Compaq Computer Corporation.

[36] M. Zhang, Q. Shi, and K. S. Kim. Robust system design with built-in softerror resilience. 2005 .

[37] J. F. Ziegler, H. W. Curtis, H. P. Muhlfeld, C. J. Montrose, and B. Chin. Ibm experiments in soft fails in computer electronics (1978-1994). IBM journal of research and development, 40(1):3-18, 1996. 


\section{Appendix A}

\section{Critical Resource}

Table A.1: Critical Register Statistic of All Register

\begin{tabular}{|c|c|c|c|c|c|c|c|c|c|c|c|c|c|c|c|c|c|c|}
\hline Reg Inst & $\begin{array}{l}\text { b } \\
\text { a्d }\end{array}$ & 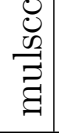 & 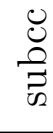 & $\underset{\nabla}{\nabla}$ & $\begin{array}{l}\stackrel{0}{0} \\
\text { त् }\end{array}$ & శ్రేี & $\tilde{0}$ & $\bar{\nabla}$ & $\overline{\dot{n}}$ & 菅 & $\overline{\widetilde{U}}$ & 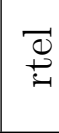 & 그 & $\vec{v}$ & $\frac{-1}{\sqrt{0}}$ & 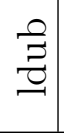 & 8 & 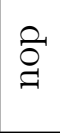 \\
\hline d.pc & $\checkmark$ & $\checkmark$ & $\checkmark$ & $\checkmark$ & $\checkmark$ & $\checkmark$ & $\checkmark$ & $\checkmark$ & $\checkmark$ & $\checkmark$ & $\checkmark$ & $\checkmark$ & $\checkmark$ & $\checkmark$ & $\checkmark$ & $\checkmark$ & $\checkmark$ & $\checkmark$ \\
\hline d.inst(0) & $\checkmark$ & $\checkmark$ & $\checkmark$ & $\checkmark$ & $\checkmark$ & $\checkmark$ & $\checkmark$ & $\checkmark$ & $\checkmark$ & $\checkmark$ & $\checkmark$ & $\checkmark$ & $\checkmark$ & $\checkmark$ & $\checkmark$ & $\checkmark$ & $\checkmark$ & $\checkmark$ \\
\hline d.cwp & $\checkmark$ & $\checkmark$ & $\checkmark$ & $\checkmark$ & $\checkmark$ & $\checkmark$ & $\checkmark$ & $\checkmark$ & $\checkmark$ & $\checkmark$ & $\checkmark$ & $\checkmark$ & $\checkmark$ & $\checkmark$ & $\checkmark$ & 2 & $\checkmark$ & $\checkmark$ \\
\hline \multicolumn{19}{|l|}{ d.awp } \\
\hline \multicolumn{19}{|l|}{ d.aw } \\
\hline \multicolumn{19}{|l|}{ d.paw } \\
\hline \multicolumn{19}{|l|}{ d.stwin } \\
\hline \multicolumn{19}{|l|}{ d.cwpmax } \\
\hline \multicolumn{19}{|l|}{ d.set } \\
\hline d.mexc & $\checkmark$ & $\checkmark$ & $\checkmark$ & $\checkmark$ & $\checkmark$ & $\checkmark$ & $\checkmark$ & $\checkmark$ & $\checkmark$ & $\checkmark$ & $\checkmark$ & $\checkmark$ & $\checkmark$ & $\checkmark$ & $\checkmark$ & $\checkmark$ & $\checkmark$ & $\checkmark$ \\
\hline d.cnt & $\checkmark$ & $\checkmark$ & $\checkmark$ & $\checkmark$ & $\checkmark$ & $\checkmark$ & $\checkmark$ & $\checkmark$ & $\checkmark$ & $\checkmark$ & $\checkmark$ & $\checkmark$ & $\checkmark$ & $\checkmark$ & $\checkmark$ & $\checkmark$ & $\checkmark$ & $\checkmark$ \\
\hline d.pv & $\checkmark$ & $\checkmark$ & $\checkmark$ & $\checkmark$ & $\checkmark$ & $\checkmark$ & $\checkmark$ & $\checkmark$ & $\checkmark$ & $\checkmark$ & $\checkmark$ & $\checkmark$ & $\checkmark$ & $\checkmark$ & $\checkmark$ & $\checkmark$ & $\checkmark$ & $\checkmark$ \\
\hline d.annul & $\checkmark$ & $\checkmark$ & $\checkmark$ & $\checkmark$ & $\checkmark$ & $\checkmark$ & $\checkmark$ & $\checkmark$ & $\checkmark$ & $\checkmark$ & $\checkmark$ & $\checkmark$ & $\checkmark$ & $\checkmark$ & $\checkmark$ & $\checkmark$ & $\checkmark$ & $\checkmark$ \\
\hline d.inull & $\checkmark$ & $\checkmark$ & $\checkmark$ & $\checkmark$ & $\checkmark$ & $\checkmark$ & $\checkmark$ & $\checkmark$ & $\checkmark$ & $r$ & $\checkmark$ & $r$ & $\checkmark$ & 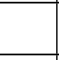 & $\checkmark$ & $r$ & $r$ & $\checkmark$ \\
\hline d.step & $\checkmark$ & $\checkmark$ & $\checkmark$ & $\checkmark$ & $\checkmark$ & $\checkmark$ & $\checkmark$ & $\checkmark$ & $\checkmark$ & $\checkmark$ & $\checkmark$ & $\checkmark$ & $\checkmark$ & $\checkmark$ & $\checkmark$ & $\checkmark$ & $\checkmark$ & $\checkmark$ \\
\hline \multicolumn{19}{|l|}{ d.divrdy } \\
\hline \multicolumn{19}{|l|}{ d.pcheld } \\
\hline d.rexen & $\checkmark$ & $\checkmark$ & $\checkmark$ & $\checkmark$ & $\checkmark$ & $\checkmark$ & $\checkmark$ & $\checkmark$ & $\checkmark$ & $\checkmark$ & $\checkmark$ & $\checkmark$ & $\checkmark$ & $\checkmark$ & $\checkmark$ & $\checkmark$ & $\checkmark$ & $\checkmark$ \\
\hline d.rexpos & $\checkmark$ & $\checkmark$ & $\checkmark$ & $\checkmark$ & $\checkmark$ & $\checkmark$ & $\checkmark$ & $\checkmark$ & $\checkmark$ & $\checkmark$ & $\checkmark$ & $\checkmark$ & $\checkmark$ & $\checkmark$ & $\checkmark$ & $\checkmark$ & $\checkmark$ & $\checkmark$ \\
\hline d.rexbuf & & & & & & & & & & & & & & & & & & \\
\hline
\end{tabular}


Appendix A. Critical Resource

Table A.1 cont'd

\begin{tabular}{|c|c|c|c|c|c|c|c|c|c|c|c|c|c|c|c|c|c|c|}
\hline Reg Inst & $\begin{array}{l}\text { bे } \\
\text { a }\end{array}$ & 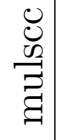 & $\begin{array}{l}\dot{U} \\
\frac{0}{3} \\
\vdots\end{array}$ & ? & 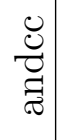 & $\begin{array}{l}\vec{\Xi} \\
\vec{\sigma}\end{array}$ & $\check{0}$ & $\bar{\sigma}$ & $\overrightarrow{\dot{m}}$ & $\begin{array}{l}\dot{0} \\
\ddot{x}\end{array}$ & $\overline{\widetilde{ల}}$ & $\begin{array}{l}\vec{D} \\
\stackrel{0}{*}=0\end{array}$ & $\Xi$ & $\vec{c}$ & $\frac{\sqrt{n}}{0}$ & $\begin{array}{l}0 \\
\vec{Z} \\
\end{array}$ & $\begin{array}{l}0 \\
0 \\
0\end{array}$ & ڤ్రి \\
\hline \multicolumn{19}{|l|}{ d.rexcnt } \\
\hline \multicolumn{19}{|c|}{ d.rexpl.opout } \\
\hline \multicolumn{19}{|c|}{ d.rexpl.szout } \\
\hline \multicolumn{19}{|c|}{ d.rexpl.ncntout } \\
\hline \multicolumn{19}{|c|}{ d.rexpl.baddr1 } \\
\hline \multicolumn{19}{|c|}{ d.rexpl.immexp } \\
\hline \multicolumn{19}{|c|}{ d.rexpl.immval } \\
\hline \multicolumn{19}{|c|}{ d.rexpl.getpc } \\
\hline \multicolumn{19}{|c|}{ d.rexpl.maskpv } \\
\hline \multicolumn{19}{|c|}{ d.rexpl.illinst } \\
\hline \multicolumn{19}{|c|}{ d.rexpl.nostep } \\
\hline \multicolumn{19}{|c|}{ d.rexpl.itovr } \\
\hline \multicolumn{19}{|c|}{ d.rexpl.leave } \\
\hline a.ctrl.pc & $\checkmark$ & $\checkmark$ & $\checkmark$ & $\checkmark$ & $\checkmark$ & $\checkmark$ & $\checkmark$ & $\checkmark$ & $\checkmark$ & $\checkmark$ & $\checkmark$ & $\checkmark$ & $\checkmark$ & $\checkmark$ & $\checkmark$ & $\checkmark$ & $\checkmark$ & $\checkmark$ \\
\hline a.ctrl.inst & $\checkmark$ & $\checkmark$ & $\checkmark$ & $\checkmark$ & $\checkmark$ & $\checkmark$ & $\checkmark$ & $\checkmark$ & $\checkmark$ & $\checkmark$ & $\checkmark$ & $\checkmark$ & $\checkmark$ & $\checkmark$ & $\checkmark$ & $\checkmark$ & $\checkmark$ & $\checkmark$ \\
\hline a.ctrl.cnt & $\checkmark$ & $\checkmark$ & $\checkmark$ & $\checkmark$ & $\checkmark$ & $\checkmark$ & $\checkmark$ & $\checkmark$ & $\checkmark$ & $\checkmark$ & $\checkmark$ & $\checkmark$ & $\checkmark$ & $\checkmark$ & $\checkmark$ & $\checkmark$ & $\checkmark$ & $\checkmark$ \\
\hline a.ctrl.rd & $\checkmark$ & $\checkmark$ & $\checkmark$ & $\checkmark$ & $\checkmark$ & $\checkmark$ & $\checkmark$ & $\checkmark$ & $\checkmark$ & $\checkmark$ & $\checkmark$ & $\checkmark$ & $\checkmark$ & $\checkmark$ & $\checkmark$ & $\checkmark$ & $\checkmark$ & $\checkmark$ \\
\hline \multicolumn{19}{|l|}{ a.ctrl.tt } \\
\hline \multicolumn{19}{|l|}{ a.ctrl.trap } \\
\hline a.ctrl.annul & $\checkmark$ & $\checkmark$ & $\checkmark$ & $\checkmark$ & $\checkmark$ & $\checkmark$ & $\checkmark$ & $\checkmark$ & $\checkmark$ & $\checkmark$ & $\checkmark$ & $\checkmark$ & $\checkmark$ & $\checkmark$ & $\checkmark$ & $\checkmark$ & $\checkmark$ & $\checkmark$ \\
\hline a.ctrl.wreg & $\checkmark$ & $\checkmark$ & $\checkmark$ & 2 & $\checkmark$ & $\checkmark$ & $\checkmark$ & $\checkmark$ & $\checkmark$ & $\checkmark$ & $\checkmark$ & $\checkmark$ & $\checkmark$ & $r$ & 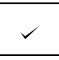 & 2 & $\checkmark$ & 2 \\
\hline a.ctrl.wicc & $\checkmark$ & $\checkmark$ & $\checkmark$ & $\checkmark$ & $\checkmark$ & $\checkmark$ & $\checkmark$ & $\checkmark$ & $\checkmark$ & $\checkmark$ & $\checkmark$ & $r$ & $\checkmark$ & $\checkmark$ & $\checkmark$ & $\checkmark$ & $\checkmark$ & $\checkmark$ \\
\hline a.ctrl.wy & $\checkmark$ & $\checkmark$ & $\checkmark$ & $\checkmark$ & $\checkmark$ & $\checkmark$ & $\checkmark$ & $\checkmark$ & $\checkmark$ & $\checkmark$ & $\checkmark$ & $\checkmark$ & $\checkmark$ & $\checkmark$ & $\checkmark$ & $\checkmark$ & $\checkmark$ & $r$ \\
\hline a.ctrl.ld & $\checkmark$ & $\checkmark$ & $\checkmark$ & $\checkmark$ & $\checkmark$ & $\checkmark$ & $\checkmark$ & $\checkmark$ & $\checkmark$ & $\checkmark$ & $\checkmark$ & $\checkmark$ & $\checkmark$ & $\checkmark$ & $\checkmark$ & $\checkmark$ & $\checkmark$ & $\checkmark$ \\
\hline a.ctrl.pv & $\checkmark$ & $\checkmark$ & $\checkmark$ & $\checkmark$ & $\checkmark$ & $\checkmark$ & $\checkmark$ & $\checkmark$ & $\checkmark$ & $\checkmark$ & $\checkmark$ & $\checkmark$ & $\checkmark$ & $\checkmark$ & $\checkmark$ & $\checkmark$ & $\checkmark$ & $\checkmark$ \\
\hline a.ctrl.rett & $\checkmark$ & $\checkmark$ & $\checkmark$ & $\checkmark$ & $\checkmark$ & $\checkmark$ & $\checkmark$ & $\checkmark$ & $\checkmark$ & $\checkmark$ & $\checkmark$ & $\checkmark$ & $\checkmark$ & $\checkmark$ & $\checkmark$ & $\checkmark$ & $\checkmark$ & $\checkmark$ \\
\hline a.ctrl.itovr & $\checkmark$ & $\checkmark$ & $\checkmark$ & $\checkmark$ & $\checkmark$ & $\checkmark$ & $\checkmark$ & $\checkmark$ & $\checkmark$ & $\checkmark$ & $\checkmark$ & $\checkmark$ & $\checkmark$ & $\checkmark$ & $\checkmark$ & $\checkmark$ & $\checkmark$ & $\checkmark$ \\
\hline \multicolumn{19}{|l|}{ a.rs1 } \\
\hline a.rfa1 & & $\checkmark$ & $\checkmark$ & & & & & & & $\checkmark$ & $\checkmark$ & & & & & & & \\
\hline a.rfa2 & & $\checkmark$ & $\checkmark$ & & & & & & & $\checkmark$ & $\checkmark$ & & & & & & & \\
\hline a.rsel1 & $\checkmark$ & $\checkmark$ & $\checkmark$ & $\checkmark$ & $\checkmark$ & $\checkmark$ & $\checkmark$ & $\checkmark$ & $\checkmark$ & $\checkmark$ & $\checkmark$ & $\checkmark$ & $\checkmark$ & $\checkmark$ & $\checkmark$ & $\checkmark$ & $\checkmark$ & $\checkmark$ \\
\hline a.rsel2 & $\checkmark$ & $\checkmark$ & $\checkmark$ & 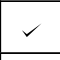 & $\checkmark$ & $\checkmark$ & $\checkmark$ & $\checkmark$ & $\checkmark$ & $\checkmark$ & $\checkmark$ & $\checkmark$ & $\checkmark$ & $\checkmark$ & $\checkmark$ & $\checkmark$ & $\checkmark$ & $\checkmark$ \\
\hline a.rfe1 & $\checkmark$ & $\checkmark$ & $\checkmark$ & $\checkmark$ & $\checkmark$ & $\checkmark$ & $\checkmark$ & $\checkmark$ & $\checkmark$ & $\checkmark$ & $\checkmark$ & $\checkmark$ & $\checkmark$ & $\checkmark$ & $\checkmark$ & $\checkmark$ & $\checkmark$ & $\checkmark$ \\
\hline a.rfe2 & $\checkmark$ & $\checkmark$ & $\checkmark$ & $\checkmark$ & $\checkmark$ & $\checkmark$ & $\checkmark$ & $\checkmark$ & $\checkmark$ & $\checkmark$ & $\checkmark$ & $\checkmark$ & $\checkmark$ & $\checkmark$ & $\checkmark$ & $\checkmark$ & $\checkmark$ & $\checkmark$ \\
\hline
\end{tabular}


Appendix A. Critical Resource

Table A.1 cont'd

\begin{tabular}{|c|c|c|c|c|c|c|c|c|c|c|c|c|c|c|c|c|c|c|}
\hline Reg Inst & $\begin{array}{l}\text { o } \\
\text { d्a }\end{array}$ & 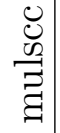 & $\begin{array}{l}\dot{U} \\
\frac{0}{7} \\
\frac{0}{3}\end{array}$ & $\vec{\tau}$ & 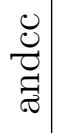 & 胥 & $\dot{0}$ & $\overline{\tilde{n}}$ & $\overrightarrow{\tilde{n}}$ & $\ddot{~}$ & $\overline{\widetilde{\mho}}$ & $\begin{array}{l}\vec{D} \\
\stackrel{D}{*}=0\end{array}$ & Z & $\dot{w}$ & $\frac{-1}{0}$ & 胥 & $\begin{array}{l}0 \\
0 \\
0\end{array}$ & 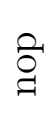 \\
\hline a.cwp & $r$ & $\checkmark$ & $\checkmark$ & $\checkmark$ & $\checkmark$ & $\checkmark$ & $\checkmark$ & $r$ & $r$ & $r$ & $\checkmark$ & $r$ & $\checkmark$ & $\checkmark$ & $\checkmark$ & $\checkmark$ & $\checkmark$ & $r$ \\
\hline \multicolumn{19}{|l|}{ a.awp } \\
\hline \multicolumn{19}{|l|}{ a.aw } \\
\hline \multicolumn{19}{|l|}{ a.paw } \\
\hline a.imm & $\checkmark$ & & & & & & & $\checkmark$ & $\checkmark$ & & & $\checkmark$ & $\checkmark$ & $\checkmark$ & $\checkmark$ & $\checkmark$ & $\checkmark$ & $\checkmark$ \\
\hline \multicolumn{19}{|l|}{ a.ldcheck1 } \\
\hline \multicolumn{19}{|l|}{ a.ldcheck2 } \\
\hline \multicolumn{19}{|l|}{ a.ldchkra } \\
\hline \multicolumn{19}{|l|}{ a.ldchkex } \\
\hline a.su & $\checkmark$ & $\checkmark$ & $\checkmark$ & $\checkmark$ & $r$ & $\checkmark$ & $\checkmark$ & $\checkmark$ & $\checkmark$ & $r$ & $\checkmark$ & $\checkmark$ & $\checkmark$ & $\checkmark$ & $\checkmark$ & $\checkmark$ & $\checkmark$ & $\checkmark$ \\
\hline a.et & $\checkmark$ & $\checkmark$ & $\checkmark$ & $\checkmark$ & $\checkmark$ & $\checkmark$ & $\checkmark$ & $\checkmark$ & $\checkmark$ & $\checkmark$ & $\checkmark$ & $r$ & $\checkmark$ & $\checkmark$ & $\checkmark$ & $\checkmark$ & $\checkmark$ & $\checkmark$ \\
\hline \multicolumn{19}{|l|}{ a.wovf } \\
\hline \multicolumn{19}{|l|}{ a.wunf } \\
\hline \multicolumn{19}{|l|}{ a.ticc } \\
\hline a.jmpl & $\checkmark$ & $\checkmark$ & $\checkmark$ & $\checkmark$ & $\checkmark$ & $\checkmark$ & $\checkmark$ & $\checkmark$ & $\checkmark$ & $\checkmark$ & $\checkmark$ & $\checkmark$ & $\checkmark$ & $\checkmark$ & $\checkmark$ & $\checkmark$ & $\checkmark$ & $\checkmark$ \\
\hline \multicolumn{19}{|l|}{ a.step } \\
\hline \multicolumn{19}{|l|}{ a.mulstart } \\
\hline \multicolumn{19}{|l|}{ a.divstart } \\
\hline a.bp & $\checkmark$ & $\checkmark$ & $\checkmark$ & $\checkmark$ & $\checkmark$ & $\checkmark$ & $\checkmark$ & $\checkmark$ & $\checkmark$ & $\checkmark$ & $\checkmark$ & $\checkmark$ & $\checkmark$ & $\checkmark$ & $\checkmark$ & $\checkmark$ & $\checkmark$ & $r$ \\
\hline \multicolumn{19}{|l|}{ a.nobp } \\
\hline a.bpimiss & $\checkmark$ & $\checkmark$ & $\checkmark$ & $\checkmark$ & $\checkmark$ & $\checkmark$ & $\checkmark$ & $\checkmark$ & $\checkmark$ & $\checkmark$ & $\checkmark$ & $\checkmark$ & $\checkmark$ & $\checkmark$ & $\checkmark$ & $\checkmark$ & $\checkmark$ & $\checkmark$ \\
\hline \multicolumn{19}{|l|}{ a.getpc } \\
\hline \multicolumn{19}{|l|}{ a.decill } \\
\hline e.ctrl.pc & $\checkmark$ & $\checkmark$ & $\checkmark$ & $\checkmark$ & $\checkmark$ & $\checkmark$ & $\checkmark$ & $\checkmark$ & $\checkmark$ & $\checkmark$ & $\checkmark$ & $\checkmark$ & $\checkmark$ & $\checkmark$ & $\checkmark$ & $\checkmark$ & $\checkmark$ & $\checkmark$ \\
\hline e.ctrl.inst & $\checkmark$ & $\checkmark$ & $\checkmark$ & $\checkmark$ & $\checkmark$ & $\checkmark$ & $\checkmark$ & $\checkmark$ & $\checkmark$ & $\checkmark$ & $\checkmark$ & $\checkmark$ & $\checkmark$ & $\checkmark$ & $\checkmark$ & $\checkmark$ & $\checkmark$ & $\checkmark$ \\
\hline e.ctrl.cnt & $\checkmark$ & $r$ & $\checkmark$ & $\checkmark$ & $\checkmark$ & $\checkmark$ & $r$ & $\checkmark$ & $r$ & $\checkmark$ & $\checkmark$ & $\checkmark$ & $\checkmark$ & $\checkmark$ & $\checkmark$ & $\checkmark$ & $\checkmark$ & $\checkmark$ \\
\hline e.ctrl.rd & $\checkmark$ & $\checkmark$ & $\checkmark$ & $\checkmark$ & $\checkmark$ & $\checkmark$ & $\checkmark$ & $\checkmark$ & $\checkmark$ & $\checkmark$ & $\checkmark$ & $\checkmark$ & $\checkmark$ & $\checkmark$ & $\checkmark$ & $\checkmark$ & $\checkmark$ & $\checkmark$ \\
\hline e.ctrl.tt & $\checkmark$ & $\checkmark$ & $\checkmark$ & $\checkmark$ & $\checkmark$ & $\checkmark$ & $\checkmark$ & $\checkmark$ & $\checkmark$ & $\checkmark$ & $\checkmark$ & $\checkmark$ & $\checkmark$ & $\checkmark$ & $\checkmark$ & $\checkmark$ & $\checkmark$ & $\checkmark$ \\
\hline e.ctrl.trap & $\checkmark$ & $\checkmark$ & $\checkmark$ & $\checkmark$ & $\checkmark$ & $\checkmark$ & $\checkmark$ & $\checkmark$ & $\checkmark$ & $\checkmark$ & $\checkmark$ & $\checkmark$ & $\checkmark$ & $\checkmark$ & $\checkmark$ & $\checkmark$ & $\checkmark$ & $\checkmark$ \\
\hline e.ctrl.annul & $\checkmark$ & $\checkmark$ & $\checkmark$ & $\checkmark$ & $\checkmark$ & $\checkmark$ & $\checkmark$ & $\checkmark$ & $\checkmark$ & $\checkmark$ & $\checkmark$ & $\checkmark$ & $\checkmark$ & $\checkmark$ & $\checkmark$ & $\checkmark$ & $\checkmark$ & $\checkmark$ \\
\hline e.ctrl.wreg & $\checkmark$ & $\checkmark$ & $\checkmark$ & $\checkmark$ & $\checkmark$ & $\checkmark$ & $\checkmark$ & $\checkmark$ & $\checkmark$ & $\checkmark$ & $\checkmark$ & $\checkmark$ & $\checkmark$ & $\checkmark$ & $\checkmark$ & $\checkmark$ & $\checkmark$ & $\checkmark$ \\
\hline e.ctrl.wicc & $r$ & $\checkmark$ & $\checkmark$ & $\checkmark$ & $\checkmark$ & $\checkmark$ & $\checkmark$ & $\checkmark$ & 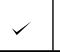 & $r$ & $\checkmark$ & $\checkmark$ & $\checkmark$ & $\checkmark$ & $\checkmark$ & $\checkmark$ & $\checkmark$ & $\checkmark$ \\
\hline e.ctrl.wy & $\checkmark$ & $\checkmark$ & $\checkmark$ & $\checkmark$ & $\checkmark$ & $\checkmark$ & $\checkmark$ & $\checkmark$ & $\checkmark$ & $\checkmark$ & $\checkmark$ & $\checkmark$ & $\checkmark$ & $\checkmark$ & $\checkmark$ & $\checkmark$ & $\checkmark$ & $\checkmark$ \\
\hline e.ctrl.ld & $\checkmark$ & $\checkmark$ & $\checkmark$ & $\checkmark$ & $\checkmark$ & $\checkmark$ & $\checkmark$ & $\checkmark$ & $\checkmark$ & $\checkmark$ & $\checkmark$ & $\checkmark$ & $\checkmark$ & $\checkmark$ & $\checkmark$ & $\checkmark$ & $\checkmark$ & $\checkmark$ \\
\hline
\end{tabular}


Appendix A. Critical Resource

Table A.1 cont'd

\begin{tabular}{|c|c|c|c|c|c|c|c|c|c|c|c|c|c|c|c|c|c|c|}
\hline Reg Inst & $\begin{array}{l}\text { a } \\
\text { g }\end{array}$ & 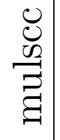 & $\begin{array}{l}\dot{U} \\
\frac{0}{Z} \\
\vec{\omega}\end{array}$ & $\frac{7}{\pi}$ & 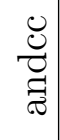 & జేశ్ & $\ddot{0}$ & $\bar{\sigma}$ & $\overline{\dot{\omega}}$ & 容 & $\underset{\widetilde{U}}{\overline{\mathcal{U}}}$ & $\begin{array}{l}\vec{D} \\
\stackrel{ \pm}{*}\end{array}$ & $\Xi$ & $\overrightarrow{0}$ & $\frac{\theta}{0}$ & $\frac{O}{\Xi}$ & $\stackrel{0}{0}$ & : \\
\hline e.ctrl.pv & $\checkmark$ & $\checkmark$ & $\checkmark$ & $\checkmark$ & $\checkmark$ & $\checkmark$ & $\checkmark$ & $\checkmark$ & $\checkmark$ & $\checkmark$ & $\checkmark$ & $\checkmark$ & $\checkmark$ & $\checkmark$ & $\checkmark$ & $\checkmark$ & $\checkmark$ & $\checkmark$ \\
\hline e.ctrl.rett & $\checkmark$ & $\checkmark$ & $\checkmark$ & $\checkmark$ & $\checkmark$ & $\checkmark$ & $\checkmark$ & $\checkmark$ & $\checkmark$ & $\checkmark$ & $\checkmark$ & $\checkmark$ & $r$ & $\checkmark$ & $\checkmark$ & $\checkmark$ & $\checkmark$ & $\checkmark$ \\
\hline e.ctrl.itovr & $\checkmark$ & $\checkmark$ & $\checkmark$ & $\checkmark$ & $\checkmark$ & $\checkmark$ & $\checkmark$ & $r$ & $\checkmark$ & $\checkmark$ & $r$ & $r$ & $\checkmark$ & $\checkmark$ & $r$ & $\checkmark$ & $\checkmark$ & $\checkmark$ \\
\hline e.op1 & $\checkmark$ & $\checkmark$ & $\checkmark$ & $\checkmark$ & $\checkmark$ & $\checkmark$ & $\checkmark$ & $\checkmark$ & $\checkmark$ & $\checkmark$ & $\checkmark$ & $\checkmark$ & $\checkmark$ & $\checkmark$ & $\checkmark$ & $\checkmark$ & $\checkmark$ & $\checkmark$ \\
\hline e.op2 & $\checkmark$ & & $\checkmark$ & $\checkmark$ & $\checkmark$ & $\checkmark$ & $\checkmark$ & $\checkmark$ & $\checkmark$ & $\checkmark$ & $\checkmark$ & $\checkmark$ & $\checkmark$ & $\checkmark$ & $\checkmark$ & $\checkmark$ & $\checkmark$ & $\checkmark$ \\
\hline e.aluop & $\checkmark$ & & & & & & $\checkmark$ & & $\checkmark$ & $\checkmark$ & $\checkmark$ & & & & & & & $\checkmark$ \\
\hline e.alusel & $\checkmark$ & . & $\checkmark$ & $\checkmark$ & $\checkmark$ & $\checkmark$ & $\checkmark$ & $\checkmark$ & $\checkmark$ & $\checkmark$ & $\checkmark$ & & $\checkmark$ & $\checkmark$ & $\checkmark$ & $\checkmark$ & $\checkmark$ & $\checkmark$ \\
\hline e.aluadd & & $\checkmark$ & $\checkmark$ & & & & & & & & & & & & & & & \\
\hline e.alucin & $\checkmark$ & $\checkmark$ & $\checkmark$ & $\checkmark$ & $\checkmark$ & $\checkmark$ & $\checkmark$ & $\checkmark$ & $\checkmark$ & $\checkmark$ & $\checkmark$ & $\checkmark$ & $\checkmark$ & $\checkmark$ & $\checkmark$ & $\checkmark$ & $\checkmark$ & $\checkmark$ \\
\hline e.ldbp1 & $\checkmark$ & $\checkmark$ & $\checkmark$ & $\checkmark$ & $\checkmark$ & $\checkmark$ & $\checkmark$ & $\checkmark$ & $\checkmark$ & $\checkmark$ & $\checkmark$ & $\checkmark$ & $\checkmark$ & $\checkmark$ & $\checkmark$ & $\checkmark$ & $\checkmark$ & $\checkmark$ \\
\hline e.ldbp2 & $\checkmark$ & $\checkmark$ & $\checkmark$ & $\checkmark$ & $\checkmark$ & $\checkmark$ & $\checkmark$ & $\checkmark$ & $r$ & $\checkmark$ & $\checkmark$ & $\checkmark$ & $\checkmark$ & $\checkmark$ & $\checkmark$ & $\checkmark$ & $\checkmark$ & $\checkmark$ \\
\hline \multicolumn{19}{|l|}{ e.invop2 } \\
\hline e.shcnt & & & & & & & & $\checkmark$ & $\checkmark$ & & & & & & & & & \\
\hline \multicolumn{19}{|l|}{ e.sari } \\
\hline e.shleft & & & & & & & & $\checkmark$ & & & & & & & & & & \\
\hline e.ymsb & & $\checkmark$ & & & & & & & & & & & & & & & & \\
\hline \multicolumn{19}{|l|}{ e.rd } \\
\hline e.jmpl & $\checkmark$ & $\checkmark$ & & $\checkmark$ & & & & & & & & $\checkmark$ & $\checkmark$ & $\checkmark$ & $\checkmark$ & $\checkmark$ & $\checkmark$ & $\checkmark$ \\
\hline e.su & $\checkmark$ & $\checkmark$ & $\checkmark$ & $\checkmark$ & $\checkmark$ & $\checkmark$ & $\checkmark$ & $\checkmark$ & $\checkmark$ & $\checkmark$ & $\checkmark$ & $\checkmark$ & $\checkmark$ & $\checkmark$ & $\checkmark$ & $\checkmark$ & $\checkmark$ & $\checkmark$ \\
\hline \multicolumn{19}{|l|}{ e.et } \\
\hline \multicolumn{19}{|l|}{ e.cwp } \\
\hline \multicolumn{19}{|l|}{ e.awp } \\
\hline \multicolumn{19}{|l|}{ e.aw } \\
\hline \multicolumn{19}{|l|}{ e.paw } \\
\hline \multicolumn{19}{|l|}{ e.icc } \\
\hline e.mulstep & & $\checkmark$ & & & & & & & & & & & & & & & & \\
\hline \multicolumn{19}{|l|}{ e.mul } \\
\hline e.mac & $r$ & $\checkmark$ & $\checkmark$ & $\checkmark$ & $r$ & $r$ & $\checkmark$ & $r$ & $\checkmark$ & $\checkmark$ & $\checkmark$ & $\checkmark$ & $\checkmark$ & $r$ & $\checkmark$ & $\checkmark$ & $\checkmark$ & $\checkmark$ \\
\hline e.bp & $\checkmark$ & $\checkmark$ & $\checkmark$ & $\checkmark$ & $\checkmark$ & $\checkmark$ & $\checkmark$ & $r$ & $\checkmark$ & $\checkmark$ & $\checkmark$ & $\checkmark$ & $\checkmark$ & $\checkmark$ & $r$ & $\checkmark$ & $\checkmark$ & $\checkmark$ \\
\hline \multicolumn{19}{|l|}{ e.rfe1 } \\
\hline \multicolumn{19}{|l|}{ e.rfe2 } \\
\hline m.ctrl.pc & $\checkmark$ & $\checkmark$ & $\checkmark$ & $\checkmark$ & $\checkmark$ & $\checkmark$ & $\checkmark$ & $\checkmark$ & $\checkmark$ & $\checkmark$ & $\checkmark$ & $\checkmark$ & $\checkmark$ & $\checkmark$ & $\checkmark$ & $\checkmark$ & $\checkmark$ & $\checkmark$ \\
\hline m.ctrl.inst & $\checkmark$ & $\checkmark$ & $\checkmark$ & $\checkmark$ & $\checkmark$ & $\checkmark$ & $\checkmark$ & $\checkmark$ & $\checkmark$ & $\checkmark$ & $\checkmark$ & $\checkmark$ & $\checkmark$ & $\checkmark$ & $\checkmark$ & $\checkmark$ & $\checkmark$ & $\checkmark$ \\
\hline m.ctrl.cnt & $\checkmark$ & $\checkmark$ & $\checkmark$ & $\checkmark$ & $\checkmark$ & $\checkmark$ & $r$ & $\checkmark$ & $\checkmark$ & $\checkmark$ & $\checkmark$ & $\checkmark$ & $\checkmark$ & $\checkmark$ & $\checkmark$ & $\checkmark$ & $\checkmark$ & $\checkmark$ \\
\hline
\end{tabular}


Appendix A. Critical Resource

Table A.1 cont'd

\begin{tabular}{|c|c|c|c|c|c|c|c|c|c|c|c|c|c|c|c|c|c|c|}
\hline Reg Inst & 总 & 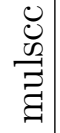 & $\begin{array}{l}\dot{U} \\
\frac{0}{7} \\
\frac{0}{3}\end{array}$ & $\vec{\nabla}$ & 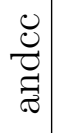 & 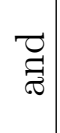 & $\dot{0}$ & $\bar{\nabla}$ & $\overrightarrow{\dot{\omega}}$ & $\ddot{~}$ & $\overline{\widetilde{U}}$ & 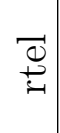 & $\underline{Z}$ & $\overrightarrow{w_{n}}$ & $\frac{-1}{0}$ & $\frac{0}{3}$ & $\begin{array}{l}0 \\
0 \\
0\end{array}$ & 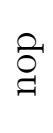 \\
\hline m.ctrl.rd & $r$ & $\checkmark$ & $r$ & $\checkmark$ & $r$ & $r$ & $r$ & $r$ & $r$ & $r$ & $\checkmark$ & $r$ & $\checkmark$ & $\checkmark$ & $\checkmark$ & $\checkmark$ & $r$ & $r$ \\
\hline m.ctrl.tt & $\checkmark$ & $\checkmark$ & $\checkmark$ & $\checkmark$ & $\checkmark$ & $\checkmark$ & $\checkmark$ & $\checkmark$ & $\checkmark$ & $\checkmark$ & $\checkmark$ & $\checkmark$ & $\checkmark$ & $\checkmark$ & $\checkmark$ & $\checkmark$ & $\checkmark$ & $\checkmark$ \\
\hline m.ctrl.trap & $\checkmark$ & $\checkmark$ & $\checkmark$ & $\checkmark$ & $\checkmark$ & $\checkmark$ & $\checkmark$ & $\checkmark$ & 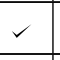 & $\checkmark$ & $\checkmark$ & $\checkmark$ & $\checkmark$ & $\checkmark$ & $\checkmark$ & $\checkmark$ & $\checkmark$ & $\checkmark$ \\
\hline m.ctrl.annul & $\checkmark$ & $\checkmark$ & $\checkmark$ & $\checkmark$ & $\checkmark$ & $\checkmark$ & $\checkmark$ & $\checkmark$ & $\checkmark$ & $\checkmark$ & $\checkmark$ & $\checkmark$ & $\checkmark$ & $\checkmark$ & $\checkmark$ & $r$ & $\checkmark$ & $\checkmark$ \\
\hline m.ctrl.wreg & $\checkmark$ & $\checkmark$ & $\checkmark$ & $\checkmark$ & $\checkmark$ & $\checkmark$ & $\checkmark$ & $\checkmark$ & $\checkmark$ & $r$ & $\checkmark$ & $\checkmark$ & $\checkmark$ & 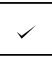 & $\checkmark$ & $\checkmark$ & $\checkmark$ & $\checkmark$ \\
\hline m.ctrl.wicc & $\checkmark$ & $\checkmark$ & $\checkmark$ & $\checkmark$ & $\checkmark$ & $\checkmark$ & $\checkmark$ & $\checkmark$ & $\checkmark$ & $\checkmark$ & $\checkmark$ & $\checkmark$ & $\checkmark$ & $\checkmark$ & $\checkmark$ & $\checkmark$ & $\checkmark$ & $\checkmark$ \\
\hline m.ctrl.wy & $\checkmark$ & $\checkmark$ & $\checkmark$ & $\checkmark$ & $\checkmark$ & $\checkmark$ & $\checkmark$ & $\checkmark$ & $\checkmark$ & $\checkmark$ & $\checkmark$ & $\checkmark$ & $\checkmark$ & $\checkmark$ & $\checkmark$ & $\checkmark$ & $\checkmark$ & $\checkmark$ \\
\hline m.ctrl.ld & $\checkmark$ & $\checkmark$ & $\checkmark$ & $\checkmark$ & $\checkmark$ & $\checkmark$ & $\checkmark$ & $\checkmark$ & $\checkmark$ & $\checkmark$ & $\checkmark$ & $\checkmark$ & $\checkmark$ & $\checkmark$ & $\checkmark$ & $\checkmark$ & $\checkmark$ & $\checkmark$ \\
\hline m.ctrl.pv & $\checkmark$ & $\checkmark$ & $\checkmark$ & $\checkmark$ & $\checkmark$ & $\checkmark$ & $\checkmark$ & $\checkmark$ & $\checkmark$ & $\checkmark$ & $\checkmark$ & $\checkmark$ & $\checkmark$ & $\checkmark$ & $\checkmark$ & $\checkmark$ & $\checkmark$ & $\checkmark$ \\
\hline m.ctrl.rett & $\checkmark$ & $\checkmark$ & $\checkmark$ & $\checkmark$ & $\checkmark$ & $\checkmark$ & $\checkmark$ & $\checkmark$ & $\checkmark$ & $\checkmark$ & $\checkmark$ & $\checkmark$ & $\checkmark$ & $\checkmark$ & $\checkmark$ & $\checkmark$ & $\checkmark$ & $\checkmark$ \\
\hline m.ctrl.itovr & $\checkmark$ & $\checkmark$ & $\checkmark$ & $\checkmark$ & $\checkmark$ & $\checkmark$ & $\checkmark$ & $\checkmark$ & $\checkmark$ & $\checkmark$ & $\checkmark$ & $\checkmark$ & $\checkmark$ & $\checkmark$ & $\checkmark$ & $\checkmark$ & $\checkmark$ & $\checkmark$ \\
\hline m.result & $\checkmark$ & $\checkmark$ & $\checkmark$ & $\checkmark$ & $\checkmark$ & $\checkmark$ & $\checkmark$ & $\checkmark$ & $\checkmark$ & $\checkmark$ & $\checkmark$ & $\checkmark$ & $\checkmark$ & $\checkmark$ & $\checkmark$ & $\checkmark$ & $\checkmark$ & $\checkmark$ \\
\hline $\mathrm{m} . \mathrm{y}$ & $\checkmark$ & $\checkmark$ & $\checkmark$ & $\checkmark$ & $\checkmark$ & $\checkmark$ & $\checkmark$ & $\checkmark$ & $\checkmark$ & $\checkmark$ & $\checkmark$ & $\checkmark$ & $\checkmark$ & $\checkmark$ & $\checkmark$ & $\checkmark$ & $\checkmark$ & $\checkmark$ \\
\hline m.icc & $\checkmark$ & $\checkmark$ & $\checkmark$ & $\checkmark$ & $\checkmark$ & $\checkmark$ & $\checkmark$ & $\checkmark$ & $\checkmark$ & $\checkmark$ & $\checkmark$ & $\checkmark$ & $\checkmark$ & $\checkmark$ & $\checkmark$ & $\checkmark$ & $\checkmark$ & $\checkmark$ \\
\hline m.nalign & & & & & & & & & & & & $\checkmark$ & & & & & & \\
\hline m.dci.signed & $\checkmark$ & $\checkmark$ & $\checkmark$ & $\checkmark$ & $\checkmark$ & $\checkmark$ & $\checkmark$ & $\checkmark$ & $\checkmark$ & $\checkmark$ & $\checkmark$ & $\checkmark$ & $\checkmark$ & $\checkmark$ & $\checkmark$ & $\checkmark$ & $\checkmark$ & $\checkmark$ \\
\hline m.dci.enaddr & $\checkmark$ & $\checkmark$ & $\checkmark$ & 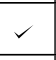 & $\checkmark$ & $\checkmark$ & $\checkmark$ & $\checkmark$ & $\checkmark$ & $\checkmark$ & $\checkmark$ & $\checkmark$ & $\checkmark$ & $\checkmark$ & $r$ & $\checkmark$ & $\checkmark$ & $\checkmark$ \\
\hline m.dci.read & $\checkmark$ & $\checkmark$ & $\checkmark$ & $\checkmark$ & $\checkmark$ & $\checkmark$ & $\checkmark$ & $\checkmark$ & $\checkmark$ & $\checkmark$ & $\checkmark$ & $\checkmark$ & $\checkmark$ & $\checkmark$ & $\checkmark$ & $\checkmark$ & $\checkmark$ & $\checkmark$ \\
\hline m.dci.write & $\checkmark$ & $r$ & $r$ & $r$ & $r$ & $r$ & $r$ & $r$ & $r$ & $r$ & $r$ & $r$ & $r$ & $\checkmark$ & $\checkmark$ & 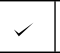 & $\checkmark$ & $\checkmark$ \\
\hline m.dci.lock & $\checkmark$ & $\checkmark$ & $\checkmark$ & $\checkmark$ & $\checkmark$ & $\checkmark$ & $\checkmark$ & $\checkmark$ & $\checkmark$ & $\checkmark$ & $\checkmark$ & $\checkmark$ & $\checkmark$ & $\checkmark$ & $\checkmark$ & $\checkmark$ & $\checkmark$ & $\checkmark$ \\
\hline m.dci.dsuen & $\checkmark$ & $\checkmark$ & $\checkmark$ & $\checkmark$ & $\checkmark$ & $\checkmark$ & $\checkmark$ & $\checkmark$ & $\checkmark$ & $\checkmark$ & $\checkmark$ & $\checkmark$ & $\checkmark$ & $\checkmark$ & $\checkmark$ & $\checkmark$ & $r$ & $\checkmark$ \\
\hline m.dci.size & $\checkmark$ & $r$ & $\checkmark$ & $\checkmark$ & $\checkmark$ & $\checkmark$ & $\checkmark$ & $\checkmark$ & $\checkmark$ & $\checkmark$ & $\checkmark$ & $\checkmark$ & $\checkmark$ & $\checkmark$ & $\checkmark$ & $\checkmark$ & $\checkmark$ & $\checkmark$ \\
\hline m.dci.asi & $\checkmark$ & $\checkmark$ & $\checkmark$ & $\checkmark$ & $\checkmark$ & $\checkmark$ & $\checkmark$ & $\checkmark$ & $\checkmark$ & $\checkmark$ & $\checkmark$ & $\checkmark$ & $\checkmark$ & $\checkmark$ & $\checkmark$ & $\checkmark$ & $\checkmark$ & $\checkmark$ \\
\hline m.werr & $\checkmark$ & $r$ & $\checkmark$ & & & $\checkmark$ & $\checkmark$ & $\checkmark$ & $\checkmark$ & 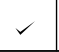 & 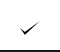 & $\checkmark$ & $\checkmark$ & $\checkmark$ & $\checkmark$ & & & \\
\hline m.wcwp & $\checkmark$ & & & $\checkmark$ & $\checkmark$ & $\checkmark$ & $\checkmark$ & $\checkmark$ & $\checkmark$ & $\checkmark$ & $\checkmark$ & $\checkmark$ & $\checkmark$ & $\checkmark$ & $\checkmark$ & $\checkmark$ & $\checkmark$ & $\checkmark$ \\
\hline \multicolumn{19}{|l|}{ m.wawp } \\
\hline \multicolumn{19}{|l|}{ m.irqen } \\
\hline \multicolumn{19}{|l|}{ m.irqen2 } \\
\hline m.mac & $\checkmark$ & $\checkmark$ & $\checkmark$ & $\checkmark$ & $\checkmark$ & $\checkmark$ & $\checkmark$ & $\checkmark$ & $\checkmark$ & $\checkmark$ & $\checkmark$ & 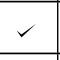 & $\checkmark$ & $\checkmark$ & $\checkmark$ & $\checkmark$ & $\checkmark$ & 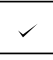 \\
\hline \multicolumn{19}{|l|}{ m.divz } \\
\hline m.su & $\checkmark$ & $\checkmark$ & $\checkmark$ & $\checkmark$ & $\checkmark$ & 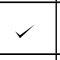 & $r$ & $\checkmark$ & 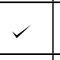 & $\checkmark$ & $\checkmark$ & $\checkmark$ & $\checkmark$ & $\checkmark$ & $\checkmark$ & $\checkmark$ & $\checkmark$ & $\checkmark$ \\
\hline \multicolumn{19}{|l|}{ m.mul } \\
\hline m.casa & $\checkmark$ & $\checkmark$ & $\checkmark$ & $\checkmark$ & $\checkmark$ & $\checkmark$ & $\checkmark$ & $\checkmark$ & $\checkmark$ & $\checkmark$ & $\checkmark$ & $\checkmark$ & $\checkmark$ & $\checkmark$ & & $\checkmark$ & $\checkmark$ & \\
\hline m.casaz & & & & & & & & & & & & & & & & & & \\
\hline
\end{tabular}


Appendix A. Critical Resource

Table A.1 cont'd

\begin{tabular}{|c|c|c|c|c|c|c|c|c|c|c|c|c|c|c|c|c|c|c|}
\hline Reg Inst & $\begin{array}{l}\vec{a} \\
\text { a }\end{array}$ & 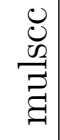 & 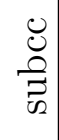 & శ & 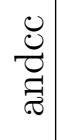 & $\overrightarrow{\widetilde{z}}$ & ठี & $\bar{\omega}$ & $\overline{\tilde{\omega}}$ & $\begin{array}{l}\dot{0} \\
\ddot{x}\end{array}$ & $\overline{\widetilde{ల}}$ & $\begin{array}{l}\vec{D} \\
\stackrel{D}{*}=1\end{array}$ & $\Xi$ & $\overrightarrow{0}$ & $\frac{\sqrt{n}}{\sqrt{0}}$ & $\frac{O}{\Xi}$ & 8 & : \\
\hline \multicolumn{19}{|l|}{ m.rexnalign } \\
\hline x.ctrl.pc & 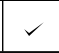 & $r$ & $r$ & $r$ & $r$ & $r$ & $r$ & $r$ & $r$ & $r$ & $r$ & $r$ & $\checkmark$ & $r$ & $r$ & $\checkmark$ & $\checkmark$ & 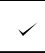 \\
\hline \multicolumn{19}{|l|}{ x.ctrl.inst } \\
\hline \multicolumn{19}{|l|}{ x.ctrl.cnt } \\
\hline x.ctrl.rd & $\checkmark$ & $r$ & $\checkmark$ & $\checkmark$ & $\checkmark$ & $\checkmark$ & $\checkmark$ & $\checkmark$ & $\checkmark$ & $r$ & 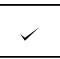 & $r$ & $r$ & $r$ & $r$ & $\checkmark$ & $r$ & $\checkmark$ \\
\hline \multicolumn{19}{|l|}{ x.ctrl.tt } \\
\hline x.ctrl.trap & $r$ & $\checkmark$ & 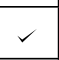 & $\checkmark$ & $\checkmark$ & $\checkmark$ & 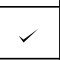 & $\checkmark$ & $\checkmark$ & $\checkmark$ & 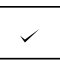 & 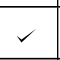 & $\checkmark$ & $\checkmark$ & $\checkmark$ & $\checkmark$ & $\checkmark$ & 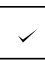 \\
\hline x.ctrl.annul & $\checkmark$ & $\checkmark$ & $\checkmark$ & $\checkmark$ & & & $\checkmark$ & $\checkmark$ & $\checkmark$ & $\checkmark$ & $\checkmark$ & & $\checkmark$ & & $\checkmark$ & $\checkmark$ & $\checkmark$ & \\
\hline x.ctrl.wreg & $\checkmark$ & $\checkmark$ & $\checkmark$ & $\checkmark$ & & & $\checkmark$ & $\checkmark$ & $\checkmark$ & $\checkmark$ & $\checkmark$ & & & $r$ & & & & \\
\hline x.ctrl.wicc & & $\checkmark$ & & & & & & & & & & & & & & & & \\
\hline x.ctrl.wy & & $\checkmark$ & & & & & & & & & & & & & & & & \\
\hline x.ctrl.ld & $\checkmark$ & $\checkmark$ & $\checkmark$ & $\checkmark$ & $\checkmark$ & $\checkmark$ & $r$ & $\checkmark$ & $\checkmark$ & $r$ & 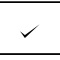 & $r$ & $r$ & 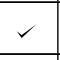 & $\checkmark$ & $\checkmark$ & $\checkmark$ & $\checkmark$ \\
\hline \multicolumn{19}{|l|}{ x.ctrl.pv } \\
\hline \multicolumn{19}{|l|}{ x.ctrl.rett } \\
\hline \multicolumn{19}{|l|}{ x.ctrl.itovr } \\
\hline x.result & $\checkmark$ & 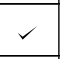 & $\checkmark$ & $\checkmark$ & $\checkmark$ & $\checkmark$ & $r$ & $\checkmark$ & $\checkmark$ & $\checkmark$ & $\checkmark$ & $\checkmark$ & & $\checkmark$ & & & & $r$ \\
\hline x.y & & $\checkmark$ & & & & & & & & & & & & & & & & \\
\hline x.icc & & & 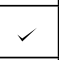 & & $\checkmark$ & & & & & & & & & & & & & \\
\hline \multicolumn{19}{|l|}{ x.annulall } \\
\hline x.data $(0)$ & & & & & & & & & & & & & $r$ & & $r$ & $r$ & $\checkmark$ & \\
\hline \multicolumn{19}{|l|}{ x.set } \\
\hline x.mexc & $\checkmark$ & $\checkmark$ & $r$ & $\checkmark$ & $\checkmark$ & $\checkmark$ & $r$ & $r$ & $\checkmark$ & $\checkmark$ & $\checkmark$ & $r$ & $\checkmark$ & $r$ & $r$ & $\checkmark$ & $\checkmark$ & $r$ \\
\hline \multicolumn{19}{|l|}{ x.dci.signed } \\
\hline \multicolumn{19}{|l|}{ x.dci.enaddr } \\
\hline \multicolumn{19}{|l|}{ x.dci.read } \\
\hline \multicolumn{19}{|l|}{ x.dci.write } \\
\hline \multicolumn{19}{|l|}{ x.dci.lock } \\
\hline \multicolumn{19}{|l|}{ x.dci.dsuen } \\
\hline \multicolumn{19}{|l|}{ x.dci.size } \\
\hline \multicolumn{19}{|l|}{ x.dci.asi } \\
\hline \multicolumn{19}{|l|}{ x.laddr } \\
\hline \multicolumn{19}{|l|}{ x.rstate } \\
\hline \multicolumn{19}{|l|}{ x.npc } \\
\hline x.intack & $\checkmark$ & $\checkmark$ & $\checkmark$ & $\checkmark$ & & & $r$ & $\checkmark$ & $\checkmark$ & $r$ & $\checkmark$ & & & $\checkmark$ & & & & \\
\hline
\end{tabular}


Appendix A. Critical Resource

Table A.1 cont'd

\begin{tabular}{|c|c|c|c|c|c|c|c|c|c|c|c|c|c|c|c|c|c|c|}
\hline Reg Inst & 总 & 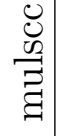 & $\begin{array}{l}\dot{U} \\
\frac{0}{\vec{\sigma}} \\
\tilde{\omega}\end{array}$ & 疋 & 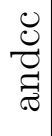 & $\begin{array}{l}\vec{Z} \\
\vec{\tau}\end{array}$ & $\check{0}$ & $\overline{\tilde{n}}$ & $\overline{\dot{\tilde{n}}}$ & $\stackrel{\overrightarrow{0}}{\not}$ & $\overline{\widetilde{U}}$ & $\underset{\sim}{\stackrel{D}{ \pm}}$ & 드 & $\overrightarrow{U S}$ & $\begin{array}{l}\frac{-1}{d} \\
\frac{d}{2}\end{array}$ & $\stackrel{0}{\underline{Z}}$ & : & ڤ్ \\
\hline \multicolumn{19}{|l|}{ x.ipend } \\
\hline \multicolumn{19}{|l|}{ x.mac } \\
\hline \multicolumn{19}{|l|}{ x.debug } \\
\hline x.nerror & $\checkmark$ & $\checkmark$ & $\checkmark$ & $\checkmark$ & $\checkmark$ & $\checkmark$ & $\checkmark$ & $\checkmark$ & $\checkmark$ & $\checkmark$ & $\checkmark$ & $\checkmark$ & $\checkmark$ & $\checkmark$ & $\checkmark$ & $\checkmark$ & $\checkmark$ & $\checkmark$ \\
\hline \multicolumn{19}{|l|}{ w.s.cwp } \\
\hline w.s.icc & $\checkmark$ & & & $\checkmark$ & & $\checkmark$ & $\checkmark$ & $\checkmark$ & $\checkmark$ & $\checkmark$ & $\checkmark$ & $\checkmark$ & $\checkmark$ & $\checkmark$ & $\checkmark$ & $\checkmark$ & $\checkmark$ & $\checkmark$ \\
\hline w.s.tt & $\checkmark$ & $\checkmark$ & $\checkmark$ & $\checkmark$ & $\checkmark$ & $\checkmark$ & $\checkmark$ & $\checkmark$ & $\checkmark$ & $\checkmark$ & $\checkmark$ & $\checkmark$ & $\checkmark$ & $\checkmark$ & $\checkmark$ & $\checkmark$ & $\checkmark$ & $\checkmark$ \\
\hline \multicolumn{19}{|l|}{ w.s.tba } \\
\hline w.s.wim & & & & $\checkmark$ & & $\checkmark$ & & & $\checkmark$ & & $\checkmark$ & & & & & & & \\
\hline w.s.pil & $\checkmark$ & $\checkmark$ & . & $\checkmark$ & $\checkmark$ & $\checkmark$ & $\checkmark$ & & $\checkmark$ & $\checkmark$ & $\checkmark$ & $\checkmark$ & & $\checkmark$ & & & & $\checkmark$ \\
\hline W.s.ec & $\checkmark$ & $\checkmark$ & $\checkmark$ & $\checkmark$ & $\checkmark$ & $\checkmark$ & $\checkmark$ & $\checkmark$ & $\checkmark$ & $\checkmark$ & $\checkmark$ & $\checkmark$ & & $\checkmark$ & $\checkmark$ & & & $\checkmark$ \\
\hline w.s.ef & $\checkmark$ & $\checkmark$ & $\checkmark$ & $\checkmark$ & $\checkmark$ & $\checkmark$ & $\checkmark$ & $\checkmark$ & $\checkmark$ & $\checkmark$ & $\checkmark$ & $\checkmark$ & $\checkmark$ & $\checkmark$ & $\checkmark$ & $\checkmark$ & $\checkmark$ & $\checkmark$ \\
\hline w.s.ps & $\checkmark$ & $\checkmark$ & $\checkmark$ & $\checkmark$ & $\checkmark$ & $\checkmark$ & $\checkmark$ & $\checkmark$ & $\checkmark$ & $\checkmark$ & $\checkmark$ & $\checkmark$ & & $\checkmark$ & $\checkmark$ & & & $\checkmark$ \\
\hline W.s.s & $\checkmark$ & $\checkmark$ & $\checkmark$ & $\checkmark$ & $\checkmark$ & $\checkmark$ & $\checkmark$ & $\checkmark$ & $\checkmark$ & $\checkmark$ & $\checkmark$ & $\checkmark$ & $\checkmark$ & $\checkmark$ & $\checkmark$ & $\checkmark$ & $\checkmark$ & $\checkmark$ \\
\hline w.s.et & $\checkmark$ & $\checkmark$ & $\checkmark$ & $\checkmark$ & $\checkmark$ & $\checkmark$ & $\checkmark$ & $\checkmark$ & $\checkmark$ & $\checkmark$ & $\checkmark$ & $\checkmark$ & $\checkmark$ & $\checkmark$ & $\checkmark$ & $\checkmark$ & $\checkmark$ & $\checkmark$ \\
\hline w.s.y & $\checkmark$ & $\checkmark$ & $\checkmark$ & $\checkmark$ & $\checkmark$ & $\checkmark$ & $\checkmark$ & $\checkmark$ & $\checkmark$ & $\checkmark$ & $\checkmark$ & $\checkmark$ & $\checkmark$ & $\checkmark$ & $\checkmark$ & $\checkmark$ & $\checkmark$ & $\checkmark$ \\
\hline w.s.asr18 & $\checkmark$ & $\checkmark$ & $\checkmark$ & $\checkmark$ & $\checkmark$ & $\checkmark$ & $\checkmark$ & $\checkmark$ & $\checkmark$ & $\checkmark$ & $\checkmark$ & $\checkmark$ & & $\checkmark$ & $\checkmark$ & & & $\checkmark$ \\
\hline w.s.svt & $\checkmark$ & $\checkmark$ & $\checkmark$ & $\checkmark$ & $\checkmark$ & $\checkmark$ & $\checkmark$ & $\checkmark$ & $\checkmark$ & $\checkmark$ & $\checkmark$ & $\checkmark$ & & $\checkmark$ & $\checkmark$ & & & $\checkmark$ \\
\hline w.s.dwt & $\checkmark$ & $\checkmark$ & $\checkmark$ & $\checkmark$ & $\checkmark$ & $\checkmark$ & $\checkmark$ & $\checkmark$ & $\checkmark$ & $\checkmark$ & $\checkmark$ & $\checkmark$ & & $\checkmark$ & $\checkmark$ & & & $\checkmark$ \\
\hline w.s.dbp & $\checkmark$ & $\checkmark$ & $\checkmark$ & $\checkmark$ & $\checkmark$ & $\checkmark$ & $\checkmark$ & $\checkmark$ & $\checkmark$ & 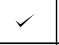 & $\checkmark$ & $\checkmark$ & & $\checkmark$ & $\checkmark$ & & & 2 \\
\hline w.s.dbprepl & $\checkmark$ & $\checkmark$ & $\checkmark$ & $\checkmark$ & $\checkmark$ & $\checkmark$ & $\checkmark$ & $\checkmark$ & $\checkmark$ & $\checkmark$ & $\checkmark$ & $\checkmark$ & & $\checkmark$ & $\checkmark$ & & & $\checkmark$ \\
\hline \multicolumn{19}{|l|}{ w.s.rexdis } \\
\hline \multicolumn{19}{|l|}{ w.s.rextrap } \\
\hline \multicolumn{19}{|l|}{ w.s.aw } \\
\hline \multicolumn{19}{|l|}{ w.s.paw } \\
\hline \multicolumn{19}{|l|}{ w.s.awp } \\
\hline w.s.stwin & & & & & & $\checkmark$ & & & & & $\checkmark$ & & & & & & & \\
\hline w.s.cwpmax & $\checkmark$ & $\checkmark$ & & $\checkmark$ & & $\checkmark$ & $\checkmark$ & & $\checkmark$ & & $\checkmark$ & $\checkmark$ & & $\checkmark$ & & & & $\checkmark$ \\
\hline w.result & $r$ & $\checkmark$ & $\checkmark$ & $\checkmark$ & $\checkmark$ & $\checkmark$ & $\checkmark$ & $\checkmark$ & $r$ & $r$ & $\checkmark$ & $r$ & & 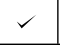 & $r$ & & & $\checkmark$ \\
\hline w.wa & $\checkmark$ & $\checkmark$ & $\checkmark$ & $\checkmark$ & $\checkmark$ & $\checkmark$ & $\checkmark$ & $\checkmark$ & $\checkmark$ & $\checkmark$ & $\checkmark$ & $\checkmark$ & & $\checkmark$ & $\checkmark$ & & & $\checkmark$ \\
\hline w.wreg & $\checkmark$ & $\checkmark$ & $\checkmark$ & $\checkmark$ & $\checkmark$ & $\checkmark$ & $\checkmark$ & $\checkmark$ & $\checkmark$ & $\checkmark$ & $\checkmark$ & $\checkmark$ & & $\checkmark$ & $\checkmark$ & & & $\checkmark$ \\
\hline w.except & $\checkmark$ & $\checkmark$ & $\checkmark$ & $\checkmark$ & $\checkmark$ & $\checkmark$ & $\checkmark$ & $\checkmark$ & $\checkmark$ & $\checkmark$ & $\checkmark$ & $\checkmark$ & & $\checkmark$ & 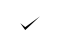 & & & $\checkmark$ \\
\hline w.twcwp & $\checkmark$ & $\checkmark$ & $\checkmark$ & $\checkmark$ & $\checkmark$ & $\checkmark$ & $\checkmark$ & $\checkmark$ & $\checkmark$ & $\checkmark$ & $\checkmark$ & $\checkmark$ & & $\checkmark$ & $\checkmark$ & & & $\checkmark$ \\
\hline
\end{tabular}


Table A.2: DE Stage IF Statistic

\begin{tabular}{c|l|r|c|l|r}
\hline \hline Index & Register & IF & Index & Register & IF \\
\hline 1 & r.d.pc & 1 & 18 & r.d.divrdy & 0 \\
2 & r.d.inst(0) & 1 & 19 & r.d.pcheld & 0 \\
3 & r.d.cwp & 1 & 20 & r.d.rexbuf & 0 \\
4 & r.d.mexc & 1 & 21 & r.d.rexcnt & 0 \\
5 & r.d.cnt & 1 & 22 & r.d.rexpl.opout & 0 \\
6 & r.d.pv & 1 & 23 & r.d.rexpl.szout & 0 \\
7 & r.d.annul & 1 & 24 & r.d.rexpl.ncntout & 0 \\
8 & r.d.step & 1 & 25 & r.d.rexpl.baddr1 & 0 \\
9 & r.d.rexen & 1 & 26 & r.d.rexpl.immexp & 0 \\
10 & r.d.rexpos & 1 & 27 & r.d.rexpl.immval & 0 \\
11 & r.d.inull & 0.976078 & 28 & r.d.rexpl.getpc & 0 \\
12 & r.d.awp & 0 & 29 & r.d.rexpl.maskpv & 0 \\
13 & r.d.aw & 0 & 30 & r.d.rexpl.illinst & 0 \\
14 & r.d.paw & 0 & 31 & r.d.rexpl.nostep & 0 \\
15 & r.d.stwin & 0 & 32 & r.d.rexpl.itovr & 0 \\
16 & r.d.cwpmax & 0 & 33 & r.d.rexpl.leave & 0 \\
17 & r.d.set & 0 & & & \\
\hline \hline
\end{tabular}

Table A.3: ACC Stage IF Statistic

\begin{tabular}{c|l|r|c|l|r}
\hline \hline Index & Register & IF & Index & Register & IF \\
\hline 1 & r.a.ctrl.pc & 1 & 23 & r.a.imm & 0.447919 \\
2 & r.a.ctrl.inst & 1 & 24 & r.a.rfa1 & 0.31907 \\
3 & r.a.ctrl.cnt & 1 & 25 & r.a.rfa2 & 0.31907 \\
4 & r.a.ctrl.rd & 1 & 26 & r.a.ctrl.tt & 0 \\
5 & r.a.ctrl.annul & 1 & 27 & r.a.ctrl.trap & 0 \\
6 & r.a.ctrl.wreg & 1 & 28 & r.a.rs1 & 0 \\
7 & r.a.ctrl.wicc & 1 & 29 & r.a.awp & 0 \\
8 & r.a.ctrl.wy & 1 & 30 & r.a.aw & 0 \\
9 & r.a.ctrl.ld & 1 & 31 & r.a.paw & 0 \\
10 & r.a.ctrl.pv & 1 & 32 & r.a.ldcheck1 & 0 \\
11 & r.a.ctrl.rett & 1 & 33 & r.a.ldcheck2 & 0 \\
12 & r.a.ctrl.itovr & 1 & 34 & r.a.ldchkra & 0 \\
13 & r.a.rsel1 & 1 & 35 & r.a.ldchkex & 0 \\
14 & r.a.rsel2 & 1 & 36 & r.a.wovf & 0 \\
15 & r.a.rfe1 & 1 & 37 & r.a.wunf & 0 \\
\hline
\end{tabular}


Appendix A. Critical Resource

Table A.3 cont'd

\begin{tabular}{c|l|r|c|l|r}
\hline Index & Register & IF & Index & Register & IF \\
\hline 16 & r.a.rfe2 & 1 & 38 & r.a.ticc & 0 \\
17 & r.a.cwp & 1 & 39 & r.a.step & 0 \\
18 & r.a.su & 1 & 40 & r.a.mulstart & 0 \\
19 & r.a.et & 1 & 41 & r.a.divstart & 0 \\
20 & r.a.jmpl & 1 & 42 & r.a.nobp & 0 \\
21 & r.a.bp & 1 & 43 & r.a.getpc & 0 \\
22 & r.a.bpimiss & 1 & 44 & r.a.decill & 0 \\
\hline \hline
\end{tabular}

Table A.4: EX Stage IF Statistic

\begin{tabular}{c|l|r|r|l|r}
\hline \hline Index & Register & IF & Index & Register & IF \\
\hline 1 & r.e.ctrl.pc & 1 & 22 & r.e.op2 & 0.810604 \\
2 & r.e.ctrl.inst & 1 & 23 & r.e.alusel & 0.791768 \\
3 & r.e.ctrl.cnt & 1 & 24 & r.e.jmpl & 0.651765 \\
4 & r.e.ctrl.rd & 1 & 25 & r.e.aluadd & 0.275166 \\
5 & r.e.ctrl.tt & 1 & 26 & r.e.aluop & 0.199829 \\
6 & r.e.ctrl.trap & 1 & 27 & r.e.ymsb & 0.189396 \\
7 & r.e.ctrl.annul & 1 & 28 & r.e.mulstep & 0.189396 \\
8 & r.e.ctrl.wreg & 1 & 29 & r.e.shcnt & 0.063764 \\
9 & r.e.ctrl.wicc & 1 & 30 & r.e.shleft & 0.034508 \\
10 & r.e.ctrl.wy & 1 & 31 & r.e.invop2 & 0 \\
11 & r.e.ctrl.ld & 1 & 32 & r.e.sari & 0 \\
12 & r.e.ctrl.pv & 1 & 33 & r.e.rd & 0 \\
13 & r.e.ctrl.rett & 1 & 34 & r.e.et & 0 \\
14 & r.e.ctrl.itovr & 1 & 35 & r.e.cwp & 0 \\
15 & r.e.op1 & 1 & 36 & r.e.awp & 0 \\
16 & r.e.alucin & 1 & 37 & r.e.aw & 0 \\
17 & r.e.ldbp1 & 1 & 38 & r.e.paw & 0 \\
18 & r.e.ldbp2 & 1 & 39 & r.e.icc & 0 \\
19 & r.e.su & 1 & 40 & r.e.mul & 0 \\
20 & r.e.mac & 1 & 41 & r.e.rfe1 & 0 \\
21 & r.e.bp & 1 & 42 & r.e.rfe2 & 0 \\
\hline \hline
\end{tabular}


Table A.5 cont'd

\begin{tabular}{l|l|l|l|l|l}
\hline Index & Register & IF & Index & Register & IF \\
\hline
\end{tabular}

Table A.5: ME Stage IF Statistic

\begin{tabular}{c|l|r|c|l|r}
\hline \hline Index & Register & IF & Index & Register & IF \\
\hline 1 & r.m.ctrl.pc & 1 & 20 & r.m.dci.read & 1 \\
2 & r.m.ctrl.inst & 1 & 21 & r.m.dci.write & 1 \\
3 & r.m.ctrl.cnt & 1 & 22 & r.m.dci.lock & 1 \\
4 & r.m.ctrl.rd & 1 & 23 & r.m.dci.dsuen & 1 \\
5 & r.m.ctrl.tt & 1 & 24 & r.m.dci.size & 1 \\
6 & r.m.ctrl.trap & 1 & 25 & r.m.dci.asi & 1 \\
7 & r.m.ctrl.annul & 1 & 26 & r.m.mac & 1 \\
8 & r.m.ctrl.wreg & 1 & 27 & r.m.su & 1 \\
9 & r.m.ctrl.wicc & 1 & 28 & r.m.casa & 0.962591 \\
10 & r.m.ctrl.wy & 1 & 29 & r.m.wcwp & 0.724834 \\
11 & r.m.ctrl.ld & 1 & 30 & r.m.werr & 0.688626 \\
12 & r.m.ctrl.pv & 1 & 31 & r.m.nalign & 0.018836 \\
13 & r.m.ctrl.rett & 1 & 32 & r.m.wawp & 0 \\
14 & r.m.ctrl.itovr & 1 & 33 & r.m.irqen & 0 \\
15 & r.m.result & 1 & 34 & r.m.irqen2 & 0 \\
16 & r.m.y & 1 & 35 & r.m.divz & 0 \\
17 & r.m.icc & 1 & 36 & r.m.mul & 0 \\
18 & r.m.dci.signed & 1 & 37 & r.m.casaz & 0 \\
19 & r.m.dci.enaddr & 1 & 38 & r.m.rexnalign & 0 \\
\hline \hline
\end{tabular}

Table A.6: X Stage IF Statistic

\begin{tabular}{c|l|r|c|l|r}
\hline \hline Index & Register & IF & Index & Register & IF \\
\hline 1 & r.x.ctrl.pc & 1 & 20 & r.x.ctrl.rett & 0 \\
2 & r.x.ctrl.rd & 1 & 21 & r.x.ctrl.itovr & 0 \\
3 & r.x.ctrl.trap & 1 & 22 & r.x.annulall & 0 \\
4 & r.x.ctrl.ld & 1 & 23 & r.x.set & 0 \\
5 & r.x.mexc & 1 & 24 & r.x.dci.signed & 0 \\
6 & r.x.nerror & 1 & 25 & r.x.dci.enaddr & 0 \\
7 & r.x.ctrl.annul & 0.826452 & 26 & r.x.dci.read & 0 \\
8 & r.x.result & 0.746304 & 27 & r.x.dci.write & 0 \\
9 & r.x.ctrl.wreg & 0.596678 & 28 & r.x.dci.lock & 0 \\
10 & r.x.intack & 0.596678 & 29 & r.x.dci.dsuen & 0 \\
11 & r.x.data(0) & 0.253696 & 30 & r.x.dci.size & 0 \\
\hline
\end{tabular}


Appendix A. Critical Resource

Table A.6 cont'd

\begin{tabular}{c|l|r|c|l|r}
\hline Index & Register & IF & Index & Register & IF \\
\hline 12 & r.x.ctrl.wicc & 0.189396 & 31 & r.x.dci.asi & 0 \\
13 & r.x.ctrl.wy & 0.189396 & 32 & r.x.laddr & 0 \\
14 & r.x.y & 0.189396 & 33 & r.x.rstate & 0 \\
15 & r.x.icc & 0.147247 & 34 & r.x.npc & 0 \\
16 & r.x.ctrl.inst & 0 & 35 & r.x.ipend & 0 \\
17 & r.x.ctrl.cnt & 0 & 36 & r.x.mac & 0 \\
18 & r.x.ctrl.tt & 0 & 37 & r.x.debug & 0 \\
19 & r.x.ctrl.pv & 0 & & & \\
\hline \hline
\end{tabular}

Table A.7: WB Stage IF Statistic

\begin{tabular}{c|l|r|c|l|r}
\hline \hline Index & Register & IF & Index & Register & IF \\
\hline 1 & r.w.s.tt & 1 & 16 & r.w.except & 0.768751 \\
2 & r.w.s.ef & 1 & 17 & r.w.twcwp & 0.768751 \\
3 & r.w.s.s & 1 & 18 & r.w.s.icc & 0.663357 \\
4 & r.w.s.et & 1 & 19 & r.w.s.pil & 0.626026 \\
5 & r.w.s.y & 1 & 20 & r.w.s.cwpmax & 0.542588 \\
6 & r.w.s.ec & 0.768751 & 21 & r.w.s.wim & 0.183764 \\
7 & r.w.s.ps & 0.768751 & 22 & r.w.s.stwin & 0.076293 \\
8 & r.w.s.asr18 & 0.768751 & 23 & r.w.s.cwp & 0 \\
9 & r.w.s.svt & 0.768751 & 24 & r.w.s.tba & 0 \\
10 & r.w.s.dwt & 0.768751 & 25 & r.w.s.rexdis & 0 \\
11 & r.w.s.dbp & 0.768751 & 26 & r.w.s.rextrap & 0 \\
12 & r.w.s.dbprepl & 0.768751 & 27 & r.w.s.aw & 0 \\
13 & r.w.result & 0.768751 & 28 & r.w.s.paw & 0 \\
14 & r.w.wa & 0.768751 & 29 & r.w.s.awp & 0 \\
15 & r.w.wreg & 0.768751 & & & \\
\hline \hline
\end{tabular}

